\title{
Acute-on-chronic liver failure: consensus recommendations of the Asian Pacific association for the study of the liver (APASL): an update
}

Shiv Kumar Sarin ${ }^{1}$ - Ashok Choudhury ${ }^{1}$ - Manoj K. Sharma ${ }^{1} \cdot$ Rakhi Maiwall $^{1} \cdot$ Mamun Al Mahtab $^{2} \cdot$ Salimur Rahman $^{2}$. Sanjiv Saigal ${ }^{3}$. Neeraj Saraf ${ }^{3}$ - A. S. Soin ${ }^{3} \cdot$ Harshad Devarbhavi $^{4}$. Dong Joon Kim ${ }^{5}$. R. K. Dhiman ${ }^{6}$.

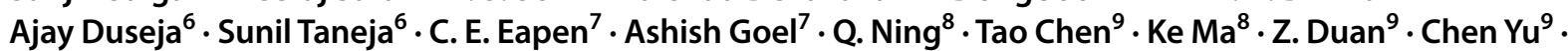
Sombat Treeprasertsuk ${ }^{10}$. S. S. Hamid ${ }^{11}$. Amna S. Butt ${ }^{11}$. Wasim Jafri ${ }^{11}$ - Akash Shukla ${ }^{12}$. Vivek Saraswat ${ }^{13}$. Soek Siam Tan ${ }^{14}$. Ajit Sood ${ }^{15}$. Vandana Midha ${ }^{15}$. Omesh Goyal ${ }^{15}$. Hasmik Ghazinyan ${ }^{16}$. Anil Arora ${ }^{17}$. Jinhua Hu ${ }^{18}$. Manoj Sahu ${ }^{19}$. P. N. Rao ${ }^{20}$. Guan H. Lee ${ }^{21}$. Seng G. Lim²1 . Laurentius A. Lesmana ${ }^{22}$. Cosmas Rinaldi Lesmana ${ }^{22}$. Samir Shah ${ }^{23}$. V. G. Mohan Prasad ${ }^{24}$. Diana A. Payawal ${ }^{25}$. Zaigham Abbas $^{26}$. A. Kadir Dokmeci ${ }^{27}$. Jose D. Sollano ${ }^{28}$. Gian Carpio $^{28}$. Ananta Shresta ${ }^{29}$. G. K. Lau ${ }^{30}$. Md. Fazal Karim ${ }^{31}$. Gamal Shiha ${ }^{32}$. Rino Gani ${ }^{33} \cdot$ Kemal Fariz Kalista $^{33}$. Man-Fung Yuen ${ }^{34}$. Seema Alam ${ }^{35} \cdot$ Rajeev Khanna $^{35} \cdot$ Vikrant Sood $^{35} \cdot$ Bikrant Bihari Lal $^{35}$. Viniyendra Pamecha ${ }^{36}$.

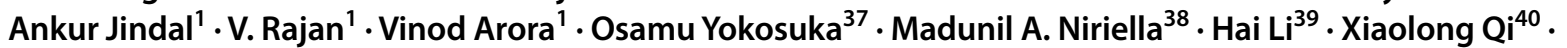
Atsushi Tanaka ${ }^{41}$ - Satoshi Mochida ${ }^{42} \cdot$ Dominic Ray Chaudhuri ${ }^{43} \cdot$ Ed Gane $^{43} \cdot$ Khin Maung Win $^{44} \cdot$ Wei Ting Chen $^{45}$. Mohd. Rela ${ }^{46}$. Dharmesh Kapoor ${ }^{23} \cdot$ Amit Rastogi $^{3} \cdot$ Pratibha Kale $^{47} \cdot$ Archana Rastogi $^{48}$. Chhagan Bihari Sharma ${ }^{48}$. Meenu Bajpai ${ }^{49} \cdot$ Virender Singh $^{6} \cdot$ Madhumita Premkumar $^{6} \cdot$ Sudhir Maharashi $^{50} \cdot$ A. Olithselvan $^{51}$. Cyriac Abby Philips ${ }^{52}$. Anshu Srivastava ${ }^{53}$. Surender K. Yachha ${ }^{53} \cdot$ Zeeshan Ahmad Wani $^{54} \cdot$ B. R. Thapa ${ }^{55}$. Anoop Saraya ${ }^{56} \cdot$ Shalimar $^{56} \cdot$ Ashish Kumar $^{17} \cdot$ Manav Wadhawan $^{57} \cdot$ Subash Gupta $^{58} \cdot$ Kaushal Madan $^{59}$. Puja Sakhuja ${ }^{60}$. Vivek Vij ${ }^{61}$. Barjesh C. Sharma ${ }^{62}$. Hitendra Garg ${ }^{63}$. Vishal Garg ${ }^{63}$. Chetan Kalal ${ }^{64}$. Lovkesh Anand $^{65}$. Tanmay Vyas ${ }^{66}$. Rajan P. Mathur ${ }^{67}$. Guresh Kumar ${ }^{68}$. Priyanka Jain ${ }^{68}$. Samba Siva Rao Pasupuleti ${ }^{68}$.

Yogesh K. Chawla ${ }^{69} \cdot$ Abhijit Chowdhury $^{70}$. Shahinul Alam ${ }^{2}$. Do Seon Song ${ }^{71}$ - Jin Mo Yang ${ }^{71}$ - Eileen L. Yoon ${ }^{72}$. APASL ACLF Research Consortium (AARC) for APASL ACLF working Party.

Received: 1 January 2019 / Accepted: 3 April 2019 / Published online: 6 June 2019

(c) The Author(s) 2019, corrected publication 2019

\begin{abstract}
The first consensus report of the working party of the Asian Pacific Association for the Study of the Liver (APASL) set up in 2004 on acute-on-chronic liver failure (ACLF) was published in 2009. With international groups volunteering to join, the "APASL ACLF Research Consortium (AARC)" was formed in 2012, which continued to collect prospective ACLF patient data. Based on the prospective data analysis of nearly 1400 patients, the AARC consensus was published in 2014. In the past nearly four-and-a-half years, the AARC database has been enriched to about 5200 cases by major hepatology centers across Asia. The data published during the interim period were carefully analyzed and areas of contention and new developments in the field of ACLF were prioritized in a systematic manner. The AARC database was also approached for answering some of the issues where published data were limited, such as liver failure grading, its impact on the 'Golden Therapeutic Window', extrahepatic organ dysfunction and failure, development of sepsis, distinctive features of acute decompensation from ACLF and pediatric ACLF and the issues were analyzed. These initiatives concluded in a two-day meeting in October 2018 at New Delhi with finalization of the new AARC consensus. Only those statements, which were based on evidence using the Grade System and were unanimously recommended, were accepted. Finalized statements were again circulated to all the experts and subsequently presented at the AARC investigators meeting at the AASLD in November 2018. The suggestions from the experts were used to revise and finalize the consensus. After detailed deliberations and data analysis, the original definition of ACLF was found to withstand the test of time and be able to identify a homogenous group of patients presenting with liver failure. New management options including the algorithms for the management of coagulation disorders, renal replacement
\end{abstract}

Extended author information available on the last page of the article 
therapy, sepsis, variceal bleed, antivirals and criteria for liver transplantation for ACLF patients were proposed. The final consensus statements along with the relevant background information and areas requiring future studies are presented here.

\section{Article Highlights}

- Updated on the basis of AARC data of $>3300$ cases enrolled into AARC registry prospectively

- ACLF is distinct form Acute Decompensation of cirrhosis

- Newer sections on DILI-ACLF, AIH-ACLF, PVT/HVOTO-ACLF

- Reversibility of Chronic Liver Disease in ACLF

- Portal and systemic hemodynamics and their relevance in ACLF

- Acute Portal Hypertension and Variceal progression in ACLF

- AARC score as a guide for treatment strategies in ACLF

- ACLF in Children-first consensus on pediatric ACLF

Keywords Liver failure - Cirrhosis · Jaundice - AARC - Chronic liver disease - Alcoholic liver disease - ALF . Decompensation $\cdot$ Acute decompensation

$\begin{array}{ll}\text { Abbreviations } & \\ \text { ACLF } & \begin{array}{l}\text { Acute-on-chronic liver failure } \\ \text { drug-induced liver injury } \\ \text { DILI }\end{array} \\ \text { CAM } & \begin{array}{l}\text { Complimentary and alternative } \\ \text { medicine }\end{array} \\ \text { HDS } & \text { Herbs, drugs and supplements } \\ \text { AD } & \text { Acute decompensation } \\ \text { AIH } & \text { Auto immune hepatitis } \\ \text { BCS } & \text { Budd-Chiari syndrome } \\ \text { PVT } & \text { Portal vein thrombosis } \\ \text { AVB } & \text { Acute variceal bleed } \\ \text { PICD } & \text { Paracentesis induced circulatory } \\ & \text { dysfunction } \\ \text { RRT } & \text { Renal replacement therapy } \\ \text { LT } & \text { Liver transplant } \\ \text { SOFA } & \text { Sequential organ failure assessment } \\ \text { qSOFA } & \text { Quick sequential organ failure } \\ \text { CANONIC-CLIF } & \text { Assessment } \\ & \text { Acute-oN-ChrONic LIver Failure in } \\ \text { CLIF } & \text { Cirrosis } \\ \text { MELD } & \text { Model end-stage liver disease } \\ \text { TPPM } & \text { Tongji prognostic predictor model }\end{array}$

\section{Introduction}

Liver failure is a common medical ailment and its incidence is increasing with the use of alcohol and growing epidemic of obesity and diabetes. It can present as acute liver failure (ALF) (in the absence of any pre-existing liver disease), acute-on chronic liver failure (ACLF) (an acute deterioration of known or unknown chronic liver disease), or an acute decompensation of an end-stage liver disease [1, 2]. Each of these is a well-defined disease entity with a homogenous population of patients with expected outcomes. Due to an overlap and lack of clarity of definitions and outcomes, entities like late-onset liver failure, sub-acute hepatic failure, have become less relevant and there is lack of further publications suggesting removal of such terminologies to avoid confusion $[1,2]$.

The growing interest in ACLF after the first consensus definition of ACLF from APASL [2] is evident by the fact that more than $>450$ publications as full papers have been published from the West (2) and the East and the trend is increasing. The group of investigators working on liver failure in the Asia-Pacific region working for the past decade carefully analyzed the patient characteristics, natural history and outcome of such patients. The group met on yearly basis and collated data on website (www.aclf.in). With the setting up of the APASL ACLF Research Consortium (AARC) in 2012, the collaborative research work, publications and protocol driven unified treatment had gained momentum. The retrospective and prospective data of patients from different centers were analyzed, and the completed patient records were utilized for defining predictors of mortality and grades of liver failure and incidence of other organ failures [3].

The APASL ACLF consensus of 2014 was based on about 1363 patients from 14 countries. During the past nearly four and a half years (2014-2018), 5228 patients of 43 Centers from 15 countries have so far been registered in the AARC database. These patients have been prospectively enrolled and followed and form the basis of the new structured consensus.

Experts from across the world, especially from the Asia-Pacific region, were requested to identify pertinent and contentious issues in ACLF. After a round of deliberations, 8 major issues were identified for update. Further, data from the AARC database were taken and analyzed and circulated to all the participants.

The process for the development of the recommendations and guidelines included: review of all available published 
literature on ACLF by individual and group of experts; preparation of a review manuscript and consensus statements based on GRADE SYSTEM (Table 1) of evidencebased approach [4], circulation of consensus statements to all experts, a survey of the current approaches for the diagnosis and management of ACLF; discussion on contentious issues; and deliberations to prepare the consensus statement by the experts of the working party. A 2-day meeting was held on October 1-2, 2018, at New Delhi, India, to discuss and finalize the consensus statements, recommendations and guidelines. The finalized statements were circulated to all the experts and subsequently finalized. These consensus statements and recommendations for the diagnosis and management of ACLF are included in this review. A brief background is included providing the available data and published information on each of the issues. Statements from the previous consensus have been reproduced at places to give a background and continuity.

\section{The concept of ACLF and hepatic reserve}

Acute liver failure is a well-defined medical emergency which is defined as a severe liver injury, leading to coagulation abnormality usually with an INR $\geq 1.5$, and any degree of mental alteration (encephalopathy) in a patient without pre-existing liver disease and with an illness of up to 4 weeks duration [5].A proportion of patients who present with features mimicking ALF, however, have an underlying chronic liver disease or cirrhosis of the liver. These patients grouped together as acute-on-chronic liver failure (ACLF) also have a poor outcome. These patients are distinctly different from a group of cirrhotic patients who are already decompensated [6] and have a sudden worsening of their condition, i.e., acute decompensation (AD) due to an acute event that may present with hepatic or non-hepatic failure [6].

ACLF is a clinical syndrome manifesting as acute and severe hepatic derangements resulting from varied insults. This term was first used in 1995 to describe a condition in which two insults to liver operate simultaneously, one of them being ongoing and chronic and the other acute [7]. Over the years, several definitions have been proposed, creating confusion in the field [8]. The time frame for the development of liver failure and ACLF has been several times changed from 12 to 4 weeks again to 12 weeks [9]. Moreover, the nature of insult and the stage of underlying disease have been variably used.

In fact, any patient who has an underlying chronic liver disease with superimposed acute insult is labeled as having ACLF, irrespective of evidence of liver failure per se or evidence of pre-existing cirrhotic decompensation. Several investigators were concerned that this would lead to substantial overlap with decompensated liver disease. The main emphasis of the fourth consensus meeting of the APASL Working Party was to identify from this large group of patients, a subset of patients who have a relatively homogenous presentation and potentially similar outcome and restrict the use of the term "acute-on-chronic liver failure" to this subset. The 2009 APASL definition had provided a basis to select patients presenting with a distinct syndrome. To cover the entire spectrum of these patients, from mild to most severe, patients with chronic liver disease with or without cirrhosis of the liver were included and carefully analyzed. It is understandable, though not well defined, that the nature and degree of acute insult and the status of the underlying chronic liver disease would determine the outcome in a patient (Fig. 1).

To give clarity to the primary event, a hepatic insult, jaundice and coagulopathy, which defined liver failure, was considered essential. In acute liver failure, though hepatic encephalopathy (HE) is part of the definition, it follows liver failure. Should one wait for defining the outcome of 'acute liver failure' till the time extrahepatic organ failures set in or not, remains contentious. For definition, the event must be universally present in all patients. From the point of view of intensivists, it is well known that with increasing number of organ dysfunction or failure, the mortality would cumulatively increase. Undoubtedly, these events are predictive of the outcome, the basis of SOFA score [10]. It is, therefore,

Table 1 Evidence grade used for the APASL Guidelines Adopted from Atkins et al. [4]

\begin{tabular}{|c|c|c|}
\hline Grading of evidence & Notes & Symbol \\
\hline High quality & Further research is very unlikely to change our confidence in the estimate effect & A \\
\hline Moderate quality & $\begin{array}{l}\text { Further research is likely to have an important impact on our confidence in the estimate of effect } \\
\text { and may change the estimate effect }\end{array}$ & B \\
\hline Low or very low quality & $\begin{array}{l}\text { Further research is very likely to have an important impact on our confidence in the estimate of } \\
\text { effect and may change the estimate effect. Any estimate of effect is uncertain }\end{array}$ & $\mathrm{C}$ \\
\hline Grading of recommendations & Notes & Symbol \\
\hline Strong recommendation warranted & $\begin{array}{l}\text { Factors influencing the strength of the recommendation included the quality of the evidence, } \\
\text { presumed patient-important outcomes, and cost }\end{array}$ & 1 \\
\hline Weaker recommendation & $\begin{array}{l}\text { Variability in preferences and values, or more uncertainty: more likely a weak recommendation is } \\
\text { warranted. Recommendation is made with less certainty: higher cost or resource consumption }\end{array}$ & 2 \\
\hline
\end{tabular}


Fig. 1 Concept of ACLF and the cohorts included in different definitions. The figure describes the response of the liver to an acute hepatic injury, depending on the underlying hepatic injury, prior decompensation, time frame from insult to presentation with decompensation and reversibility with mitigation of the acute insult. The spectrum extends from acute liver failure, acute-on-chronic liver failure, acute decompensation, end-stage liver disease. ACLF is distinct like ALF when the APASL definition is considered. APASL definition is simple and homogenous and is distinct

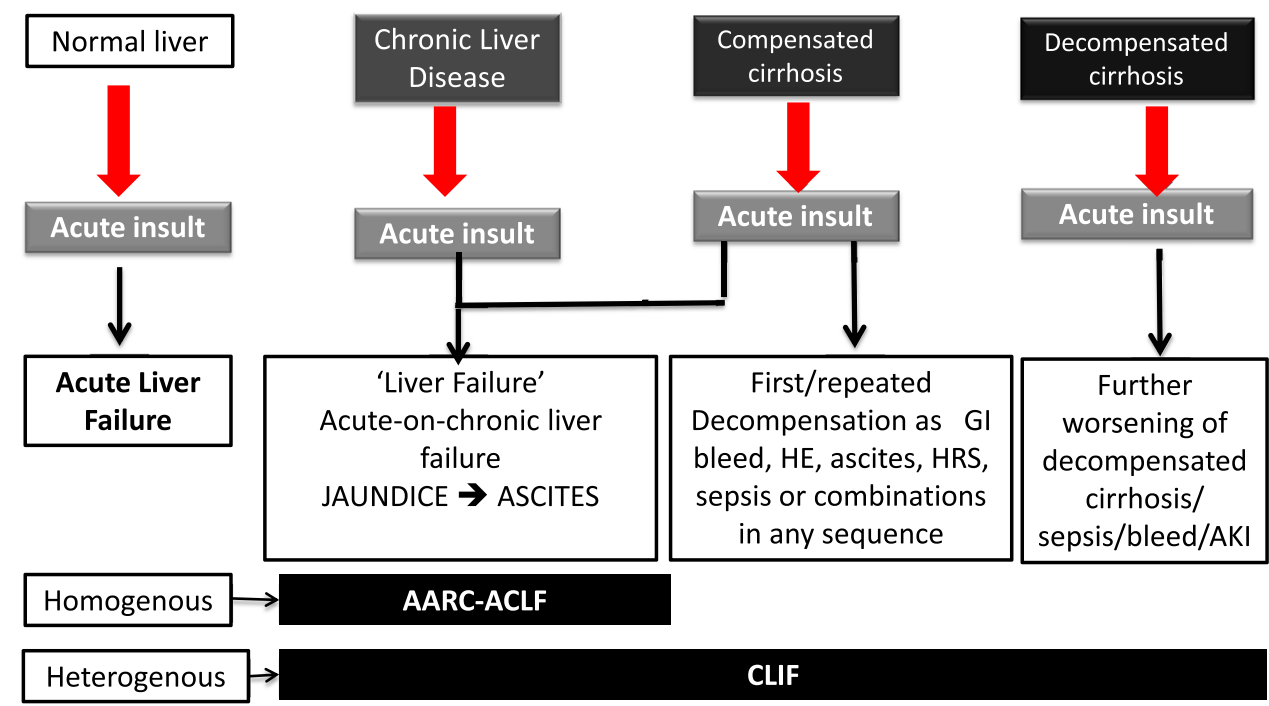

not surprising; the same has been reported in the Western studies [11]. However, should organ failure be included in defining the clinical syndrome of liver failure needs a thorough analysis. As a corollary, despite decades of extensive experience, renal or circulatory dysfunction has not been included in the definition of ALF. The issue whether sepsis per se could lead to liver failure or is a result of liver failure had been debated for many years and was again revisited. However, sepsis is an integral part of development of multiorgan failure in any patient, be it of renal, pancreatic or cardiac origin.

In essence, ACLF is a distinct entity where acute hepatic decompensation occurs in an established chronic liver disease or cirrhosis patient on exposure to acute insult in a defined time frame resulting in a high short-term mortality. Based on the data, attempts were made if the current definition of ACLF could be improved further (Table 2). Five aspects were worked upon:

(1) The time frame for the acute insult in the initial (2009) and subsequent definition of ACLF, the time for development of ascites and/HE after appearance of jaundice and coagulopathy was kept as 4 weeks (28 days) $[1,2]$. A mortality rate of more than $33 \%$ at 4 weeks was considered to be significant allowing recovery to less than two-third of the patients in the 2014 consensus. The additional new data after the previous consensus were carefully analyzed and it showed a 4-week mortality of around 39.9\% [2]. Therefore, the definition of 4 week for acute insult in ACLF was considered as appropriate and was maintained.

2. Reversibility of the ACLF syndrome this is a feature of the ACLF defined by the AARC criteria, as nearly all the patients included are after the index presentation. With mitigation of acute insult and over time, the hepatic reserve improves, fibrosis regresses and the portal pressure decreases. It was decided to define reversibility as reversal of key components that were used for defining the syndrome of liver failure, i.e., decrease of bilirubin below $5 \mathrm{mg} / \mathrm{dL}$ and reversal of coagulopathy to INR below 1.5 and no encephalopathy with or without resolution of ascites. It was interesting to find in the large AARC database, of the 1844 patients with complete data until day 90 . About $70 \%$ of the survivors beyond day 90 , showed reversibility and they maintained this status for a period of at least 1 year. Further, unlike patients with decompensated cirrhosis and similar to patients with ALF, the reversal of coagulopathy preceded the reversal of jaundice, i.e., median time to reversal of coagulopathy was 7 (4-30) days versus 19 (7-60) days for jaundice, respectively. The median time to reversal of syndrome, i.e., jaundice and coagulopathy, was 30 days. Baseline albumin, AARC score and transient elastography predicted long-term reversibility in the recently analyzed AARC data.

3. Recording 'Index 'or first presentation in the definition of ACLF this issue was deliberated so as to define and include a homogenous cohort of patients. The consideration of prior decompensation with recent worsening (difficult to differentiate from acute decompensation, AD) or recovery from ACLF and followed by subsequent presentation as ACLF (i.e., 'ACLF again') will lead to confusion. It is important to distinguish the syndrome of ACLF from other forms of liver failure, such as acute decompensation and end-stage liver disease (ESLD). There was a consensus to initiate prospective studies comparing patient manifesting with index presentation, prior decompensation or recent worsening of decompensated cirrhosis patients. 


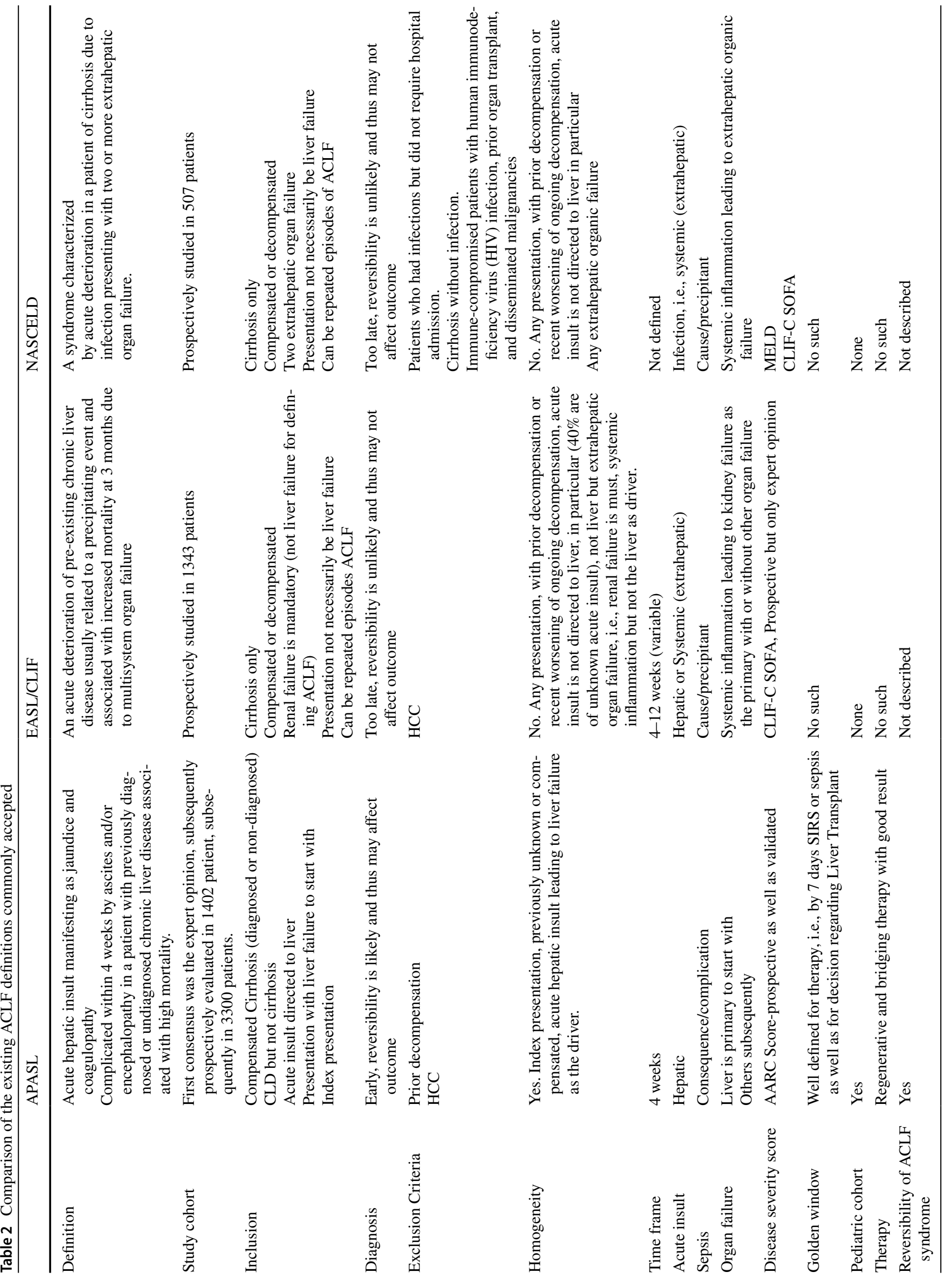


4. Inclusion of mortality in definition the mortality was included in 2014 AARC consensus definition for identifying a set of patients who have high 28-day mortality so as to prioritize them for admission, treatment and liver transplantation. At present, the ACLF definition both by the APASL and CLIF-EASL includes mortality. The group of experts raised the concern that mortality is generally not part of definition in disease conditions. Other experts, however, disagreed to this. After due deliberations, it was decided to keep the statement on mortality, in the AARC-ACLF definition.

5. Inclusion of organ failure in definition the Western definitions of ACLF include organ failure in the definition. This issue was debated extensively. The data from the AARC database were also analyzed. It was reiterated that organ failure other than liver should not be part of the definition. Diagnosis of liver failure along with kidney, circulatory and respiratory failure is generally a late event and is often a result of the primary organ, i.e., liver failure (jaundice, deranged coagulation and/or HE). The experts felt that organ dysfunction rather than organ failure should be the time for raising suspicion and making diagnosis of ALCF rather than when organ failure(s) has already developed. This approach would help in prioritizing organ-specific interventions.

The AARC definition of ACLF is a simple bed-side tool (requires history taking, physical examination and simple laboratory parameters) and can be used by primary care givers. It enables a clinician to stratify a patient presenting with liver failure for early referral, early intervention and, hence, allows a better chance of reversibility as well as improved survival. The earlier criteria for defining the nature of acute insult were reiterated, i.e., the event must be new and acute and its impact on the patient's condition should be discernible as liver failure within a given time frame of 4 weeks $[1,2]$.

\section{Recommendations}

1.0 The concept of ACLF and hepatic reserve.

1.1 The 28- and 90-day mortality is high in ACLF patients (A1).

1.2. Among the survivors at day 90, the reversal of ACLF syndrome was noted in nearly $70 \%$ cases (C2).

1.3. Almost two-third of the patients, who had reversal of the ACLF syndrome by day 90 , show a persistent regression of the disease at 1 year $(\mathrm{C} 2)$.

1.4. Reversal of coagulopathy precedes the reversal of jaundice (C2).

1.5. The baseline AARC liver failure grade can identify patients who are likely to reverse $(\mathrm{C} 2)$.
1.6. A higher platelet count, lower leukocyte count and the absence of $\mathrm{HE}$ are additional independent predictors of reversibility (C2).

1.7. Transient elastography needs to be evaluated for identifying the reversibility of ACLF syndrome at baseline as well as at follow-up (C2).

$1.8 \quad$ Will inclusion of terminology of 'First' presentation in definition improve clarity and homogeneity.

1.8.1. Inclusion or exclusion of prior decompensation and 'first' presentation for the definition of ACLF needs prospective studies [B2].

1.9. Including organ failure in definition-for utility or futility?

1.9.1. The terms "organ dysfunction" and "organ failure" need to be described more clearly based on the AARC database, used in APASL consensus [B2].

1.9.2. Extrahepatic organ failure should not be included in definition of ACLF, as this would lead to missing out the potential therapeutic window for reversal of the ACLF syndrome (A1).

1.9.3. Liver failure for definition of ACLF should include jaundice (serum bilirubin $\geq 5 \mathrm{mg} / \mathrm{dL}$ ) and coagulation dysfunction (INR $\geq 1.5$ ) (A1).

1.10. Whether mortality should be part of definition?

1.10.1. Mortality should not be part of the definition of ACLF. One need not die to fulfill the criteria of ACLF definition. Mortality is not generally part of any definition in disease conditions (C2). However, since the earlier (2014) definition had included mortality, the same definition was agreed.

\section{Definition of ACLF}

There is no consistent definition of ACLF in the literature. Each study done previously on ACLF has used its own definition, and there is no unanimity in these definitions in terms of criteria for liver failure, the acute event precipitating ACLF, and the diagnosis of underlying chronic liver disease. Since most of these studies were on patients who required liver support devices or liver transplantation, these studies were biased toward including sicker patients in the definition and patients having a mild disease were left out.

A detailed analysis of the definition of liver failure and the need for the defined outcome of high 28-day mortality was taken into account. An estimated $33 \%$ mortality at 28 days was considered important. Having analyzed and defined the acute and chronic insults, the time frame and the criteria of liver failure, development and course of organ failure and sepsis, the APASL definition of ACLF of 2009 was reassessed. It was reported that this definition has been used in nearly 200 publications from the East and the West and has been found to be simple to use and with a high degree of predictive ability to define the outcome of a 
relatively homogenous group of liver failure patients with underlying chronic liver disease.

The consensus definition is:

"ACLF is an acute hepatic insult manifesting as jaundice (serum bilirubin $\geq 5 \mathrm{mg} / \mathrm{dL}$ ( $85 \mathrm{micromol} / \mathrm{L}$ ) and coagulopathy (INR $\geq 1.5$ or prothrombin activity $<40 \%$ ) complicated within 4 weeks by clinical ascites and/or encephalopathy in a patient with previously diagnosed or undiagnosed chronic liver disease/cirrhosis, and is associated with a high 28-day mortality." (I, A).

\section{Defining the acute insult}

The spectrum of acute insult in the Asian region was revisited, while hepatitis B reactivation remains the predominant cause of acute hepatic insult in the East, from the global perspective. The trends showed an increase in the proportion of alcoholic hepatitis over the years. This was a bit unexpected for the Asian countries where alcoholic hepatitis is emerging as a major acute insult and shows the growing westernization of Asia. A review of the recent CANONIC study data showed that in the West the term precipitating event is generally used and probably details of events such as Hepatitis B or superadded hepatitis A and E are rarely encountered or recorded [11]. Surprisingly, even the active alcohol abuse and alcoholic hepatitis were also not the predominant causes. A plausible explanation could be that since the CANONIC study only recorded the acute decompensation of cirrhosis and not the hepatic insults, the major events recorded were only non-hepatic, such as bacterial infections or sepsis. Acute decompensation of cirrhosis is a different entity than ACLF. As the core premise of ACLF is presentation as liver failure, the acute insults should be hepatic insults. Both hepatotropic and non-hepatotropic insults should manifest in the patient first with liver failure.

Acute hepatic insults of infectious etiology included reactivation of hepatitis B virus (HBV) as the leading cause of ACLF in the Asian region [12-20]. Reactivation of HBV could be either spontaneous or due to intensive chemotherapy or immunosuppressive therapy $[12,13]$, immune restoration after highly active antiretroviral therapy for HIV [14, 15], treatment related [16], or reactivation of the occult HBV infection by rituximab (anti-CD20)-based chemotherapy [17-19]. Similarly, reactivation of hepatitis $C$ virus infection has also been reported, especially after immune suppressive therapy $[20,21]$. The other very important infectious etiology of the acute event is super-infection with hepatitis E virus, predominantly in patients in the Indian subcontinent [22-25]. Various bacterial, parasitic, and fungal infections may affect the liver. Spirochetal, protozoal, helminthic, or fungal organisms may directly infect the liver, whereas bacterial or parasitic infection may spread to the liver from other sites [26]. These infections may lead to liver failure in patients with underlying chronic liver disease. Among the non-infectious etiologies, alcoholic hepatitis is the major cause of acute deterioration in stable known or unknown chronic liver diseases, more often in the western countries $[27,28]$. It was not clear what should be the interval from the last alcoholic drink to be included as acute insult. Since, after the direct hepatic injury, the immunological injury starts to decline [29], a period of 28-day was considered adequate for inclusion as the last drink. The issue, which remained to be addressed, was of binge drinking in patients with ACLF due to recent alcohol intake. It was appreciated that a prospective data collection including the drinking behavior especially in the past 6 months would help decide the influence of drinking behavior on the clinical outcome and help in defining the time frame of what should be considered as an acute insult (Fig. 2).

(A) Drug-induced liver injury (DILI) presenting as ACLF is an important entity less often addressed in the Global literature. Hepatotoxic drugs and complementary and alternative medicines (CAM) are important causes for acute and acute-on-chronic liver failure in the Asia-Pacific region [30]. While most drugs are safely tolerated in the setting of CLD, recent work suggests that individuals with CLD may be at increased risk to develop hepatotoxicity at least to certain drugs [31]. Hepatitis following the use of anti-tubercular drugs was considered to be an important cause of acute insult leading to ACLF. In a proportion of patients, despite a history of use of CAM, the precise nature and injurious influence of the agent cannot be determined. Results from the drug-induced liver injury network have demonstrated that mortality in 89 patients with pre-existing liver disease was $16 \%$ which was significantly higher than the $5 \%$ mortality in 810 patients with underlying liver disease [32]. Drugs such as antituberculosis drugs, methotrexate and antiretroviral drugs in HIV/AIDS-infected individuals have been implicated as triggering liver injury include particularly in the setting of underlying chronic liver disease due to hepatitis B or C [33-37]. Paradoxically CLD or cirrhosis is a risk factor for tuberculosis $[38,39]$ and first-line anti-tuberculosis drugs have been consistently shown to increase the risk of hepatotoxicity, particularly in hepatitis B and C [40, 41]. Although drugs have been listed as a precipitant factor in ACLF, data are scarce except from the APASL/AARC database. Data from the West is lacking on drugs as an acute insult leading to ACLF. In Asia 1.8\% [42] to 5.7\% $[43,44]$ precipitating events for ACLF are related to drugs. In Chinese cohort, the drugs were mostly from herbal or traditional medications to anti tuberculosis drugs in Indian cohorts [44]. From the AARC database, 329 (10\%) of the 3132 patients of ACLF had an inciting event due to drugs. There is, however, need for further data and work on the 
modes of hepatic injury caused by different herbal and medicinal preparations on patients with cirrhosis.

(B) Autoimmune hepatitis flare leading to ACLF has not been adequately addressed in both Asia Pacific as well as Western cohorts. The pattern of clinical presentation spans from benign chronic hepatitis and indolent disease to acute liver failure. The abrupt presentation can indicate spontaneous exacerbation of a pre-existent chronic disease (presenting as ACLF), newly developed disease (presenting as ALF), a superimposed infectious or toxic injury, or new disease after viral infection, drug therapy, or liver transplantation. Approximately, 20\% of patients with autoimmune hepatitis present with severe jaundice, HE and coagulopathy, with or without ascites, which resemble ALF or ACLF [45]. The disease usually has an unusual presentation with nearly half the patients being seronegative, requiring a lower threshold for transjugular liver biopsy. The histological features are distinct from those found in fulminant AIH. Stravitz et al. [46] identified lymphoid aggregates, perivenulitis and massive hepatitic necrosis as suggestive histological features of AIH-ALF [47]. The multicentric AIH-ACLF data from AARC database, which showed that the lymphoid aggregates and perivenulitis are less common in AIH-ACLF. However, advanced fibrosis (F3/F4), ductular reactions, and large areas of parenchymal collapse with lymphoplasmacytic inflammation are prominent findings along with classical autoimmune features in AIH-ACLF. It was observed that use of steroid in a select group of moderately severe $\mathrm{AIH}$ has a favorable outcome. Autoimmune hepatitis (AIH) was the first liver disease for which an effective therapeutic intervention was provided and treatment efficacy shown [47].

(C) Acute variceal bleeding has been included as one of the events to define acute decompensation in the natural history of cirrhosis [48]. Variceal bleeding has also been taken as an acute insult for ACLF in some western trials of ACLF. A scenario may exist that a patient who has already fulfilled the criteria of ACLF and has been diagnosed ACLF, develops a variceal bleed. In such a patient, variceal bleed would be considered as a complication in the natural history of ACLF. In recently analyzed 1028 compensated cirrhosis patients presenting with acute variceal bleed, the syndrome of ACLF was seen in $4 \%$ cases. Acute variceal bleed led to $10 \%$ mortality in compensated cirrhosis, which increased to $18 \%$ in 90-day follow-up with the development of ACLF $(p<0.001)$. A large set of data was mined and the issue was debated whether to consider variceal bleed as an acute event of ACLF. However, since the definition of ACLF includes liver failure, jaundice and coagulopathy, the variceal bleed should result in liver failure. The liver failure in such patients is likely to be due to hepatic ischemia [49] and subsequent bacterial infections [50]. It was discussed that for a patient with portal hypertension and cirrhosis of the liver who presents for the first time with variceal bleed without any previous or present signs or symptoms of

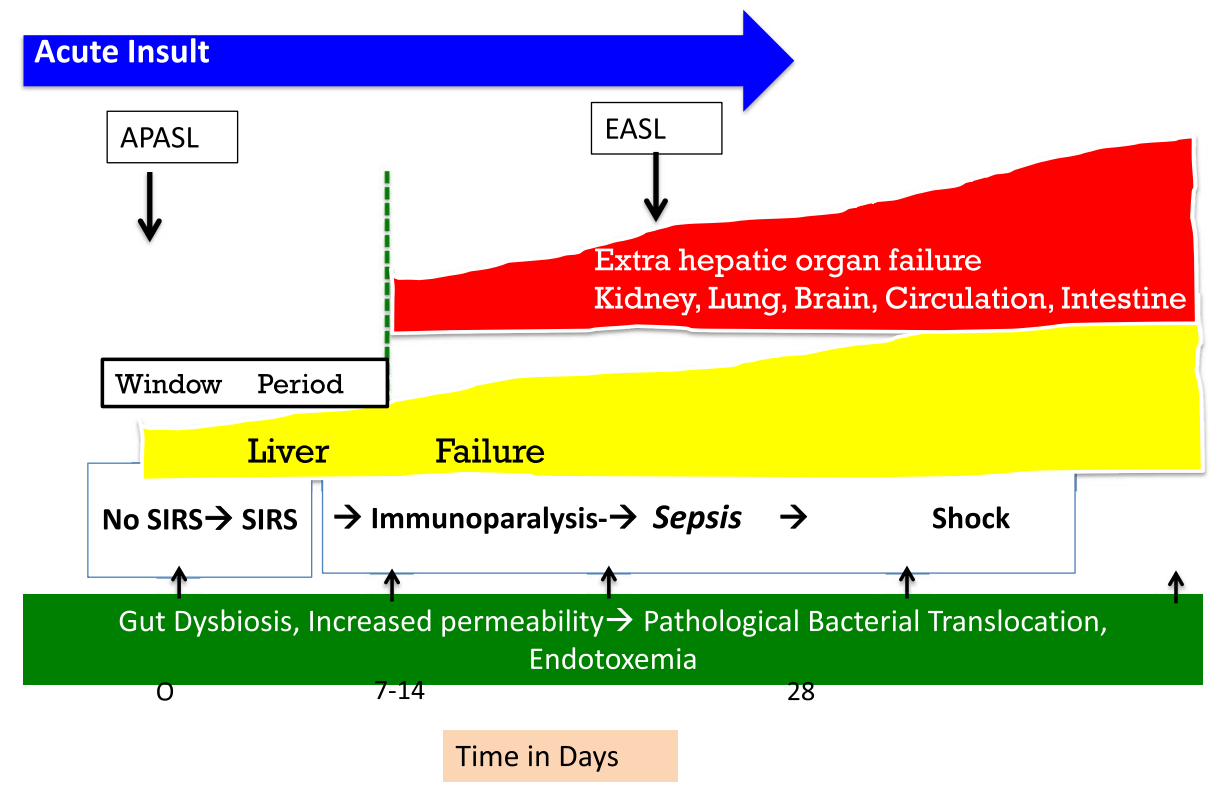

Fig. 2 Sequence of events in Diagnostic Criteria of ACLF: East vs. West. The figure clearly describes the sequence of organ failure and its mechanism. An acute hepatic insult leading to hepatic decompensation is the driver and subsequent extrahepatic organ failure is due to failure of recovery/regeneration and development of sepsis after a Golden Window. With consideration of sepsis as the intiating factor and development of extrahepatic organ involvement as a part of defi- nition leads to late identification of the ACLF patients where the therapeutic windos is lost. The difference between ACLF, AD and ESLD (as in Fig. 1) is blurred and entity is heterogenous. So pure hepatic insult leading to hepatic failure at the beginning and subsequent extrahepatic organ failure as complication, not defining complex is the crux in managing this group of liver disease patient 
chronic liver disease, it would not constitute an acute insult. This is especially relevant if such a patient does not develop any jaundice. The experts discussed the stratification of patients based on the stage of underlying liver disease and the severity of variceal bleed. Based on the data, it was unanimously agreed that acute variceal bleeding is not an acute hepatic insult unless in the patients where it produces jaundice and coagulopathy fulfilling the criteria of ACLF.

(D) Acute HVOTO or PVT presenting as ACLF is a novel concept. The disease burden, clinical picture, prognosis and treatment strategies of BCS or PVT presenting as ACLF are largely unknown. The thrombophilic disorders in those with ACLF have not been evaluated but are unlikely to be different from those without ACLF. The reduction of hepatic blood flow due to acute PVT may lead to ischemic liver injury [51]. The diagnosis of acute-on-chronic BCS in the study by Langlet et al. was based on the presence of both acute and chronic features, clinically and/or radiologically [52]. However, the entity of ACLF was not described at that time and it is unclear if any of these patients would have fulfilled the criteria of ACLF. However, it was reported that these patients had worse outcome as compared to other patients with Budd-Chiari syndrome. Evaluation of thrombophilic disorders in patients with PVT or BCS and ACLF should be similar to those without ACLF. There is no evidence currently to suggest that non-cirrhotic portal fibrosis or EHPVO may present as ACLF.

The issue of other non-hepatotropic insults which have been considered in other studies such as surgery, trauma, insertion of transjugular intrahepatic portosystemic shunt, trans-arterial chemoembolization or radiofrequency ablation for hepatocellular carcinoma was discussed in detail. While there is an indirect connection with each of these, it was debated that a patient who already has cirrhosis with HCC or a cirrhotic who undergoes surgery, separate risk scores are already in practice and being utilized. The likely potential for hepatic decompensation would vary depending on the nature of intervention and underlying hepatic reserve. It was agreed that non-hepatotropic insults producing direct hepatic insult and ACLF in an otherwise compensated liver disease could be considered as acute hepatic insults. In a proportion of patients in Asia or even in the west, the precise agent(s) leading to acute hepatic insult are not well recognized on routine assessment. In such patients, this should be recorded as such.

\section{Recommendations}

\subsection{Defining the acute event in ACLF.}

2.1.1. Infections.

2.1.1. Hepatotropic infections.

2.1.1.1. Hepatotropic viral infections: In this group, reactivation of Hepatitis B virus (HBV) infection and super-infection with hepatitis virus are the major causes of acute insults for precipitating $\operatorname{ACLF}(1, \mathrm{~A})$.

2.1.1.2 Hepatotropic non-viral infections: These include bacterial, parasitic, and fungal infections precipitating liver failure and $\operatorname{ACLF}(2, \mathrm{C})$.

2.1.2. Non-hepatotropic infections.

2.1.2.1. Bacterial infection is unlikely to be the precipitant in individuals with a definite hepatotropic acute insult (2, B).

2.1.2.2. Bacterial infections, if they primarily precipitate hepatic failure, and present as ACLF, may be considered as a precipitant of ACLF, but data at present are inadequate to demonstrate that infection per se could lead to jaundice and liver failure $(2, \mathrm{C})$. Druginduced liver injury (Drugs, CAM \& HDS)—with or without cirrhosis.

2.1.2.1. Drug-induced ACLF (ACLF-D) is a distinct entity than DILI [1, A].

2.1.2.2. Diagnosis of ACLF-D is challenging as liver disease-related fluctuations in the liver function tests may be part of the natural history of the disease and may confound the diagnosis. Further, cirrhotic patients may not show marked derangements in transaminases [1, B].

2.1.2.3. Those who develop ACLF-D are likely to have severe consequences including decompensation and death $(1, \mathrm{~B})$.

2.1.2.4. Drugs responsible as acute insults, triggering ACLF-D in cirrhosis patients include antitubercular drugs, Complimentary and alternative medications, antiretroviral drugs and Methotrexate $(1, \mathrm{~B})$. More evidence is needed for drugs like azithromycin, azole antifungals and antimicrobials in cirrhotics $(2, \mathrm{~B})$.

2.1.2.5. Risk of liver injury is proportional to the number of hepatotoxic drugs in anti-TB regimen $(2, \mathrm{C})$.

2.1.3. Autoimmune liver disease - distinction in presentation as $A C L F$ and ALF.

2.1.3.1. ACLF-AIH frequently presents as seronegative for autoantibodies or normal IgG levels [B2]. Seronegative AIH cases might be overlooked without histology [1, B].

2.1.3.2. Diagnosis of ACLF-AIH requires liver biopsy (transjugular route preferred). Biopsy is more helpful in patients where etiology is not evident; antibodies and $\mathrm{IgG}$ are negative but there is a high index of suspicion (like extrahepatic features of autoimmunity/family history of autoimmunity or autoimmune diseases like vitiligo, thyroiditis) [1, B].

2.1.3.3. Frequency/degree of fibrosis may define chronicity (ACLF or ALF), but fibrosis may progress in a few weeks from F0 to F1-2 [2, C]. 
2.1.3.4. Corticosteroid therapy should be considered for a select group of patients presenting with ACLF-AIH [2, B].

2.1.4. Acute variceal bleed.

2.1.4.1. The frequency of acute variceal bleed (AVB) increases with severity of cirrhosis $[2, \mathrm{~B}\}$.

2.1.4.2. AVB in compensated cirrhosis (Child A) leads to the development of ACLF in less than $5 \%$ cases $[2, \mathrm{~B}]$.

2.1.4.3. Mortality in compensated cirrhosis increases with the development of ACLF in 90 day follow-up postvariceal bleed [2, B].

2.1.4.4. Incidence of post-EVL ulcers in ACLF is higher than that in cirrhosis $[1, \mathrm{C}]$.

2.1.4.5. Though infrequent, AVB can lead to ACLF in small proportion of Child A patients. Further studies are required in patients with Child $\mathrm{B}[2, \mathrm{C}]$.

2.1.5. Vascular liver diseases (PVT, HVOTO).

2.1.5.1. In patients with cirrhosis, development of acute occlusive portal vein thrombosis (PVT) may precipitate ACLF in a small sub-group $(2, \mathrm{C})$.

2.1.5.2. In patients with cirrhosis or Budd-Chiari syndrome, development of acute hepatic vein thrombosis (PVT) may precipitate $\operatorname{ACLF}(2, \mathrm{C})$.

2.1.5.3. Patients with Budd-Chiari syndrome (BCS) may infrequently present with or develop $\operatorname{ACLF}(2, \mathrm{C})$.

2.1.5.4. Evaluation of thrombophilic disorders in patients with PVT or BCS and ACLF should be similar to those without ACLF $(2, \mathrm{C})$.

2.1.5.5. There is no evidence currently to suggest that noncirrhotic portal fibrosis or EHPVO may present as $\operatorname{ACLF}(2, \mathrm{C})$.

2.1.5.6. No data are available about the natural history or outcome of patients with PVT or BCS presenting with ACLF and no recommendations can be made for management of patients with vascular liver diseases and $\operatorname{ACLF}(2, \mathrm{C})$.

\section{Defining the underlying chronic liver disease}

Two aspects were carefully analyzed, what constitutes chronic liver disease, cirrhosis alone or non-cirrhotic chronic liver diseases and the etiology of the chronic liver disease.

The degree of hepatic fibrosis and the functional hepatocellular mass remains heterogeneous in patients with the chronic hepatitis [53, 54]. Even in patients with stage IV fibrosis, critical mass varies according to the parenchymal reserves. Modified Laennec Scoring System divides stage IV further, according to the thickness of septa into three, ending up in six stages altogether $[55,56]$. Moreover, ACLF is not equivalent to the acute decompensation of cirrhosis, which has protean manifestations. Majority of the ACLF patients present with liver failure without any previous assessment of liver disease. It is not possible to distinguish accurately the natural history of patients with different degree of fibrosis presenting with ACLF at this point in time. The liver with any significant degree of fibrosis, with activated stellate cells, and infiltrated by the inflammatory cells, is expected to respond in a different way to the acute insult compared to the liver without inflammatory infiltrate [57].

NAFLD is the leading cause of donor rejection in liver transplantation [58]. Experience from liver transplantation centers shows that steatosis greater than $30 \%$ in the donor liver is associated with a higher risk of primary non-function and graft initial poor function as compared to grafts with no or less than $30 \%$ steatosis [59]. Patients with metabolic syndrome and fatty liver, diabetics, male patients of age greater than 45-50 years, and patients with obesity and dyslipidemia have increased risk of fibrosis [60]. While cirrhosis could be a late event, a large proportion of patients may have stage 2 or 3 fibrosis. Hence, NASH is indeed an important cause of chronic liver disease [61]. Furthermore, in the East, a large proportion of patients do have reactivation of chronic hepatitis B. In these patients, while liver failure and ACLF-like presentation do develop, cirrhosis is not necessarily present. The AARC data based on the liver biopsy studies corroborated the facts that a fair proportion of patients with ACLF do not have full-fledged cirrhosis, but still carry a poor prognosis, with mortality above $33 \%$ at 4 weeks. Based on the current data set, and published literature and the validity of the 2009 consensus on including the non-cirrhotic chronic liver disease were reaffirmed.

Accurate and reliable assessment of underlying CLD in the setting of ACLF is important for the subsequent management and need for liver transplant in these patients. Diagnosis of chronic liver disease in the setting of ACLF is made by history, physical examination, and previously available or recent laboratory, endoscopic or radiological investigations [62]. Ultrasound and CT abdomen may pick up CLD. However, to assess the degree of fibrosis in an un-shrunken liver would require other radiological modalities. The current non-invasive tests cannot clearly diagnose the presence of chronic liver disease in the presence of inflammation and liver failure. Hence, liver biopsy through the transjugular route or occasionally through laparoscopy remains an important tool to confirm the stage of fibrosis and presence of cirrhotic or non-cirrhotic liver disease.

A liver biopsy through the transjugular route may be of help when the presence of already underlying CLD and the cause of liver disease are not clear. The liver biopsy may highlight the etiology, stage of fibrosis, prognosis and outcome in patients with ACLF [63]. In addition, transjugular access directly into the hepatic vein allows the hepatic venous pressure gradient to be measured (HVPG). There is a risk of bleeding leading to hemobilia, hemoperitoneum, and hepatic hematoma in the setting of the deranged clotting profile [64]. The need of liver biopsy in ACLF should, therefore, be individualized. Standardization of liver biopsy 
assessment would help a uniform approach to the diagnosis and treatment of CLD and the acute insult.

There is a need to have reliable non-invasive tools to assess the severity of fibrosis in a previously undiagnosed CLD. Ultrasound and CT abdomen may pick up CLD. However, to assess the degree of fibrosis in an unshrunken liver would require other radiological modalities. Transient elastography is a good modality to detect hepatic fibrosis [65]. However, the liver tissue stiffness may also increase with hepatitis, steatosis, and inflammation present in the ACLF setting [66].

The second issue was about the etiology of chronic liver disease and cirrhosis in the Asian pacific region. The experts reviewed the data from the AARC database and the etiologic profile of cirrhosis in ACLF was found to be similar to etiology of cirrhosis in general in the respective countries [28, $67,68]$. With the rising incidence of obesity and NAFLD, a proportion of burnt-out NASH presenting as cryptogenic cirrhosis also increases [69-71]. Viral serology and nucleic acid testing are required to identify viral etiology. Specialized tests to diagnose metabolic and autoimmune diseases are needed as well. The presence of stigmata of liver disease on clinical examination, low platelets, evidence of synthetic dysfunction in previous reports, and altered AST/ALT ratio in previous reports should prompt the diagnosis of the presence of CLD [72, 73].

\section{Recommendations}

\subsection{Defining the underlying CLD:}

2.2.1. Cirrhotic and non-cirrhotic chronic liver diseases qualify as chronic liver diseases $(1, \mathrm{~A})$.

2.2.2. The common underlying chronic liver diseases include alcohol, hepatitis B, hepatitis C, NAFLDrelated chronic liver disease or cirrhosis of the liver $(1, \mathrm{~A})$.

2.2.3. Chronic hepatitis and/or significant fibrosis without cirrhosis should be taken as a chronic liver disease, if such a patient presents as $\operatorname{ACLF}(1, \mathrm{~B})$.

2.2.4. NAFLD-related chronic hepatic injury; NASH, if associated with significant fibrosis, should be taken as a chronic liver disease in $\operatorname{ACLF}(1, \mathrm{~B})$.

2.2.5. Patients with known previous decompensation with jaundice, HE, and ascites should be excluded $(1, \mathrm{C})$.

2.2.6. Diagnosis of chronic liver disease and cirrhosis in the setting of ACLF is made by history, physical examination, laboratory, endoscopic or radiological investigations $(1, \mathrm{~A})$.

2.2.7. A liver biopsy through the transjugular route may be helpful when the presence of underlying chronic liver disease and/or the cause of chronic liver disease and/or the acute insult is not clear $(1, \mathrm{~B})$.

\section{Impact of comorbidities and obesity}

Comorbidities also influence the outcome of ACLF as far as the disease and outcome are concerned. The presence of comorbidities like obesity, sarcopenia and other metabolic risk factors like diabetes mellitus, hypertension and dyslipidemia have a bearing on the outcome of patients with cirrhosis [74]. However, there is a sparse literature on the effect of obesity, sarcopenia and other metabolic risk factors on the severity and outcome of patients with acute-on-chronic liver failure (ACLF). In a recent analysis of the AARC database, the prevalence of metabolic risk factors and its impact on the severity and outcome were analyzed in patients with alcoholrelated ACLF as per the APASL definition [75]. In a recent report, of the 1028 patients from AARC database, $15 \%$ patients had history of overweight or obesity, $14 \%$ of T2DM, $7 \%$ of HT and $15 \%$ of dyslipidemia. Patients with metabolic traits compared with control group, had more severe disease; those with overweight or obesity had significantly higher MELD score and those with dyslipidemia had higher AARC score. None of the other metabolic risk factors either alone or in combination had any impact on the severity of ACLF. The presence of overweight or obesity was also significantly associated with increased day 30 mortality while none of the other metabolic risk factors, alone or in combination were associated with day 30 or 90 mortality [75]. In addition to above, alcohol intake and subsequent chronic liver disease with or without cirrhosis is another co-morbid condition.

\section{Recommendations}

\subsection{Impact of comorbidities and obesity.}

2.3.1. The presence of overweight or obesity and dyslipidemia increases the severity of liver disease in ACLF patients (1, B).

2.3.2. The presence of overweight or obesity increases the short-term (30 day) mortality in patients with $\operatorname{ACLF}(1, \mathrm{~B})$.

2.3.3. There is need to compare the development and natural history of ACLF in patients with NASH versus NASH cirrhosis $(2, \mathrm{C})$.

\section{Changing trends for the etiology of acute insult and chronic injury}

The epidemiology of acute insult has changed significantly in the past 5 years. In the recent AARC data, alcohol has now emerged as the most common etiology for acute insult (49\%) as well as for underlying chronic liver disease in contrast to previuos data of HBV predominance. DILI and autoimmune etiologies have shown increasing trend; however, HAV/HEV had decreasing trend. HBV infectioninduced ACLF as well as HAV/HEV-induced ACLF is now 
showing a decreasing trend over time, whereas alcohol and herbs, drugs and supplements (HDS)-induced ACLF show an increasing trend. The unknown causes for acute insult and chronic injury constitute only $5-15 \%$ cases of ACLF in the East in contrast to the West, where these are seen in about $40 \%$ of ACLF patients.

\section{Recommendations}

\subsection{Changing trends for the etiology of acute insult} and chronic injury.

2.4.1. Alcohol is now the commonest etiology for acute hepatic insult as well as for the underlying chronic liver disease in the Asian continent [2, B].

2.4.2. DILI and autoimmune etiologies have shown increasing trend $[2, \mathrm{~B}]$.

2.4.3. HBV infection-reactivation of hepatitis B-induced ACLF as well as acute $\mathrm{HAV} / \mathrm{HEV}$-induced ACLF shows a decreasing trend over time in certain regions, whereas alcohol and herbs, drugs and supplements (HDS)-induced ACLF show an increasing trend $(1, \mathrm{~A})$.

2.4.4. The unknown causes for acute insult and chronic injury constitute only $5-15 \%$ cases of ACLF in the East in contrast to the West, where these are seen in about $40 \%$ of ACLF patients $(1, \mathrm{~A})$.

\section{ACLF is distinct from acute decompensation (AD): differentiating $A D$ and $A C L F$}

The two disease entities look similar and are often misunderstood. The experts reviewed the literature and presented their data. The data from the AARC database were presented. The discussion revolved around the following main issues:

Acute decompensation occurs in a cirrhotic with or without prior decompensation and is often associated with a precipitant [6]. The presentation of $\mathrm{AD}$ is either hepatic (jaundice, ascites, HE) or extrahepatic (variceal bleed, acute kidney injury or sepsis), and time period is up to 3 months [11]. The level of jaundice could be well below $5 \mathrm{mg} / \mathrm{dl}$, below the cutoff generally taken for liver failure. The precipitant for AD can be hepatic (48\%) or non-hepatic (46\%). Ascites/HE/AVB may precede jaundice. There could be several combinations in the acute decompensation; such as jaundice with or without ascites, HE alone or with ascites with or without jaundice, HE variceal bleed alone or with ascites, sepsis with jaundice or alone, etc. Each of these entities is in themselves, a well-defined complication, and has been extensively studied in patients with cirrhosis. Moreover, $\mathrm{AD}$ can be the index event or it could be a repeat event in patients with prior decompensation. Hence, there are multitudes of combinations possible in a patient presenting with
$\mathrm{AD}$. After due deliberations, it was unanimously felt that $\mathrm{AD}$ should be considered as a recordable time point, an unfavorable event, in the natural history of cirrhosis rather than a syndrome by itself. The precise type of acute presentation of the patient should be recorded and the patient should be accordingly monitored and managed.

The overall mortality in patients with $\mathrm{AD}$ at 1 and 3 months was $23 \%$ and $29 \%$, respectively, much lower than when patients develop ACLF. The probability of reversal, progression to end-stage liver disease and need for a liver transplantation would depend on the presentation of the $\mathrm{AD}$ such as variceal bleed or ascites. Role of bridging therapy and emerging therapies in $\mathrm{AD}$ is largely unknown.

Acute-on-chronic liver failure (ACLF) is a syndrome of hepatic decompensation (jaundice, coagulopathy, ascites and/or HE), where the insult is only hepatic and leads to liver failure in a period of 4 weeks [2]. Jaundice and coagulopathy precede development of ascites. Non-hepatic organ failure, i.e., AKI, sepsis, AVB develops after the ACLF syndrome or less commonly, with the onset, depending on the severity of liver failure. The presentation is index, occurring in a patient of chronic liver disease with or without underlying cirrhosis of the liver. The hepatic reserve may show recovery leading to complete reversal of the syndrome as well as regression of fibrosis and portal hypertension. The long-term survival, i.e., after 24 months of index presentation with ACLF is better than the AD cohort [HR: 1.94 (1.17-2.21), $p<0.01$ ] [76]. The progression of disease and onset of multi-organ failure are faster in the non-salvageable cohort with a high 3-month mortality of 54\% [77].

Development of ascites represents a state of acute portal hypertension in ACLF patients. This rapid rise in portal pressure is a result of severe hepatic inflammation and ongoing liver failure. Highly activated stellate cell population, cytokine storm and ongoing hepatic parenchymal necrosis perpetuate the portal hypertension syndrome. The use of non-selective beta-blockers has been shown to be effective in reducing the mortality and risk of variceal bleed in ACLF patients.

ACLF is a unique disease entity and is distinct from acute decompensation by considering only those patients who have one type of AD and in a specified time frame of 28 days; this includes patients who develop after a hepatic insult, jaundice and coagulopathy followed by development of acute portal hypertension in the form of ascites and or HE (Table 3 ).

\section{Recommendations}

\subsection{ACLF is distinct from acute decompensation (AD): differentiating AD and ACLF.}




\subsubsection{Natural history and outcome of ACLF.}

2.5.1.1. The main etiologies for ACLF are alcohol-related injury, viral hepatitis, drug-induced liver injury, and autoimmune liver disease. In the Asian Pacific region, in only about $5-10 \%$ of the cases, the acute insult is unidentifiable [1, A].

2.5.1.2. Age and the presence of cirrhosis are independent risk factors for mortality in ACLF $(1, B)$.

2.5.1.3. Portal hypertension with an HVPG greater than $18 \mathrm{mmHg}$ and/or variceal bleeding, presence of complications including ascites, SBP and encephalopathy are independent predictors for mortality (2, B).

2.5.1.4. Starting NSBBs is safe in ACLF and its use is associated with improved short-term survival $(2, \mathrm{~B})$.

2.5.1.5. Appropriate management has key impact on the outcomes of ACLF, early (within 2 weeks) antiHBV treatment for HBV-ACLF, corticosteroid therapy for alcoholic ACLF and AIH-ACLF are worthwhile options $(1, \mathrm{~B})$.

\subsubsection{Natural history and outcome of acute decompen-} sation.

2.5.2.1. Acute decompensation (AD) is currently defined as acute occurrence of decompensating events (ascites, HE, jaundice, variceal bleed or bacterial infection) in a patient with CLD $(1, \mathrm{~B})$.

2.5.2.2.Patients with $\mathrm{AD}$ who have or progress to develop extrahepatic organ failure have high short-term mortality $(1, \mathrm{~A})$.

2.5.2.3.Early evaluation of potential predictors and precipitating agents can help in managing these patients $(1, \mathrm{~B})$.

\subsubsection{Acute decompensation-differentiating from ACLF for clarity in definition.}

2.5.3.1. AD develops in a patient with chronic liver disease/ cirrhosis, with or without prior decompensation, and is often associated with an identifiable precipitant and develops in less than 3 months [2, A].

2.5.3.2. Any decompensation preceding jaundice strongly favors $\mathrm{AD}[1, \mathrm{~B}]$.

2.5.3.3. Absence of repeated episodes of decompensation differentiates ACLF as a unique syndrome [2, A]. This has implication on the management decisions and prognostication, including reversibility of the syndrome.

2.5.3.4. Long-term survival, reversal and/or recovery of hepatic reserve has been documented with ACLF $[2, \mathrm{~A}]$.

2.5.4.5. The differentiating features between different presentations of $\mathrm{AD}$ and the ACLF need to be studied carefully by expanding the AARC database $[3, \mathrm{C}]$.

\section{Role of Liver histology in ACLF}

Since the previous consensus statement, new data and insights into the liver histopathology have become available. The main questions that were addressed in the current consensus meeting were: (1) Is liver biopsy feasible and safe in ACLF, (2) Can liver biopsy help to differentiate ACLF from ALF and chronic liver disease, (3) Are there any histological predictors of outcome in ACLF, such as need for liver transplantation or mortality and (4) Are there any differences in regenerative response in sequential biopsies of survivors and non-survivors?

Percutaneous liver biopsy is generally not feasible in patients with ACLF due to coagulopathy and ascites. Transjugular liver biopsy (TJLB), on the other hand, is considered relatively safe and can help assess stage of fibrosis and severity of hepatic injury. For example, severity of alcoholic hepatitis in alcoholic liver disease-related ACLF could be assessed only by liver biopsy [63]. It can provide clues to the underlying acute insult as in Wilson's disease, malignancy, autoimmune hepatitis, DILI and NASH [78, 79].

Table 3 Differentiating ACLF from acute decompensation

\begin{tabular}{lll}
\hline Parameter(s) & Acute-on-chronic liver failure (ACLF) & Acute decompensation (AD) \\
\hline Presentation & Hepatic insult & Hepatic or non-hepatic \\
& Index & Can be index or subsequent \\
Identifiable precipitant & In up-to 95\% cases & In to 70\% cases \\
Time from insult to presentation & Within 4 weeks 12 weeks \\
Underlying cirrhosis & May or may not be present & Always present \\
Prior decompensation & No & With or without Prior Decompensation \\
Mortality at 1 and 3 months & $33-51 \%$ & $23-29 \%$ \\
Reversal or recovery & In half of cases & Uncommon \\
\hline
\end{tabular}


Mini-laparoscopic liver biopsy is another alternative for getting liver biopsy in patients of advanced cirrhosis with acceptable bleeding risk. More data needed on this modality and comparison with TJLB are lacking at present. However it can be considered in areas with poor access to TJLB and biopsy is definitely needed for the decision- making [80].

Differentiating ALF and chronic hepatitis with flare is based on findings of fibrous bands (spurs and bridges) and ductular proliferation. Features of cholestasis and bile duct proliferation are more common in patients with acute injury (classical features of acute hepatitis along with cellular and ductular cholestasis are indicative of acute injury). Differentiation between cirrhosis with acute deterioration and compensated cirrhosis is based on the presence of necrosis and features of acute hepatitis in the former group of patients [63]. It was proposed that the diagnostic stains for fibrosis and necrosis be mentioned. It was also proposed that connective tissue stains (especially Shikata's orcein) should be done in all such cases for differentiating necrosis from fibrosis.

Liver histopathology could be very useful in prognosticating the outcome in a patient with ACLF $[63,81]$. The extent of necrosis, liver damage and fibrosis is helpful. The presence of ductular bilirubinostasis on liver biopsy defined as the presence of bile plugs in dilated ductules at the interface between the portal tract and parenchyma predicted a poor outcome and a high potential for development of infections in ACLF. While ballooning was helpful, suggestive of regenerating potential, the presence of eosinophilic degeneration of hepatocytes was not a favorable feature. Standardization of liver biopsy assessment is essential for a uniform approach to the diagnosis and treatment of CLD and acute insult.

Liver regeneration is considered to play an important role in ACLF as prognosis can be improved if the critical threshold of functional liver cell mass is regained. Decompensated cirrhosis is considered irreversible owing to loss of regeneration potential. Liver histology can provide morphological evidence supporting these concepts and for assessing regenerative potential and prognosis [82]. In this report, immuno-histochemical study of two levels of regenerative responses in liver failure revealed that proliferating hepatocytes were significantly more in ALF in comparison to $\operatorname{ACLF}(p<0.001)$ and CLD $(p<0.001)$.

There is significant relationship between HSCs and presence of HPCs, indicating a possible dynamic role of HSCs in liver regeneration and pathobiology of ACLF [83]. Liver biopsy is an important mode of understanding and validating the results of clinical trials exploring various therapeutic options for, e.g., mobilization of bone marrow-derived stem cells with granulocyte colony-stimulating factor (GCSF) [84].

\section{Recommendations}

\subsection{Role of liver histology in ACLF.}

2.6.1. A liver biopsy through the transjugular route is helpful to diagnose/confirm the cause of acute injury $[1, \mathrm{~B}]$.

2.6.2. Liver biopsy is helpful in patients where the presence and stage of underlying chronic liver disease and/or the cause of chronic liver disease are not clear (2, A). Biopsy can help identify unsuspected/ multiple etiologies, i.e., primary and concomitant etiologies.

2.6.3. The need of liver biopsy in ACLF should be individualized, especially in alcoholic hepatitis, severe autoimmune hepatitis, and flare of Wilson disease $(2, \mathrm{~A})$.

2.6.4. Liver biopsy indicates the stage of fibrosis and is helpful in the prognosis and outcome in patients with ACLF (B 1). It can help in distinguishing ACLF from decompensated cirrhosis [1, B].

2.6.5. Certain histologic parameters are predictors of prognosis of ACLF, like ductular bilirubinostasis, eosinophilic degeneration, and parenchymal extinction $(1, \mathrm{~B})$.

2.6.6. It can help in distinguishing ACLF from decompensated cirrhosis [1, B].

2.6.7. Standardization of liver biopsy assessment is essential for a uniform approach to the diagnosis and treatment for CLD and acute insult $(2, \mathrm{C})$.

2.6.8. Noninvasive tools to measure liver stiffness and biomarkers may help in identifying patients with advanced fibrosis. Studies are needed to validate the performance of these tests in the setting of $\operatorname{ACLF}(2, \mathrm{C})$.

\section{Defining the liver failure in ACLF}

Acute liver failure is generally defined as development of HE within 4 weeks of onset of jaundice [1,2]. Since the basic premise in ACLF is to identify patients with chronic liver disease or cirrhosis presenting as acute liver failure, the time frame for liver failure was kept as 4 weeks [5].

The clinical presentations in ACLF is varied and depends upon the severity of acute insult and degree of underlying chronic liver disease. In the published reports, patients included as having ACLF had severe jaundice associated with organ failure manifested as either HE or hepatorenal syndrome (HRS) [1, 2].

Defining the liver failure in ACLF, therefore, required a detailed consideration of all the existing liver failure scores and the criteria defining liver failure in the organ failure scores such as SOFA and APACHE II. The two main variables are bilirubin and coagulopathy. 
Serum bilirubin analysis of the AARC data revealed that patients with a bilirubin between 5 and $10 \mathrm{mg} / \mathrm{dl}$ also had substantial mortality ranging around $38 \%$. The data for patients below this level were, however, not collected as per the initial definition, but is likely to yield mortality rates much below $33 \%$. On the other hand, in the CANONIC study, the level of bilirubin for hepatic failure was taken as $12 \mathrm{mg} / \mathrm{dl}$ so as to determine $15 \%$ mortality at 28 days. If these criteria were applied to the ACLF patients in the Asian region, a much higher mortality was observed in this cohort. After detailed discussion, the original value of $>5 \mathrm{mg} / \mathrm{dl}$ was accepted as the cutoff for bilirubin for defining liver failure $[1,2]$. This was reiterated to be inclusive of less severe group of patients and enabling a complete spectrum of patients, including those who have a potential for recovery [2].

Coagulopathy the presence and degree of coagulopathy as a marker for liver failure were re-evaluated. Coagulopathy is an important hallmark of severe hepatic dysfunction [59, 60]. Patients with ACLF have complex hemostatic defects leading to a delicate, unstable balance between bleeding and thrombosis [85].

Development of clinical ascites and/or encephalopathy has been conventionally taken as evidence of hepatic failure $[1,27]$. Ascites and HE were not seen in all the patients and, therefore, presence of either of them was accepted for the definition of ACLF. In the AARC data, ascites was present in $91 \%$ and $\mathrm{HE}$ in about $45 \%$ of the patients at presentation.

The data were further analyzed to see if a shorter interval of 2 weeks instead of 4 weeks is a better cutoff for predicting mortality in patients with underlying cirrhosis who developed jaundice followed by ascites. The analysis of the AARC data showed that in patients who developed ascites within 2 weeks against those after 2 weeks of onset of jaundice, though had a slightly more severe course, the differences in mortality were not significant.

Grade of liver failure for ACLF like in many conditions in medicine, such as the NYHA classification for heart failure [86] severity of a disease or variable can be defined to predict the outcome of the disease. Using the four variables, bilirubin, INR, ascites and HE, a simple scoring system may be helpful for making treatment strategies.

The basic premise for defining a syndrome is to identify a group of patients, who have a distinct presentation, course and outcome. A prospective study using AARC database with 1402 patients from several centers across Asia included a large derivation cohort of 480 patients to develop a dynamic prognostic model, which was validated in subsequently enrolled 922 patients to predict outcomes including mortality [3]. The results bring forth a simple 'liver failure grading system' for patients with chronic liver disease based on variables, namely serum bilirubin, INR, grade of HE, serum lactate and serum creatinine [43, 87-89]. Serum lactate levels are elevated in relation to degree of hepatocellular injury, inflammation and degree of tissue perfusion.

The analysis resulted in a simple "liver failure grading system' based on 5 variables; namely, serum bilirubin, INR, serum lactate, serum creatinine and grade of HE. There is no score dedicated to liver failure in cirrhotic patients, commonly recognized as a distinct entity, ACLF. The grading system, i.e., Grade I for a score of 5-7, Grade II for 8-10 and Grade III for 11-15 with 28-day mortality of 12.7, 44.5 and $85.9 \%$, respectively, was developed. The grades of liver failure showed a potentially recoverable group (Gr. I), a group that needs special monitoring (Gr. II) and a group that demands immediate interventions for improved outcome (Gr. III). The AARC model was found to be better than existing models for ACLF with an excellent predictability, i.e., in AUROC of 0.80 (derivation cohort) and 0.78 (validation cohort). It is even more robust than recently reported models [3] where the AUROC is below 0.80. The AARC-ACLF score is dynamic in nature. It could predict day 7 (score of 9 or below) and day 28 survival at presentation (score of 9 or below). For a baseline score of $\geq 10$, with each one unit increase, the day 7 mortality increased sharply compared to the patients who presented with a score of $<10$ (20\% vs. $4 \%)$. The score also predicted well the day 28 and day 90 survival. Thus, the AARC score provides a physician a window to decide early and explore definitive therapies including liver transplantation. Shift from grade I to III liver failure at day 4 and day 7 increases the mortality significantly. At the same time, the persistence of grade I or II liver failure till day 7 predicted improved survival, while persistence in grade III failure was uniformly severe and warranted early consideration for transplant [3].

\section{Recommendations}

\subsection{Defining the liver failure in ACLF:}

3.1. Jaundice (serum bilirubin $>5 \mathrm{mg} / \mathrm{dL}$ [ $>85 \mathrm{lmol} / \mathrm{L}]$ ) and coagulopathy (INR $>1.5$ or prothrombin activity $<40 \%)$ are mandatory parameters to assess liver failure $(1, \mathrm{~A})$.

3.2. Ascites and/or encephalopathy as determined by physical examination also reliably reflect significant hepatic functional impairment $(1, \mathrm{~A})$.

3.3. Liver failure score (AARC score) which includes total bilirubin, INR, grade of HE, plasma lactate and serum creatinine reliably predicts the disease severity and outcome $(1, \mathrm{~A})$.

3.4. Grading of liver failure as per AARC score I (5-7), II (8-10), III (11-15) effectively prognosticates and guides the therapy $[1, \mathrm{~B}]$.

3.5. The assessment of coagulation system by global coagulation methods (viscoelastic technique/thrombin genera- 
tion test) may be considered as a useful tool for assessing coagulation anomalies in ACLF patients $(2, \mathrm{~B})$.

\section{Sepsis in ACLF}

Sepsis is a syndrome of systemic inflammatory response of the host to an identifiable infection. The systemic inflammatory response syndrome (SIRS) is defined by the presence of at least two of the following criteria: (1) altered temperature, (2) elevated respiratory rate or hyperventilation, (3) tachycardia, and (4) altered white blood cell count (high, low, or immature forms) [67]. Sepsis is the most common cause of mortality in most intensive care units (ICUs) [90-92].

Due to the hyperdynamic circulation and complications of portal hypertension, the currently accepted clinical definition of SIRS and sepsis may not be entirely applicable to patients with cirrhosis or ACLF. Hence, a high index of suspicion is required for making a clinical diagnosis of sepsis in these patients. Liver failure initiates and predicts the development of SIRS. New onset SIRS in the first week is an important determinant of early sepsis, organ failure, and survival (Fig. 3). Prompt interventions in this 'golden window' before development of sepsis may improve the outcome of ACLF [78].

Consideration of sepsis as an acute insult in the absence of liver failure is confusing and with limited scientific basis. Sepsis is a consequence rather than the cause for development of ACLF. The APASL definition does not include sepsis as a primary cause for liver failure, but in the Western definition, sepsis is considered as the most common precipitant. The inclusion of sepsis in the definition is likely to be associated with concomitant multi-organ involvement, poor prognosis and would be unlikely to provide a targeted therapy or a definitive therapy such as liver transplant.

SIRS is the inflammatory response to the damaged organ in the host. It could be a result of sterile inflammation or an occult infection [93-95]. In fact, in $60 \%$ of patients fulfilling the SIRS criteria, infection could not be detected [78]. This highlights the limitations of the current techniques available to detect infections or may be because of use of prophylactic antibiotics the detection of organisms becomes difficult. Prevention of development of SIRS or its progression from SIRS to sepsis by immune modulation in the 'Golden Window' period could decrease the incidence of organ failure and improve survival [78]. 'Golden window' is a short period of about 1 week before the onset of sepsis and development of extrahepatic organ failure in a patient with ACLF. Therapeutic interventions during this period are likely to prevent organ failure and provide a potential opportunity for ameliorating or reversing the hepatic injury and failure [78].

The current paradigm regarding the host immune response to sepsis is debated and is a matter of great interest in clinical trials as well as basic science. Two theories have been proposed to describe the host response to sepsis. According to the most accepted theory, both proinflammatory and anti-inflammatory responses occur early and simultaneously in sepsis although the net initial effect of these competing processes is typically manifested by an early, dominant, hyperinflammatory phase characterized by shock, fever and hypermetabolism [96, 97]. Subsequently, this initial hyperinflammatory phase evolves over several days into a more protracted immunosuppressive phase [98]. The robustness of the hyperinflammatory phase depends on numerous factors, including pre-existing co-morbidities, nutritional status, microorganism load and virulence factors [99, 100].

According to the second theory, there is rapid and sustained upregulation of genes that regulate the innate immune response and the simultaneous downregulation of genes that regulate the adaptive immune response. There is protracted, unabated inflammation driven by the innate immune system with resultant organ dysfunction and failure [101].

Whether sepsis is the cause or a result of liver failure was debated at length. The fact that patients who presented with no SIRS or subsequently developed SIRS or sepsis over a period of 1-2 weeks indicates that infection and sepsis develop after liver failure and unabated inflammation leads to immune paresis and provides an opportunity for infections and sepsis to occur. Non-hepatic infections are also common in patients with ACLF [101, 102]. Neutrophil dysfunction and immune paralysis due to reduced HLA-DR expression have been shown to rapidly develop in ACLF patients [102-104]. The frequency of intrahepatic myeloid and plasmacytoid dendritic cells is reduced with increased interferon gamma producing CD8 $\mathrm{T}$ cells in patients with

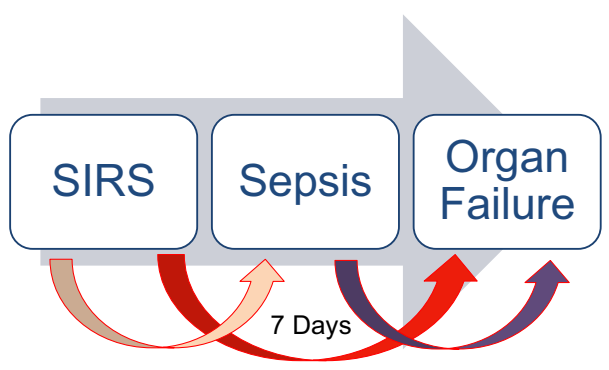

Fig. 3 Golden window in ACLF. ACLF is the state of acute inflammatory response with cytokine burst. The SIRS is a response to this inflammation and subsequent resolution of inflammation and recovery or persistence of inflammation (leading to Compensatory Antiinflammatory Inflammatory Response Syndrome-CARS) and sepsis. Patients of ACLF in a period of 7 days develop SIRS (which can be infective or sterile) but both the things lead to complications and sepsis develops subsequently. This time period is the therapeutic Golden Window. SIRS needs consideration for organ support, antibiotics for occult sepsis and prioritization for definitive therapy, i.e., liver transplant 
ACLF. Decreased frequency of DCs and high IFN- $\gamma$ levels correlate with poor patient survival [104].

Bacterial infection is present in about $1 / 3$ rd of ALCF patients at presentation to a tertiary referral hospital, and this further increases by first week [78]. The AARC data showed that the patients presenting with sepsis, at baseline or who developed new sepsis at day 4, have high mortality. Bacterial infection (BI) predicts development of organ failure in ACLF. Organ dysfunction and organ failure are significantly higher in infected cohort with high short-term mortality. The acute phase proteins, such as $\mathrm{C}$-reactive protein and procalcitonin, were proven to be reliable biomarkers for bacterial infection. The most frequent infections are SBP, pneumonia, UTI, and bacteremia. Second infection (2nd hit) is associated with poor outcome in patients with ACLF. Hospitalized patients with ACLF should be monitored closely for the presence of infections to enable early diagnosis and treatment. Routine examination of blood and body fluids is recommended. Patients who respond to treatment for bacterial infection have significantly reduced mortality. Patients who respond to treatment for bacterial infection have significantly reduced mortality. As soon as bacterial infection is diagnosed or suspected, broad spectrum antimicrobial agents or combined use of antibiotics are preferred, thereafter the therapy is adjusted according to the results of the sensitivity test. Empirical antibiotic therapy should be based on environment, local resistance profiles, severity and type of infection. To optimize the empirical antibiotic treatment, it is quite important to distinguish among community acquired, health care associated and nosocomial infections.

Invasive fungal infection is not uncommon in ACLF patients. However, data are minimal. The diagnosis of invasive fungal infection can be proven, probable or possible, depends on mycological evidence, and clinical evidence. Invasive pulmonary aspergillosis (IPA) is increasingly recognized as a cause of morbidity and mortality in patients with ACLF. Prophylaxis of invasive fungal infection may be applicable with echinocandins in selected patients. Prophylaxis with fluconazole followed by echinocandins needs to be evaluated in ACLF patients. Predictors of poor progression (risk factors) include diabetes, AKI, ICU admission, and admission for bacterial infection, prolonged antibiotic therapy ( $>5$ days from admission), prior $\mathrm{h} / \mathrm{o}$ hospitalization. Biomarkers such as galactomanan or B-D Glucan can be used for supporting diagnosis if there is invasive fungal infection in ACLF. Administration of albumin is recommended in patients with SBP for preventing from type-1 HRS and reducing mortality. The role of albumin in preventing or treating other infections in ALCF is not clear.

\section{Recommendations}

\subsection{Sepsis in ACLF.}

4.1. There is a central role of inflammation and dysbalance of innate and adaptive immune responses in ACLF patient $(1, \mathrm{~B})$.

4.2. It is difficult to differentiate SIRS from early sepsis in cirrhosis $(2, \mathrm{~A})$.

4.3. Bacterial infection is present in about $1 / 3$ rd of ALCF patients at presentation to a referral hospital, and this may increase in the first week [2, B].

4.4 Patients who do not have sepsis have low 28-day mortality [B2]. Patients with accompanying sepsis at baseline or who develop sepsis at day 4 have high mortality $[2, \mathrm{~B}]$.

4.5 Organ dysfunction and organ failure are significantly higher in infected cohort and this is attended with high short-term mortality [2, B].

4.6 Bacterial infection (BI) is an important factor to predict development of organ failure in ACLF. The most frequent infections are SBP, pneumonia, UTI, and Bacteremia $(1, \mathrm{~A})$.

4.7 Second infection (2nd hit) is associated with poor outcome in patients with ACLF $[1, \mathrm{~B}]$.

4.8 Hospitalized patients with ACLF should be monitored closely for the presence of infections to enable early diagnosis and treatment. Routine examination of blood and body fluids is recommended $(1, \mathrm{~A})$.

4.9 The acute phase proteins, such as C-reactive protein and procalcitonin, have been proven to be reliable biomarkers associated with infection and are recommended for screening for the presence of bacterial infections (1, B).

4.10 As soon as bacterial infection is suspected or diagnosed, broad spectrum antimicrobial agents alone or in combination should be started and thereafter, the therapy should be adjusted according to the results of the antibiotic sensitivity test results $(1, \mathrm{~A})$.

4.11. Empirical antibiotic therapy should be based on environment, local bacterial resistance profiles, severity and type of infection. To optimize the empirical antibiotic treatment, it is quite important to distinguish among community acquired, health care associated and nosocomial infections (2, A).

4.12. Invasive fungal infection is not uncommon in ACLF patients. These can be proven, probable or possible, depending on mycological and clinical evidences (2, B). Biomarkers such as galactomanan or B-D glucan can be used for supporting the diagnosis (1, B).

4.13. Invasive pulmonary aspergillosis (IPA) is increasingly recognized as a cause of morbidity and mortality in patients with ACLF. Voriconazole plasma concentration monitoring may ensure the safety and efficacy of ACLF patients with Invasive aspergillosis infection (2, C). 
4.14. Prophylaxis of invasive fungal infection can be done using echinocandins in selected patients (B2). Prophylaxis with fluconazole followed by echinocandins needs to be evaluated in ACLF patients $[1, \mathrm{C}]$.

4.15. Predictors of poor progression (risk factors) of fungal infections in ACLF are the presence of diabetes, AKI, ICU admission, and admission with bacterial infection, prolonged antibiotic ( $>5$ days pre-admission) and prior hospitalization (2, B).

4.16. The value of qSOFA and Sepsis- 3 criteria is unclear to assess severity of infection in patients with ACLF.

4.17. Administration of albumin is recommended in patients with SBP to prevent development of type-1 HRS and reduce mortality (A2). The role of albumin in preventing or treating other infections in ALCF is not clear [2, B].

4.18. Patients who respond to treatment for bacterial infection have significantly reduced mortality $[2, \mathrm{~B}]$.

\section{Organ dysfunction and organ failure in ACLF}

The concept to differentiate organ dysfunction from organ failure is useful in assessing the degree of organ damage and predicting the probability of disease progression or regression; prioritizing the patient for liver transplantation and the likelihood of futility of care or high probability of death.

The data from AARC database were carefully analyzed with respect to defining organ dysfunction and organ failure for each of the extrahepatic organs.

\section{Renal failure}

Renal failure in patients of ACLF is considered to be a complex and challenging condition that is associated with an ominous prognosis. Further, kidneys are one of the most frequent extrahepatic organs involved in patients with ACLF. The EASL-CLIF consortium has defined ACLF in which kidney dysfunction is used as a defining condition [11]. Hence, renal dysfunction or failure is universally present in patients with ACLF with or without liver failure, according to the definition by the EASL-CLIF consortium. On the contrary; the APASL definition of ACLF does not incorporate kidney dysfunction in its definition $[1,2]$. Studies based on APASL criteria have reported renal dysfunction in $22.8-34 \%$ of patients with ACLF and as high as $51 \%$ using the more sensitive AKIN criteria [103]. This highlights the fact that a significant number of patients of ACLF based on APASL criteria who do not have renal dysfunction (using even the most sensitive criteria to detect renal involvement) would definitely be missed if renal dysfunction was considered in the definition as per CLIF.
In patients with decompensated cirrhosis, the main abnormality causing renal dysfunction is systemic and splanchnic vasodilatation secondary to portal (or sinusoidal) hypertension which leads to decreased effective arterial blood volume and activation of neurohormonal systems, the rennin-angiotensin aldosterone (RAAS), the sympathetic nervous system and non-osmotic release of antidiuretic hormone, resulting in sodium and water retention [104-106]. The systemic hemodynamic alterations in ACLF are similar to those in patients with decompensated cirrhotics [107], but the pathogenesis of renal dysfunction in ACLF is quite different; a major role is played by SIRS and subsequent sepsis in patients of ACLF but not in those with decompensated cirrhosis [108]. A higher prevalence of structural AKI has been reported for patients with ACLF. In a large cohort study from the AARC database, the concept of PIRO (defined as predisposition, infection/inflammation, response, organ failure) was used for creating a predictive model for understanding the pathophysiology of kidney dysfunction in patients with ACLF [109]. Together, the components of PIRO reflect the role of key factors, which result in development, and progression of AKI in patients with ACLF. The PIRO, which is a composite score, derived from the AARC database accurately predicted in patients with ACLF, highlighting the prognostic significance of kidney dysfunction in these patients. The PIRO score can predict the development of AKI within first 15 days of diagnosis of ACLF and may help in stratification for additional therapeutic interventions [109].

Serum creatinine is used for defining and staging AKI in patients with cirrhosis. However, serum creatinine is influenced by various factors, which affect its production and excretion, as well as those that impact its measurement and, therefore, is unreliable in patients with severe liver dysfunction including ACLF. The AKI criteria seem more relevant as they rely on a dynamic change rather than a static measurement of serum creatinine. A lower value of serum creatinine is more relevant in patients with ACLF. Serum creatinine above $0.7 \mathrm{mg} / \mathrm{dl}$ (as derived from the AARC score) has a sensitivity of $78 \%$ and specificity of $36 \%$ for prediction of 30-day mortality in patients with ACLF. For the diagnosis of kidney failure, the conventional cutoff of $1.5 \mathrm{mg} / \mathrm{dl}$ even though had a low sensitivity of $48 \%$ but had a specificity of $99.8 \%$ for 30 -day mortality. Kidney failure (serum creatinine $\geq 1.5 \mathrm{mg} / \mathrm{dl}$ ) was seen in $22 \%$ of ACLF patients at baseline and developed in another $30 \%$ within a month. The majority of patients of ACLF developed new episodes of AKI in the first 2 weeks (11\%). Further, the KDIGO definition for AKI (incorporating the urine output criteria) has been shown to be more accurate in determining AKI course in critically ill cirrhotics; however, this needs to be evaluated for patients with ACLF [110].

Apart from the severity, the course of AKI was seen to be an important predictor of clinical outcomes. Almost $80 \%$ 
of the patients, who did not present with AKI at baseline or developed within the first 7 days, survived 1 month. However, in patients who presented with AKI, resolution of AKI was associated with a better survival. Progression of AKI was associated with highest mortality of $75 \%$. Development of new AKI as well as non-resolution or persistence of AKI was associated with almost $50 \%$ mortality at 1 month in patients with ACLF [109].

Kidney injury biomarkers have been studied in patients with decompensated cirrhosis; however, their utility in diagnosing, staging and understanding the spectrum of AKI including the response to therapies has not been carefully evaluated in patients with ACLF. There is a potential to look at the role of biomarkers of tubular damage, namely $N$-GAL, Kim-1, IL-18 and L-FABP to differentiate functional AKI or HRS from structural AKI, i.e., ATN patients with ACLF [111] as ATN or structural kidney damage may necessitate the need of simultaneous liver-kidney transplant as against liver transplant alone for HRS [112].

Treatment for AKI in ACLF depends on the etiology, severity, complications, and the presence of other organ failure and/or hemodynamic status. Targeting the components of PIRO, i.e., combating systemic inflammation with antiinflammatory strategies (for instance intravenous albumin, $\mathrm{N}$-Acetylcysteine), bilirubin reduction, avoidance of nephrotoxic drugs, aggressive management of circulatory failure and maintaining a high mean arterial pressure may prevent AKI development and progression in patients with ACLF. Albumin intravenous administration has multiple benefits volume correction in addition to its anti-inflammatory and immunomodulatory properties.

Data on use of vasoconstrictors for AKI in ACLF are limited. Terlipressin given as an infusion is superior to noradrenaline in the management of HRS-AKI in patients with ACLF, but needs extra precaution and close monitoring for terlipressin-related adverse effects [111]. The response to vasoconstrictor drug is multifactorial and depends on the severity of AKI, MELD score and AARC grade [113, 114]. Response to vasoconstrictors in AKI in ACLF patients is partial and seen in only in about a third of patients. There is, therefore, quite often a need for renal replacement therapy (RRT). The indications for RRT include severe volume overload, hyperkalemia, hyponatremia and worsening metabolic acidosis not responding to conservative management [115]. Unlike other indications, the threshold for initiating RRT should be relatively low, i.e., when AKI occurs as part of multi-organ failure or in non-oliguric patients, if the daily fluid balance cannot be maintained, Continuous Renal Replacement Therapy [CRRT] should be considered [116]. The mode of dialysis, i.e., hemodialysis (HD), Slow Low Efficient Dialysis (SLED) or CRRT should be chosen based on the hemodynamic status of the patient and the expertise and availability of the equipment $[115,116]$.

\section{Recommendations}

\subsection{Renal failure in ACLF.}

\subsubsection{Defining AKI in ACLF.}

5.1.1.1. AKI criteria (defined as an absolute increase in serum creatinine of $0.3 \mathrm{mg} / \mathrm{dl}$ within $48 \mathrm{~h}$ or by percentage increase in serum creatinine of more than $50 \%$ from baseline, which is known, or presumed, to have occurred within the previous 7 days) should be used for the diagnosis of AKI in patients with ACLF $[1, \mathrm{~A}]$.

5.1.1.2. AKIN criteria should be used for the diagnosis and prognostication of AKI in ACLF patients $(1, \mathrm{~B})$.

5.1.1.3. Urine output should be considered to assess the stage and course of AKI in patients with ACLF admitted to the ICU $[2, \mathrm{C}]$.

5.1.1.4. In the absence of baseline serum creatinine, AKI should be diagnosed based on the cutoff value of serum creatinine. A cutoff value of $1.1 \mathrm{mg} / \mathrm{dl}$ is a reliable marker of significant renal dysfunction and $1.5 \mathrm{mg} / \mathrm{dl}$ of kidney failure in patients with ACLF $[1, \mathrm{~B}]$.

5.1.2. Course of renal failure in ACLF.

5.1.2.1. AKI is more common and rapidly progressive in patients with ACLF as compared to decompensated cirrhosis and is associated with significantly worse outcome (1, B).

5.1.2.2. Patients with ACLF and AKI have high 30-day mortality. The course of AKI is an important determinant of clinical outcomes $[1, \mathrm{~A}]$.

5.1.2.3. Progression of AKI has the worst outcome and at the same time resolution is associated with improved survival. Both persistence and new development of AKI are associated with a high shortterm mortality in patients with ACLF [1, B].

5.1.2.4. Serum creatinine levels $1.1-1.5 \mathrm{mg} / \mathrm{dl}$ or AKIN $\mathrm{I}$ are also associated with significant mortality in ACLF patients (2, B).

5.1.3. Treatment of renal failure in ACLF.

5.1.3.1. All attempts should be made to prevent development of new AKI in patients with ACLF $[1, \mathrm{C}]$.

5.1.3.2. Patients with ACLF should be stratified based on the PIRO (Predisposition, Infection/Inflammation, Response, Organ failure) score for identifying patients at risk of AKI development $[1, \mathrm{~B}]$.

5.1.3.3. Targeting the components of PIRO, i.e., combating systemic inflammation with anti-inflammatory strategies (for instance intravenous albumin, $\mathrm{N}$-Acetylcysteine), bilirubin reduction, avoidance of nephrotoxic drugs, aggressive management of circulatory failure and maintaining a high mean 
arterial pressure may prevent AKI development and progression in patients with ACLF [2, C].

5.1.3.4. Data on use of vasoconstrictors for AKI in ACLF are limited. Terlipressin given as an infusion is superior to noradrenaline in the management of HRS-AKI in patients with ACLF (B1). Terlipressin use in ACLF should be carefully monitored for adverse effects $(1, \mathrm{~A})$.

5.1.3.5. Severity of AKI, MELD score and ACLF grade predicts therapeutic response to terlipressin and albumin in patients with $\operatorname{HRS}-A K I$ in $\operatorname{ACLF}(1, B)$.

5.1.3.6. New treatments should be explored for patients with AKI-ACLF looking at systemic inflammation as a potential target $(2, \mathrm{~A})$.

5.1.3.7. Patients with ACLF with AKI persistence should be considered for additional pharmacologic interventions to prevent AKI progression and enhance AKI resolution $[2, \mathrm{C}]$.

5.1.3.8. Initiate RRT emergently when life-threatening changes in volume overload, hyperkalemia, hypernatremia and worsening metabolic acidosis not responding to conservative management $(1, \mathrm{C})$. The threshold for initiating RRT should be lowered when AKI occurs as part of multi-organ failure (1, $\mathrm{C})$, or in non-oliguric patients if the daily fluid balance cannot be maintained.

5.1.3.9. Biomarkers of tubular damage, urine NGAL and IL-18 need to be evaluated for a role in patients with ACLF to determine the need for early RRT or artificial liver support $(2, \mathrm{C})$.

\section{Hepatic encephalopathy}

The development of HE within 4 weeks of onset of jaundice is part of the criteria for defining acute-on-chronic liver failure (ACLF) [1, 2]. In the recent AARC data, HE was seen to be present in about $40 \%$ of the patients. Multiple prospective and retrospective studies had shown that HE in ACLF patients is associated with higher mortality, especially in those with grade III-IV encephalopathy, similar to that of acute liver failure (ALF). The experts proposed and defined cerebral dysfunction and cerebral failure as the presence of grade I and II HE and Grade III and IV HE, respectively. The presence of HE itself increases short-term mortality in ACLF as is the severity, i.e., grade of HE [3].

The pathophysiology of HE is complex, and impairment of brain energy and development of brain edema appear to be central in the pathogenesis of encephalopathy [117, 118]. Inflammation plays a greater role in the pathogenesis of HE in patients with ACLF than in patients without ACLF and is associated with a cytokine storm. Recent data also suggest that neuro-inflammation may have a significant role in brain disturbance [117]. Cerebral edema has been observed in ACLF, and even low cerebral edema can be detected by proper CT and MRI. Vasogenic cerebral edema as detected by advanced MRI techniques like magnetization transfer ratio (MTR), diffusion tensor imaging (DTI) and diffusion weighted imaging (DWI) is common, but rarely requires specific treatment [118]. Using advanced MRI techniques, Gupta et al. demonstrated presence of cerebral edema increases with severity of ACLF. Correlation between mean diffusivity (MD) values and IL-6 levels suggest pathogenic role of inflammation in cerebral edema. MELD score and MD values in frontal white matter have prognostic significance in ACLF [118]. Ammonia, systemic inflammation and oxidative stress are key factors in the pathogenesis of HE, which may be modulated by glutaminase gene alteration or by the presence of spontaneous shunts [118].

Management of HE in hospitalized patients requires admission to the ICU and includes-(1) identification and treatment of precipitating factors, including infections and (2) specific measures for decreasing hyperammonemia and systemic inflammation. High volume plasmapheresis or albumin dialysis and identification and embolization of portosystemic shunts may be required in refractory patients. Recently analyzed AARC data showed that ammonia was significantly and persistently high in patients with grade III and IV HE $(p<0.001)$. L-Ornithine L-Aspartate (LOLA) for Hepatic Encephalopathy has a conflicting role as far as $\mathrm{HE}$ and ammonia reduction in patients of cirrhosis is concerned. Few recent meta-analysis showed a positive role of LOLA in reduction of ammonia as well as improvement in encephalopathy. Hence, LOLA can be considered as a potentially beneficial therapy for ACLF patients with HE and/ or hyperammonemia [119]. However, a large prospective study would be needed. Emerging therapies include therapy for circulatory dysfunction and correction of hyponatremia [120].

Ammonia is a simple surrogate marker for HE in ACLF and correlates with severity of $\mathrm{HE} /$ cerebral failure. In the large AARC database, arterial ammonia levels were analyzed with respect to disease severity and outcome. At baseline, ammonia was significantly high in patients with cerebral dysfunction (HE grade I-II) and cerebral failure (HE grade III-IV). The patients who showed improvement in HE grades at day 4 and day 7 showed significant reduction in plasma ammonia level [121]. Dynamic change in ammonia level correlates well with clinical course of HE. However, ammonia-targeted therapy needs further trials and validation.

\section{Recommendations}

\subsection{Hepatic encephalopathy in ACLF.}


5.2.1. HE, including grade 1-2 HE (organ dysfunction) and grade 3-4 HE (organ failure), is present in about a third of the ACLF patients (2, B).

5.2.2. HE with all grades of severity and independent of other organ failures is associated with increased mortality; the mortality is higher in grade $3-4$ (organ failure) compared with grade 1-2 HE (organ dysfunction) [1, B].

5.2.3. Inflammation plays a major role in the pathogenesis of HE in patients with ACLF and is associated with cytokine storm [1, B].

5.2.4. Management of HE in hospitalized patients requires admission to the high dependency or intensive care unit and includes-(1) identification and treatment of precipitating factors, including infections, and (2) specific measures for decreasing hyperammonemia and systemic inflammation. Large volume plasmapheresis or albumin dialysis and identification and embolization of portosystemic shunts may be required in refractory patients $[1, \mathrm{C}]$.

5.2.5. Ammonia is a simple surrogate marker for $\mathrm{HE}$ in ACLF and correlates with severity of HE/cerebral failure [2, B].

5.2.6. Dynamic change in ammonia level correlates well with clinical course of $\mathrm{HE}$ [2, B].

5.2.7. Ammonia-targeted therapy needs further trials and validation $[2, \mathrm{~B}]$.

5.2.8. Lactulose, rifaximin, NH3-lowering strategies remain the main therapy for $\mathrm{HE}$ in patients with cirrhosis (1, B).

\section{Coagulation in ACLF}

In ACLF, alterations in primary and secondary hemostasis result in rebalanced coagulation, which leads to either bleeding or thrombotic episodes [122-125]. In addition, organ failures in ACLF may further disturb cirrhotic hemostatic imbalance. These include circulatory dysfunction [123], systemic inflammatory response syndrome [124, 125], sepsis, endogenous heparin-like substances or heparinoids [126] and renal dysfunction [127, 128].

Coagulation system in liver diseases is usually assessed by INR and platelet counts. Study by Premkumar et al. [129] and Blasi et al. [130] suggests that the ACLF is a hypocoagulable and hypofibrinolytic disorder and development of SIRS $[131,132]$ and sepsis further increases the hypocoagulability in these patients with increased chances of coagulopathic bleeding. Indirect evidence suggests that the endogenous heparinoids in ACLF patients with SIRS and sepsis could induce increased hypocoagulable state [130]. Conventional tests are insensitive to assess the complex coagulopathy in ACLF can be further complicated by SIRS and sepsis. The development of SIRS by day 7 further increases the INR $(p<0.001)$; however, no effect on platelets has been noted. ACLF cases with sepsis at presentation show increased INR and low platelets. Studies indicate the decline of platelets in first week associated with increased chances of organ failure and short-term mortality [131]. Acute variceal bleed at the time of presentation did not have association with baseline platelet counts; however, the INR was significantly higher in bleeders than non-bleeders in the recently analyzed AARC data.

PT-INR or prothrombin times is useful for prognostication, but are insensitive for detection of coagulopathy. More evidence-based algorithmic approach is needed for diagnosis and management of ACLF-induced coagulopathy.

\section{Recommendations}

\subsection{Coagulation in ACLF.}

5.3.1. ACLF is a hypocoagulable state and this can get escalated with the development of SIRS and sepsis $[2, \mathrm{C}]$.

5.3.2. Traditional coagulation measures, including prothrombin time (PT), activated partial thromboplastin time (aPTT), international normalized ratio (INR), fibrinogen levels and bleeding time (BT) do not measure bleeding risk in ACLF [2, B].

5.3.3. Coagulopathy assessment and management in ACLF should be guided based on global coagulation assessment system [ROTEM/TEG/SONOCLOT] $[2, \mathrm{C}]$.

5.3.4. Patients need to be characterized as bleeding or thrombosis phenotype by clinical assessment of major bleeding and D-dimer assay, respectively $[2, \mathrm{C}]$.

5.3.5. Global viscoelastic tests (TEG/Sonoclot/ ROTEM) provide a more physiologic assessment of coagulation and should be considered to guide blood transfusion requirements in liver transplantation $[1 \mathrm{~A}]$ and other major surgery [2B] and invasive procedures [2C]. Application of global viscoelastic testing with ACLF in the ICU setting requires more data $[2 \mathrm{C}]$.

\section{Portal and systemic hemodynamics in ACLF and variceal bleed}

Portal hypertension in liver disease is associated with both structural damage, which is the irreversible component, and dynamic component that includes increase in cytokine production, endothelial dysfunction, impaired vasorelaxation, and impaired vascular relaxation, which may be reversible component in the pathophysiology of portal hypertension after recovery. Thus, increased portal pressure in ACLF not only contributes to variceal bleeding but also to development 
of rapid onset ascites and other systemic complications including organ failures of ACLF. Majority of the patients with ACLF die during the first 45 days (median time to death 15 days) since the diagnosis [132]. ACLF patients have been shown to have much higher HVPG as compared to compensated cirrhotics. Among survivors of ACLF, complications such as ascites, coagulopathy gradually regress by 3 months [133]. It is thus likely that in patients with ACLF, the portal pressure gets acutely elevated and, after recovery, hepatic inflammation and cytokine levels decrease, which leads to improvement in hepatic as well as systemic hemodynamics. This was also indicated in a recent Asia Pacific multi-center study that higher cardiac output correlated with 30-day mortality $(p<0.019)$ and higher HVPG was associated with increased risk of variceal hemorrhage and mortality at $30(p<0.02)$ and 90 days $(p<0.001)$ [133]. Notably, these features were more pronounced in alcoholic hepatitis patients [134]. Choudhury et al. showed that baseline HVPG and mean pulmonary artery pressure were independent predictors of three-month mortality in ACLF [135].

\section{Variceal progression and role of pharmacotherapy} in ACLF The rapid development of varices and bleeding is a matter of great concern in ACLF patients. It is important to determine the need of acute portal pressure reduction in these patients. It is likely that the patients would benefit from portal hypertension-reducing drugs like beta-blockers, especially in the acute phase of portal hypertension. NSBB have beneficial effects on the severity of portal hypertension, which requires both the beta- 1 and beta- 2 actions of the drug to ameliorate splanchnic vasodilation and high cardiac output.

Patients on NSBB had less severe grade of ACLF and a slower progression of ACLF during the study period. Patients who were receiving NSBBs in the previous 3 months and discontinued NSBBs $(n=78)$ after development of ACLF had a higher mortality (37\% vs. $13 \%$ ) and the main difference between those who discontinued or did not discontinue $\mathrm{BB}$ was the presence of circulatory dysfunction (hypotension requiring pressers) and respiratory failure. In another RCT comparing carvedilol with placebo in patients with ACLF (defined by APASL criteria) with either no or small esophageal varices and no contraindication to carvedilol use, carvedilol was found to reduce mortality, development of SBP and AKI at week 4 [135]. Thus, it is clear that patients should be continued on NSBBs, if feasible, even if ACLF develops [136].

Regarding the safety and radiation exposure of technical procedures for hepatic hemodynamics, Hari et al. studied the safety profile of HVPG measurement prospectively [137]. Accordingly, HVPG procedure showed a good safety profile and the radiation exposure was in most of the cases low. However, the HVPG measurement is invasive and difficult for routine clinical practice. Therefore, non-invasive surrogates that correlate well with invasive HVPG measurements are urgently needed in patients with ACLF [137-139].

PICD incidence, presentation, diagnosis and management in ACLF Ascites is one of the syndrome defining components and in the AARC study, about $91 \%$ patients had ascites at presentation. About one-third of the patients presenting with ACLF do require paracentesis for severe grade ascites [140]. The presence of ascites in ACLF is different in many aspects from decompensated cirrhosis or AD [141]. Development of PICD in ACLF is associated with very high mortality. Albumin infusion was shown to significantly reduce mortality. Albumin infusion also helped in reducing to nearly half the incidence of development of new complications such as hyponatremia, hepatic encephalopathy and acute kidney injury. A high PRA level in ACLF patient reflects state of severe systemic inflammation, high portal pressure and systemic circulatory dysfunction [142, 143].

All precautions and monitoring including plasma renin activity are needed in undertaking ascetic tap in ACLF patients. Ascites in ACLF is part of acute portal hypertension and large volume paracentesis significantly alters the hemodynamics. Pharmacological agents, such as use of vasoconstrictors, should be studied to reduce the incidence of PICD in ACLF patients.

\section{Recommendations}

5.4.1. Systemic, hepatic and pulmonary hemodynamics in ACLF.

5.4.1.1. Baseline HVPG is an important predictor of mortality in $\operatorname{ACLF}(2, \mathrm{~B})$.

5.4.1.2. The reduction in HVPG significantly influences the management of $\operatorname{ACLF}(2, \mathrm{C})$.

5.4.1.3. The safety and standardized procedure of HVPG measurement should be emphasized. Non-invasive surrogates of HVPG need to be investigated in $\operatorname{ACLF}(1, \mathrm{C})$.

5.4.2. Variceal progression in ACLF and role of preemptive $B B$ therapy.

5.4.2.1. Increased portal pressure in ACLF can not only contribute to variceal bleeding but also other systemic complications and organ failures in ACLF $[1, \mathrm{~A}]$.

5.4.2.2. NSBBs may reduce systemic inflammation and may have beneficial effects in ACLF patients over and above their portal hemodynamic effects [2, A].

5.4.2.3. ACLF patients should be started or continued on NSBBs, if there are no contraindications [2, B].

5.4.3. PICD incidence, presentation, diagnosis and management. 
5.4.3.1. PICD is a result of significant derangement of systemic and splanchnic hemodynamics with a decrease in effective arterial blood volume, which is triggered by large volume paracentesis $(>5 \mathrm{~L})[1, \mathrm{~A}]$.

5.4.3.2. PICD occurs in about $80 \%$ of ACLF patients when a large volume paracentesis is performed without additional therapeutic management. However, the incidence is reduced when volume expansion with albumin is used $[1, \mathrm{~A}]$.

5.4.3.3. Terlipressin, a vasopressin analog, is partially effective and has been shown to have a synergistic effect with albumin in preventing PICD $[1, \mathrm{~B}]$.

5.4.3.4. Non-selective $\beta$-blockers, such as propranolol, have been suggested to increase the risk of PICD and mortality in cirrhotic patients with refractory ascites. There is lack of data in patients with ACLF [2, B].

\section{Prognostic models and disease severity scores for ACLF}

\section{Prognostic models and disease severity scores}

The disease severity assessment is needed for prognostication and to guide the therapy [144-146]. Disease severity scores such as model for end-stage liver disease (MELD) have been considered for organ allocation. However, MELD score does not take into account cerebral, circulatory and/or respiratory failures, thus giving no priority to patients with ACLF [147]. The various ICU scores like Sequential Organ Failure Assessment (SOFA), Acute Physiology and Chronic Health Evaluation (APACHE II) have also been evaluated for ACLF patients [147]. A study by Garg et al. in 2012 from New Delhi showed the predictability of these scores and also the relevance of one, two or more organ failures [28]. Subsequently, CLIF consortium has developed CLIF-SOFA score for assessing disease severity and prognostication in ACLF [11]. We had earlier shown that patients of ACLF have a high mortality in the presence of $\mathrm{HE}$ and hyponatremia in addition to high MELD, APACHE II and SOFA scores [28], necessitating inclusion of these parameters. A clinical event like HE or the laboratory parameters like bilirubin, creatinine, INR, serum sodium, plasma lactate or the liver histopathology reports and various disease severity scores do give near accurate prognostication.

Furthermore, the available prediction scores have been validated at baseline, but none has been evaluated in a dynamic manner for prognostication in ACLF patients. The severity of ACLF, rapid progression, development of sepsis and subsequent multi-organ failure (MOF), poor outcome with liver transplantation at the onset of MOF needs dynamic monitoring. Recent studies support for developing a dynamic model that could predict the outcome and appropriate time for LT. Chan et al. [147] in 149 patients showed that APACHE II scores $\geq 12$ and MELD scores $\geq 28$ after the first week of treatment were independent predictors of mortality. Mathurion et al. [148] showed that in the absence of response to corticosteroid for AH as assessed by Lille score on day 7 and consideration of early LT lead to significant cumulative 6 -month survival rate $(77 \pm 8 \%$ vs. $23 \pm 8 \%$, $p<0.001)$. In large UK and US cohort of severe autoimmune hepatitis showed that not the baseline MELD/UKELD but the use of corticosteroid and no improvement in MELD/ UKELD scores within 7 days had a poor outcome and suggested early consideration of other strategies including liver transplant [149-152]. A dynamic model that could predict the reversibility or need for liver transplant is urgently required. Early prediction of transplant-free survival, decision for transplant before onset of sepsis or multi-organ failure and prioritization for liver transplant could help improve the outcome of these patients.

\section{Organ dysfunction and organ failure in ACLF for early risk stratification}

The CLIF-SOFA and the CLIF-OF (Organ Failure) scoring and the cutoff were developed arbitrarily and included patients with hepatic and non-hepatic insults [11]. The organ failure was solely derived based on a consensus opinion by the experts [11]. The score is a bit cumbersome and becomes predictive of mortality only when extrahepatic organ failures are included. Earlier studies [28] and a recent study [152] showed that a simple score considering only the number of organ failures is easier to recall in predicting mortality in ACLF patients. The recently established and evaluated TPPM model in HBV ACLF patients from a single center large cohort, which displayed a superior predictive ability when compared with MELD and MELD-Na models [n-153]. TPPMs used TBIL, INR, HBV DNA and complications as parameters [153, 154]. Based on current multinational cohort, TPPMs demonstrated superiority to CLIF-C OFs, CLIF-C ACLFs, MELDs and MELD-Na in predicting 28-day and 90-day mortality in cirrhotic HBVACLF patients [155].

The AARC score, as mentioned above, provides a physician a window to decide early and explore definitive therapies including liver transplantation (Table 4). A patient in whom the AARC score increases from 5 or 6 to 11 and above (change in grade of liver failure from I to III) at day 4 and day 7 increases the mortality significantly and a need for emergent transplantation in patients who fulfill the criteria. At the same time, the persistence of grade I or II liver failure till day 7 predicts improved survival, and a possibility of conservative treatment to be effective [3] (Fig. 4). AARC score has been compared with other 
scores for assessing the severity of ACLF such as SOFA, CLIF-SOFA, and MELD. The AARC score has been found to be superior to the other scores [3] (Fig. 5).

\section{Recommendations}

\subsection{Prognostic models and disease severity scores} for ACLF.

6.1. AARC score and other prognostic models.

6.1.1. AARC score is a very good prognostic model for the severity assessment and has been adequately validated. It has been proven to be superior to MELD/MELD Na., CLIF-SOFA and SOFA scores for patients with ACLF [2, A].

6.1.2. The cumulative mortality increases with rise in AARC score in the first week in Grade I (5-7), II (8-10) and III (11-15), respectively [2, A].

6.1.3. Treatment recommendations for ACLF should be based on AARC score. A trend of AARC score within first week can predict the need of liver transplant. Score of $<10$ at presentation or decrease in score below 10 by the end of first week is associated with higher chance of survival $[1, \mathrm{~B}]$.

6.1.4. Patients with AARC Score $>10$ should be listed for LT. Transplant evaluation based on the AARC score needs prospective studies [2, B].

6.1.5. AARC-ACLF score be estimated at admission in patients diagnosed to have ACLF as per AARCACLF definition [1, B].

6.1.6. AARC-ACLF score should also be estimated on Day 4 and Day 7 to predict the course of illness and prognosis $[2, \mathrm{~A}]$.

6.1.7. The TPPM model has a superior predictive value for HBV ACLF outcomes than MELD and CLIFFSOFA models $(2, \mathrm{C})$.
6.1.8. AARC score holds good in predicting the outcome in critically ill ACLF patients $[1, \mathrm{C}]$ ).

6.1.9. Lactate should be used for defining the severity of the ACLF syndrome. Lactate clearance compared to baseline lactate is a better indicator of patient status $[1, \mathrm{C}]$.

6.1.10. There is limited role of Renal Replacement Therapy (RRT) \& Extra Corporeal Liver Support System (ECLS) to treat hyperlactetemia [2, B].

6.2. Organ dysfunction and organ failure in ACLF for early risk stratification.

6.2.1. Organ dysfunction and failure should not be used in the definition, but for prognostication in patients with ACLF [1, A].

6.2.2. Liver failure should be defined by a combination of bilirubin and INR and should be classified as mild/ moderate and severe based on AARC as all patients have liver failure $[1, \mathrm{~B}]$.

6.2.3. Liver, kidney and brain remain organs of utility in patients with ACLF [1, B].

6.2.4. Organ dysfunction and failure for brain should be based on AARC score $[1, \mathrm{C}]$.

6.2.4. Respiratory and circulatory failure may be considered as organs of futility in patients with ACLF which may contraindicate liver transplant $[1, \mathrm{C}]$.

6.2.5. For the diagnosis of organ dysfunction for kidneys in patients with ACLF as for patients with cirrhosis, AKIN criteria should be used [1, A].

6.2.6. Kidney failure should be defined based on serum creatinine above $1.5 \mathrm{mg} / \mathrm{dl}$ as per the AARC score $[2, \mathrm{~B}]$.

6.2.7. The mortality in ACLF increases with the number of organ failures $(1, \mathrm{C})$.

Table 4 AARC score and ACLF grade

\begin{tabular}{|c|c|c|c|c|c|}
\hline \multicolumn{6}{|c|}{ AARC score } \\
\hline Points & Total Bilirubin (mg/dl) & HE Grade & PT-INR & Lactate $(\mathrm{mmol} / \mathrm{l})$ & Creatinine $(\mathrm{mg} / \mathrm{dl})$ \\
\hline 1 & $<15$ & 0 & $<1.8$ & $<1.5$ & $<0.7$ \\
\hline 2 & $15-25$ & I-II & $1.8-2.5$ & $1.5-2.5$ & $0.7-1.5$ \\
\hline 3 & $>25$ & III-IV & $>2.50$ & $>2.5$ & $>1.5$ \\
\hline \multicolumn{6}{|c|}{ Minimum-5, Maximum-15 } \\
\hline \multicolumn{6}{|c|}{ AARC-ACLF grade } \\
\hline \multicolumn{5}{|l|}{ Grade } & Score \\
\hline \multicolumn{5}{|l|}{ I } & $5-7$ \\
\hline \multicolumn{5}{|l|}{ II } & $8-10$ \\
\hline \multicolumn{5}{|l|}{ III } & $11-15$ \\
\hline
\end{tabular}




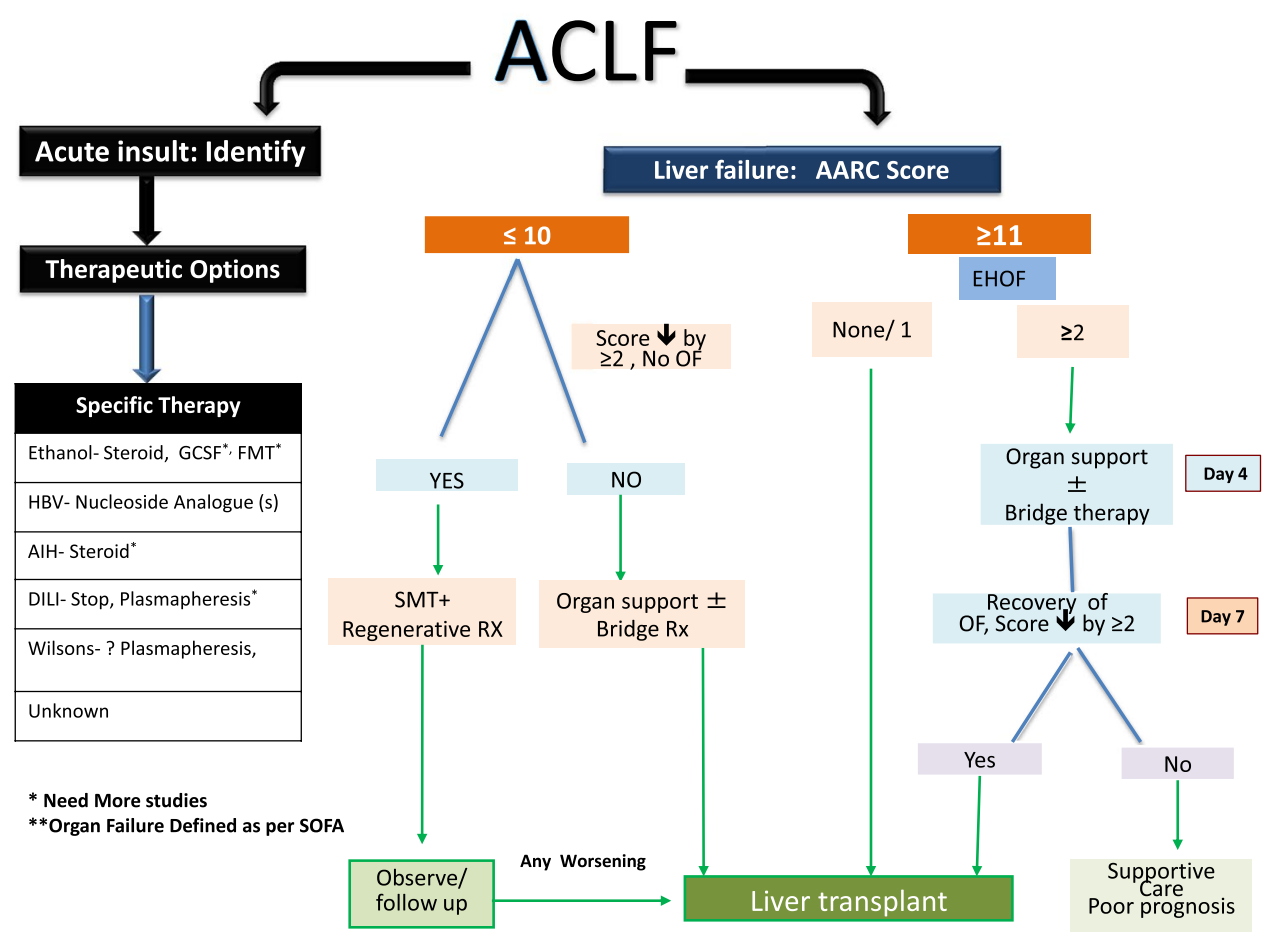

Fig. 4 Algorithm for management of ACLF. The algorithmic approach to ACLF is highlighted based on the severity of liver failure, acute etiology and specific therapy and dynamic disease course. The specific treatment initiated, but if the disease severity is more, i.e., AARC Score (consideration of bilirubin, creatinine, INR, lactate and HE grade) 11 or more the response is poor with best medical supportive car; hence, early consideration for liver transplant should be

\section{Treatment of ACLF}

\section{Antiviral strategies in ACLF HBV reactivation}

The presence of high HBV DNA [>10(5) copies/ml/ or $>2 \times 10(4) \mathrm{IU} / \mathrm{ml}]$ is highly sensitive and specific for the diagnosis [152]. Early and rapid reduction of HBV DNA is the essence of therapy [153]. Several studies have indicated that if the reduction in DNA of $>2 \operatorname{logs}$ could be achieved within 2 weeks, the survival could be improved. This could be related to suppression of hepatocellular necrosis and cytokine release [154].

Besides patients who present with ACLF, it is worthwhile that prophylactic therapy should be considered for HBsAg-positive patients undergoing chemotherapy [155]. There are insufficient data to recommend antiviral therapy for HBsAg-negative and anti-HBc-positive patients with possible reactivation of occult $\mathrm{HBV}$ infection [156, 157].

\section{Recommendations}

7.1. Antiviral strategies in ACLF HBV reactivation. done, whereas other group needs to be seen for 4-7 days with specific therapy and standard medical therapy. Any deterioration or AARC score 11 or more needs to consider LT. The presence of extrahepatic organ failure needs to be managed, and optimization and improvement need to be correlated with over all recovery else poor prognoses to be considered

7.1.1 Nucleos(t)ide analogs should be started immediately in all $\mathrm{HBV}$-infected patients at presentation while waiting for confirmation by HBV DNA level. Potent antiviral drugs, such as tenofovir, tenofovir alafenamide or entecavir, should be used [2,B].

7.1.2. Assessment of reduction of HBV DNA level at day 15 after nucleos(t)ide analogs is encouraged; if $<2$ $\log$ reduction, it suggests poor prognosis $(2, \mathrm{~B})$.

\section{Liver transplantation}

A characteristic feature of ACLF is its rapid progression, the requirement for multiple organ support and a high incidence of short- and medium-term mortality of 50-90\%. The 28-day mortality rate was 15 times higher in patients with ACLF as compared to other chronic liver disease (CLD) patients [1-11, 158]. Patients with ACLF are susceptible to infection and early transplant-free survival is very low [159, 160]. Patients who develop infectious complications (particularly pneumonia and/or sepsis) and patients who receive renal 


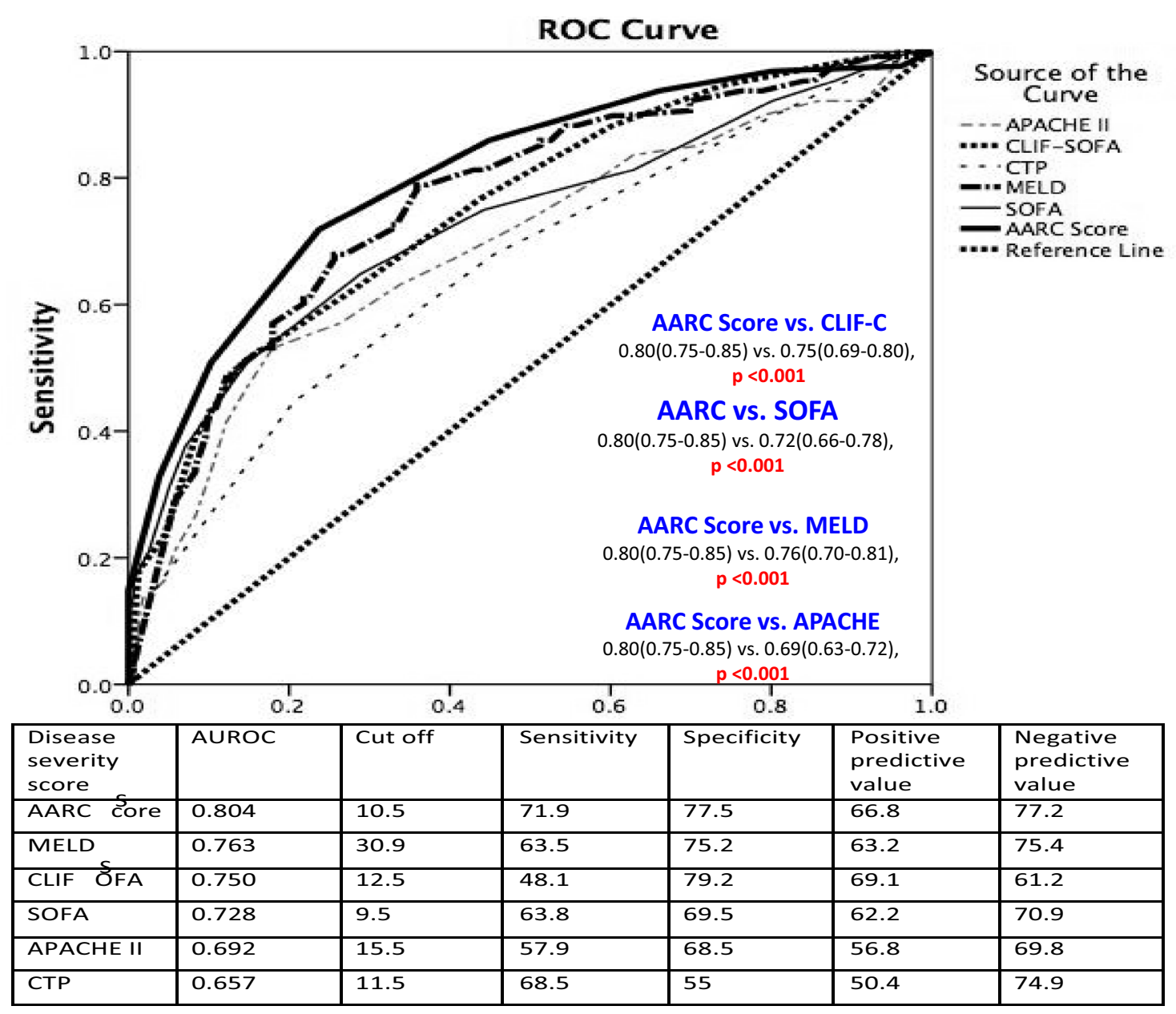

Fig. 5 Comparison of AARC score against other disease severity score

replacement therapy or mechanical ventilation are less likely to undergo liver transplantation. Established sepsis/MODS precludes transplant and is associated with poor outcome. Both deceased and living donor transplants are viable and very useful options with very good results [161]. Liver transplant survival has been shown to be above $90 \%$ in patients with HBV reactivation [162].

Most important aspect in managing this group of sick patients is to decide the time frame and an algorithm. If the patient is too sick from the beginning and needs early LT without waiting for spontaneous recovery, this should be decided soon enough. On the other hand, patients who are salvageable and need time for recovery should be monitored closely in a time frame before deterioration so that they either recover or can undergo LT at an appropriate time. The third group is of those who need bridge therapy observed while on supportive care and bridging therapy, to define nonresponse and emergency LT or for transplant-free survival.
Every ACLF patient at admission needs to be assessed for disease severity score, presence of SIRS with or without sepsis, HE and number of organ dysfunction/failure. The baseline MELD > 28, AARC Score $>10$, advanced HE in the absence of overt sepsis or multi-organ failure can be considered for early LT [3]. In the absence of LT option, these patients can be offered early bridge therapies in the form of therapeutic plasma exchange and liver dialysis and the response could be evaluated by end of first week and the possibility of being listed for LT or awaiting spontaneous recovery $[3,163]$. The maximum recovery from organ failure, resolution of sepsis and eligibility for LT was observed in the first week [3]. The SIRS and/or sepsis and development of AKI occur by days 7-15; hence, the interventions like prophylactic antibiotics, periodic septic screening with the help of biomarkers and avoidance of nephrotixic drugs were needed [77].

ACLF is characterized by rapid downhill course with extrahepatic organ failure and high short- and 
medium-term mortality ranging from 34 to $51 \%$ [1]. Although many prediction models of early transplantation listing exist, none reliably predicts chances of reversibility of ACLF. A recent study showed that the ACLF patients develop SIRS and sepsis within 7 days of hospitalization [77]. Lin et al. [164] and Pamecha et al. [165] proposed serial assessment of these groups of patients in the first week of hospitalization for prioritization for liver transplantation. However, being sick, often critically ill and admitted to ICUs, rapid progression of liver failure and onset of multi-organ failure, transplantation was feasible in $~ 25 \%$ of patients [165]. Recently, a study showed that the LT waiting list mortality in ACLF patients is around $67 \%$ and is predominantly due to sepsis, respiratory failure with mechanical ventilation, high vasopressor requirement and need of RRT [165]. Though objective criteria were not used to define eligibility for LT, patient characteristics that were reported to consider an ACLF patient unfit were (1) sepsis with 2 or more organ failures or uncontrolled sepsis [166], (2) advanced azotemia, i.e., serum creatinine $>4 \mathrm{mg} / \mathrm{dl}$ or increase in creatinine by $300 \%$ from baseline or the need of Renal Replacement of therapy [167], (3) Respiratory failure [severe ARDS defined by a $P /$ F ratio $<150]$ or HE requiring ventilator support $>72 \mathrm{~h}$ [168] (4) 4 or more organ failures anytime, (5) active gastrointestinal bleeding, and/or (6) hemodynamic instability requiring $>3 \mathrm{mg} / \mathrm{h}$ noradrenaline [169].

On the other hand, in the absence of liver transplantation, the outcome is dismal. In fact, liver transplant is potentially the only curative treatment option with good outcome, irrespective of etiology in this cohort. An analysis of 1021 patients from AARC cohort suggested MELD or MELD with HE is a good prediction model for making decision for LT. A patient with MELD $>27$ needs listing, a score of 30 or above with presence of encephalopathy or new onset $\mathrm{HE}$, bilirubin of $>22 \mathrm{mg} / \mathrm{dl}$ with INR $>2.5$ and grade III-IV $\mathrm{HE}$ is associated with $80 \%$ mortality within 28 days and persistence of the same till day 4 is associated with mortality close to $100 \%$. Hence, these patients need emergent LT either at baseline or upon no improvement within day 4-7 of hospitalization [163]. So first week of presentation in ACLF is crucial $[77,170]$. This concept is supported by a window of 1 to 2 weeks, i.e., being sick and with no improvement by supportive care [171].

Disease severity scores such as MELD have been considered to determine organ allocation. This disease severity score has not taken into consideration of cerebral, circulatory and respiratory failure and does not offer any priority despite being sick $[172,173]$. The recently published dynamic AARC model consisting of bilirubin, creatinine, INR, grade of hepatic encephalopathy and plasma lactate is a reliable model to predict the outcome at each time point, hence, the need of LT can be considered for prioritizing
LT but further studies needed on this [3]. Emergency LT, promotion of live donor transplant and allocation priority in deceased donor setting needs consensus and further large size studies [77, 163, 170-176].

Sometimes, patients with ACLF have rapidly worsening liver and renal functions, needing quick decisions based on renal dysfunction. There is paucity of data on simultaneous liver and kidney transplant in this subset of ACLF patients [112].

\section{Recommendations}

7.2. Liver transplantation.

7.2.1. No validated criteria and scoring system for early and correct identification of patients with ACLF who would benefit from early liver transplantation $[2, \mathrm{~A}]$.

7.2.2. LT should be offered early in the course of ACLF. When indicated, early liver transplantation avoids complications of sepsis and multiorgan failure [1, B].

7.2.3. SIRS, sepsis, HE, liver failure, extrahepatic organ dysfunction/organ failure are important prognostic factors $[2, \mathrm{~A}]$.

7.2.4. Organ failure per se should not be a contraindication for liver transplantation, except if cardiac or pulmonary support is needed or there is rapidly progressive organ failure at day 4 or $7[2, \mathrm{C}]$.

7.2.5. APASL AARC model seems to be better in patient selection for liver transplantation as it enrolls patients early enough, before organ failure. However, the AARC score needs to be validated in large and varied populations and also its capability to predict post LT outcome [2, B].

7.2.6. Patients with HBV reactivation with intermediate MELD should be assessed for early transplant if cirrhosis, bilirubin $>10 \mathrm{mg} / \mathrm{dL}, \mathrm{PT}<40 \%$ and platelet $<100 \times 10^{9} / \mathrm{L}[2, \mathrm{C}]$.

7.2.7. Steroid ineligible patients with severe alcoholic hepatitis should be listed on priority for liver transplant. Selective use of liver transplantation can be lifesaving for medically refractory alcoholic hepatitis $[1, \mathrm{~A}]$.

7.2.8. Liver transplantation should be reserved for severe alcoholic hepatitis patients with low risk of recidivism as assessed by a formal psychosocial evaluation $[1, \mathrm{~A}]$.

7.2.9. Transplant evaluation can be started based on liver specific dynamic scores by the end of first week of medical treatment or earlier [2, B].

7.2.10. Allocation of cadaveric livers should depend on maturity of cadaveric program in the country $[2$, C]. 
7.2.11. Patients with advanced ACLF also have good outcome after LT [1, A].

\section{Liver dialysis and emerging therapies in ACLF}

The hepatocellular injury in ACLF is driven to a large extent by a "cytokine burst", with elevated levels of multitude of cytokines, small molecular weight toxins, and vasoactive substances which are known to accumulate secondary to the failing liver [122]. There is an additional challenge of the injury due to endotoxin and metabolites released from gut bacteria. These toxins not only potentiate the hepatic injury but also deprive the liver of an environment, which is conducive for regeneration. The released toxins are responsible for the systemic inflammation, loss of adaptive and innate immunity and cause vital organ dysfunction, which affects all the major organs [174].

Extracorporeal liver support therapies are used to bridge the liver until recovery or liver transplantation in patients with ALF and ACLF. Various randomized controlled trials in patients with ACLF have shown improvement in HE, hepatorenal syndrome, circulatory dysfunction and immune dysfunction without improvement in transplant-free survival [175-182]. In the most recent meta-analysis and systematic review, no benefit of MARS treatment in reducing mortality as compared to SMT was noted [182]. Even though both these meta-analysis have the limitations of enrolling a heterogenous group of patients. However, contrary results were shown by systematic review by Kjaergard et al. where it was seen that ALS reduced mortality by $33 \%$ in patients with ACLF as compared to SMT [183, 184]. More recently, studies have shown that ALS could be an effective form of bridging therapy in patients with ACLF with high MELD scores awaiting liver transplantation and many believe that it is a futile exercise in the absence of liver transplant [185, 186]. These results have been substantiated by the recently published two large European randomized multicentric controlled trials, i.e., HELIOS (for Prometheus) [177] and RELIEF trial (for MARS) [176] which failed to show any benefit with these modalities on short-term transplant-free survival which was the primary end point of these studies. The foremost reason for no demonstrable survival benefit with the currently available artificial liver support systems is the functional incompetence as most of these provide only the detoxification function of the entire armamentarium of liver functions and thus incorporation of synthetic function by living hepatocytes, i.e., the "bioartificial liver" or therapies to potentiate hepatic regeneration look more realistic. The other major challenge that remains is to decide the timing of therapy so as to derive maximal therapeutic benefit, i.e., whether to consider it before or after the onset of sepsis because by the time multi-organ failure is manifest, the benefits of intervention with these devices are not to be expected.
In a large proportion of patients with ACLF, however, liver transplant is not feasible, due to lack of an organ, a donor, severity of the illness or other social challenges. There are few alternatives at present to liver transplant. There have been promising results of the use of growth factors in such patients. Garg et al. [28] have shown that granulocyte colony-stimulating factor (GCSF) can help in hepatic regeneration by mobilizing bone marrow-derived CD34 + cells. In addition, it significantly reduced the development of sepsis and subsequent multi-organ failure. These data were substantiated in another study from the East in patients with HBV-related ACLF [187-194].

However, despite the encouraging results and two randomized controlled clinical trials, it was felt that the use of these agents should be undertaken only under protocols and more data are required before recommending routine use of these agents.

\section{Recommendations}

7.3.1. Plasma exchange appears to be a promising and effective bridging therapy in patients with ACLF to liver transplant or spontaneous regeneration [1, C]

7.3.2. Plasma exchange can be safely undertaken in patients with ACLF in specialized liver units [2, B].

7.3.3. Plasmapheresis may be considered as a specific therapy for patients with Wilson's disease and patients with severe flare of autoimmune liver disease (deemed unsuitable for steroids) [2, B].

7.3.4. Combination of $\mathrm{PE}$ with therapies to potentiate liver regeneration should be evaluated in patients with ACLF $[2, \mathrm{C}]$.

\section{Acute-on-chronic liver failure in children}

An extensive PubMed search using keywords 'Acute-onchronic liver failure in children; ACLF in children; Pediatric acute-on-chronic liver failure; Pediatric ACLF', returned only 5 studies, from 3 Indian centers [195-199]. The data on pediatric ACLF are limited and largely retrospective. Pediatric ACLF, although less commonly described in the literature, is not uncommon with a recent study demonstrating that around 14\% of all CLD presented as ACLF [195]. ACLF was the first manifestation of a previously unknown underlying CLD in 75-100\% cases as reported in some studies [196-198]. The combined data from the 3 centers showed that Wilson disease $(41.2-45.7 \%)$ followed by autoimmune liver disease (17.6-41.9\%) are the commonest CLD presenting as ACLF followed by cryptogenic cirrhosis (3.2-41.2\%) [195-199]. The acute precipitating event was a hepatotropic viral insult (37-94.1\%) in most. Flare of autoimmune liver disease (9.6-17\%) and Wilson disease (0-27\%), defined as 
ACLF in the absence of a recognizable acute event were also reported [196, 197], although the definition used is not established. In a study, no acute hepatic event was found in $29 \%$ of patients, a proportion of which could have been flare of Wilson disease [198]. As cholangitis is not currently accepted as parenchymal insult, which happens to be the most important event leading to decompensation in biliary atresia, the experts decided to exclude biliary atresia from ACLF definition.

Only $8.7 \%$ of the pediatric ACLF cohorts were $\leq 3$ years. Metabolic liver diseases (MLD) account for majority of CLD in this age group. On analysis of MLD data, it was found that only $3 / 63$ (4.8\%) could fulfill the definition of ACLF but were labeled as ALF as there is some overlap in the 2 definitions [1, 199]. Children with MLD also failed to fulfill the definition of ACLF as a proportion of them either had longer jaundice to HE/ascites interval [200] or had anicteric liver failure. The common acute precipitating events of pediatric ACLF present less often before 5 years of age: acute hepatitis A in 15.7\% (personal communication ILBS data) \& drug hepatotoxicity: 27.8\% [201].

\section{Do we need a modified definition of pediatric ACLF?}

There is no separate definition of pediatric ACLF. The major issues with the current definition in children are: (1) clinical identification of hepatic encephalopathy is often difficult and/or delayed specially those below 3 years of age, (2) clinical ascites may be difficult to identify especially in younger children with organomegaly, (3) some pediatric liver disease may present with liver failure without jaundice. The current ACLF definition requires jaundice to be followed by clinical ascites or HE within 4 weeks [1]. A delayed clinical identification or nonidentification of HE/ascites will lead to the patient either being identified beyond the period of golden therapeutic window or not even diagnosed as ACLF. To circumvent the issue of difficult identification of HE, pediatric acute liver failure study group has defined acute liver failure as an INR greater than 2 regardless of the presence or absence of clinical HE [199]. Pediatric ACLF cohort at Institute of Liver and Biliary Sciences (ILBS) was evaluated to determine if the cutoff INR for defining ACLF can be increased to 2 regardless of the presence or absence of clinical HE. In the presence of HE, poor outcome was seen in $42.9 \%$ and $56.6 \%$ of those with INR between 1.5 and 2 (18/90) and those with INR $\geq 2$ (74/90), respectively. As identification of $\mathrm{HE}$ is important but clinical identification often difficult, ammonia which has good correlation with HE [202] was evaluated as a surrogate marker. Ammonia performed poorly for diagnosis of HE with AUROC of 0.642 and an ammonia level more than $150 \mathrm{ug} / \mathrm{dl}$ diagnosed HE with $61.3 \%$ sensitivity and $70 \%$ specificity. EEG is another surrogate but is difficult to perform, not easily available and has not been standardized in children [203]. In the absence of a good surrogate, the experts agreed that clinical HE should continue to remain part of the definition. West Haven scale is used in older children, whereas modified HE assessment scale can be used for identifying and grading HE in children up to 3 years of age [199]. Detection of clinical ascites is difficult or delays diagnosis. Radiological ascites (mild to massive) from the ultrasound report and clinical ascites from the discharge summaries were compared in 127 children aged up to 3 years with CLD. The sensitivity of clinical examination to detect ascites was $71 \%$ with $29 \%$ being missed or identified later when the ascites increased further. Hence, the experts agreed to replace clinical ascites with clinical/radiological ascites in children.

\section{Outcome and prognostication in pediatric ACLF}

Theoretically, pediatric ACLF should have a better outcome than adults as the 2 commonest CLD, i.e., Wilson disease and autoimmune liver disease presenting as pediatric ACLF have specific medical therapy, better hepatic reserve (due to shorter duration of illness) and lesser incidence of comorbidities. Outcome has been defined at different time points in the published pediatric literature [195-204]. Wilson disease and cryptogenic cirrhosis have poor prognosis with $48.8 \%$ and $30 \%$ survival, respectively. Those with acute HEV (50\%), DILI (37.5\%) and flare of Wilson disease $(37.5 \%)$ have low survival. Half of the pediatric ACLF have one or more organ failure, with the commonest being cerebral and renal. Presence of $\geq 3$ organ failures was associated with poor outcome. Outcome was poor in those with AKI and grade 3-4 HE. The presence of AKI increases the likelihood of death several folds [204]. Among the prognostic models evaluated in ACLF, APACHE-III, SOFA and CLIF SOFA score have been described in children [196, 197]. AARC score was recently shown to have excellent prognostic value in adult ACLF cohort [3]. Serum creatinine ( $\mathrm{SCr}$ ) value included in AARC score is unreliable as children have lower SCr, which is further accentuated due to malnutrition, sarcopenia and decreased endogenous production. A pediatric modification of AARC score was made replacing the absolute $\mathrm{SCr}$ with grades of AKI [204]. Both the AARC score (AUROC 0.945) and its pediatric modification (AUROC 0.951) were superior to the other prognostic scores in pediatric ACLF cohort. A cutoff score of 11 or more identified poor outcome with $87.5 \%$ sensitivity and 90.6\% specificity. Poor outcome group showed a rise, while the good outcome group showed decline in AARC-ACLF score at day 4 ( $\triangle$ AARC-ACLF: Poor outcome: $1 \pm 1.1$ vs. Good outcome: $-0.6 \pm 0.9, p<0.0005)$ [204]. 
The proposal for a definition of ACLF in children by the APASL is the first such attempt to address the issue of ACLF in children. Hope this will be enriched by further large and multicentric studies in future.

\section{Recommendations}

\subsection{Acute-on-chronic liver failure in children.}

8.1. Pediatric ACLF is not uncommon $(1, \mathrm{~B})$.

8.2. The most common underlying liver diseases presenting as ACLF in children are Wilson disease and autoimmune liver disease $(1, \mathrm{~B})$.

8.3. The most common acute precipitating events are viral (HAV, HEV, HBV) hepatitis and flare of underlying disease/hepatotoxic drugs $(1, \mathrm{~B})$.

8.4. Standardized definitions of disease flare, as a precipitating event need to be further updated $(2, \mathrm{C})$.

8.5. The existing definition of ACLF can be used to diagnose ACLF in children. However, there is an urgent need to generate more multicentric data from prospective studies.

8.6. Clinical diagnosis of hepatic encephalopathy, though difficult, is important for diagnosis/prognosis of pediatric ACLF. For diagnosis of HE in older children, West Haven scale can be used. For children less than 3 years, modified $\mathrm{HE}$ assessment scale can be used $(2, \mathrm{C})$.

8.7. Clinical and/or radiological ascites can be used for defining ACLF in children (2, B).

8.8. Short-term (28-day) outcome is poor in approximately $33 \%$ of pediatric ACLF subjects $(2, \mathrm{~B})$.

8.9. One or more extrahepatic organ failures are seen in around half of children with $\operatorname{ACLF}(1, \mathrm{~B})$.

8.10. Acute kidney injury and grade $3-4 \mathrm{HE}$ are most common extrahepatic organ failures in pediatric ACLF.

8.11. Half the cases of ACLF at presentation have AKI. The presence of AKI increases the risk of poor outcome by several folds $(2, \mathrm{~B})$.

8.12. AARC-ACLF model is an easy, bedside, dynamic prognostic model for pediatric $\operatorname{ACLF}(B, 2)$.

8.13. A score more than or equal to 11 needs urgent listing and evaluation for liver transplant $(2, \mathrm{~B})$.

8.14. Pediatric modification of these scores may be useful $(2, \mathrm{C})$.

\section{Conclusions}

In summary, the field of ACLF has moved very rapidly in the past 5 years. The availability of large volume of published data from the East and the West has allowed reassessing the initial definitions. The need for having a well-defined homogenous population of patients, with wellcharacterized acute and chronic insult and which would reflect the term acute-on-chronic liver failure, is at the core. Attempts to abrogate, ameliorate or reverse the ongoing injury would allow return of hepatic synthetic functions and reversal of the liver damage. Early predictors of mortality and non-reversibility of the condition would pave way to offer priority liver transplantation to such patients. An attempt to converge the thoughts from the East and West is possibly the only way forward to achieve more scientific and timely interventions for such seriously sick patients.

Acknowledgements The authors would like to thank Prof Rakesh Aggarwal, Irene Paulson, Ankit Bharadwaj, Shakti Choudhury, Vinay Kumar, Karan Kumar, Harshvardhan Thevetia, Apurva Pandey, Manjul Mishra, Rakesh Kumar Jagdish and R K Gulati for their continued support in developing the consensus data as well as manuscript.

\section{Compliance with ethical standards}

Conflict of interest Shiv Kumar Sarin et al. declare that they have no conflict of interest.

Ethical approval All procedures followed were in accordance with the ethical standards of the responsible committee on human experimentation (institutional and national) and with the Declaration of Helsinki 1975, as revised in 2008. The AARC registry for ACLF was approved by the Institutional Ethical Review board at the nodal centre i.e. ILBS New Delhi and all the participating centres also had neccessary approval from respective ethical board.

Informed consent Informed consent was obtained from all 3356 patients for being included in the study.

Open Access This article is distributed under the terms of the Creative Commons Attribution 4.0 International License (http://creativeco mmons.org/licenses/by/4.0/), which permits unrestricted use, distribution, and reproduction in any medium, provided you give appropriate credit to the original author(s) and the source, provide a link to the Creative Commons license, and indicate if changes were made.

\section{References}

1. Sarin SK, Chandan K, Zaigham A, et al. Acute-on-chronic liver failure: consensus recommendations of the Asian Pacific Association for the study of the liver (APASL). Hepatol Int 2014;8:453-471

2. Sarin SK, Kumar A, et al. Acute-on-chronic liver failure: consensus recommendations of the Asian Pacific Association for the study of the liver (APASL). Hepatol Int 2009;3(269):282

3. Choudhury A, A Jindal SK, Sarin, for the APASL ACLF Working Party, et al. Liver failure determines the outcome in patients of Acute-on-Chronic Liver Failure (ACLF)-comparison of APASL-ACLF Research Consortium (AARC) and CLIF-SOFA model. Hepatol Int 2017;11(5):461-71

4. Atkins D, Best D, Briss PA, et al. Grading quality of evidence and strength of recommendations. BMJ 2004;328:1490

5. O'Grady JG, Schalm SW, Williams R. Acute liver failure: redefining the syndromes. Lancet 1993;342:273-275

6. Jalan R, Pavesi M, Wendon J, CANONIC Study Investigators; EASLCLIF Consortium, et al. The CLIF Consortium Acute Decompensation score (CLIF-C ADs) for prognosis of hospitalised cirrhotic patients without acute-on-chronic liver failure. J Hepatol 2015;62(4):831-840

7. Sen S, William R, Jalan R. The pathophysiological basis of acuteon- chronic liver failure. Liver 2002;22(Suppl 2):5-13 
8. Wlodzimirow KA, Eslami S, Abu-Hanna A, et al. A systematic review on prognostic indicators of acute on chronic liver failure and their predictive value for mortality. Liver Int 2013;33(1):40-52

9. Bajaj JS, Moreau R, Kamath PS, Vargas HE, Arroyo V, Reddy KR, Szabo G, Tandon P, Olson J, Karvellas C, Gustot T, Lai JC, Wong F. Acute-on-chronic liver failure: getting ready for prime time? Hepatology 2018;68(4):1621-1632 PMID:29689120

10. Ferenci P, Lockwood A, Mullen K, et al. Hepatic encephalopathy- definition, nomenclature, diagnosis and quantification: final report of the working party at the 11th World Congress of Gastroenterology, Vienna 1998. Hepatology 2002;25(3):716-721

11. Moreau R, Jalan R, Gines P, et al. Acute-on-chronic liver failure is a distinct syndrome that develops in patients with acute decompensation of cirrhosis. Gastroenterology 2013;144:1426-1437

12. Xu L, Tu Z, Xu G, et al. Epirubicin directly promotes hepatitis $\mathrm{B}$ virus (HBV) replication in stable $\mathrm{HBV}$-expressing cell lines: a novel mechanism of HBV reactivation following anticancer chemotherapy. Mol Med Rep 2014;9(4):1345-1350

13. Mikulska M, Nicolini L, Signori A, et al. Hepatitis B reactivation in $\mathrm{HBsAg}$ negative/HBcAb positive allogeneic hematopoietic stem cell transplant recipients: risk factors and outcome. Clin Microbiol Infect 2014;15:8. https://doi. org/10.1111/1469-0691.12611

14. Pei R, Grund S, Verheyen J, et al. Spontaneous reactivation of hepatitis B virus replication in a HIV coinfected patient with isolated anti-hepatitis B core antibodies. Virol J 2014;11:9. https://doi.org/10.1186/1743-422X-11-9

15. Magalhaes RK, Ferreira D, Salgado M, et al. Hepatitis B virus reactivation after chemotherapy in an HIV positive patient. J Clin Gastroenterol 2013;47(6):565-566

16. Lim SG, Wai CT, Rajnakova A, Kajiji T, Guan R. Fatal hepatitis $B$ reactivation following discontinuation of nucleoside analogues for chronic hepatitis B. Gut 2002;51(4):597-599

17. Martin ST, Cardwell SM, Nailor MD, Gabardi S. Hepatitis B reactivation and Rituximab: A new boxed warning and considerations for solid organ transplantation. Am J Transpl 2014. https://doi.org/10.1111/ajt.12649

18. Kusumoto S, Tanaka Y, Mizokami M, Ueda R. Strategy for preventing hepatitis $\mathrm{B}$ reactivation in patients with resolved HBV infection following rituximab containing chemotherapy. Hepatology 2013. https://doi.org/10.1002/hep.26963

19. Tsutsumi Y, Yamamoto Y, Shimono J, Ohhigashi H, Teshima T. Hepatitis B virus reactivation with rituximab containing regimen. World J Hepatol 2013;5(11):612-662

20. Huang M, Huang L. Reactivation of hepatitis $C$ viral infection after treatment with infliximab. J Clin Gastroenterol 2014;48(2):189-190

21. Sagnelli E, Pisaturo M, Stanzione M, Messina V, Alessio L, Sagnelli C, et al. Clinical presentation, outcome and response to therapy among patients with acute exacerbation of chronic hepatitis C. Clin Gastroenterol Hepatol 2013;11(9):1174-1180

22. Radha Krishna Y, Saraswat VA, Das K, Himanshu G, Yachha SK, Aggarwal R, Choudhari G. Clinical features and predictors of outcome in acute hepatitis A and hepatitis $\mathrm{E}$ virus hepatitis on cirrhosis. Liver Int 2009;29(3):392-398

23. Mahtab MA, Rahman S, Khan M, Karim MF. Hepatitis E virus is a leading cause of acute-on-chronic liver disease: experience from a tertiary center in Bangladesh. Hepatobiliary Pancreat Dis Int 2009;8(1):50-52

24. Kumar M, Sharma BC, Sarin SK. Hepatitis E virus as an etiology of acute exacerbation of previously unrecognized asymptomatic patients with hepatitis B virus related chronic liver disease. J Gastroenterol Hepatol 2008;23(6):83-87

25. Acharya SK, Sharma PK, Singh R, Mohanty SK, Madan K, Jha JK, et al. Hepatitis E virus (HEV) infection in patients with cirrhosis is associated with rapid decompensation and death. J Hepatol 2007;46:387-394

26. Kim AY, Chung RT. Bacterial, Parasitic and Fungal infections of the liver, including liver abscess. In: Feldman M, Friedman L, Brandt LJ, editors. Sleisenger and Fordtran's Gastrointestinal and Liver Disease. 9th ed. Philadelphia: WB Saunders; 2010. 1351

27. Moreau R, Arroyo V. Acute-on-chronic liver failure: a new clinical entity. Clin Gastroenterol Hepatol 2014. https://doi. org/10.1016/j.cgh.2014.02.027.

28. Garg H, Kumar A, Garg V, Sharma P, Sharma BC, Sarin SK. Clinical profile and predictors of mortality in patients of acuteon-chronic liver failure. Dig Liver Dis 2012;44(2):166-171

29. Wasmuth HE, Kunz D, Yagmur E, Timmer-Stranghöner A, Vidacek D, Siewert E, et al. Patients with acute-on-chronic liver failure display "sepsis- like" immune paralysis. J Hepatol 2005;42(2):195-201

30. Duseja A, Chawla YK, Dhiman RK, Kumar A, Choudhary N, Taneja S. Non-Hepatic insults are common precipitants in patients with acute-on-chronic liver failure. Dig Dis Sci 2010;55(11):3188-3192

31. Lo Re V III, Carbonari DM, Lewis JD, et al. Oral azole antifungal medications and risk of acute liver injury, overall and by chronic liver disease status. Am J Med 2016;129:283-291

32. Chalasani N, Bonkovsky HL, Fontana R, et al. Features and outcomes of 899 patients with drug-induced liver injury: the DILIN Prospective Study. Gastroenterology 2015;148:1340-1352

33. Watkins PB, Seligman PJ, Pears JS, et al. Using controlled clinical trials to learn more about acute drug-induced liver injury. Hepatology 2008;48:1680-1689

34. Lewis JH, Stine JG. Review article: prescribing medications in patients with cirrhosis - a practical guide. Aliment Pharmacol Ther 2013;37:1132-1156

35. Tarantino G, Conca P, Basile V, et al. A prospective study of acute drug-induced liver injury in patients suffering from nonalcoholic fatty liver disease. Hepatol Res 2007;37:410-415

36. Pais R, Rusu E, Ratziu V. The impact of obesity and metabolic syndrome on chronic hepatitis B and drug-induced liver disease. Clin Liver Dis 2014;18:165-178

37. Hoffmann CJ, Charalambous S, Thio CL, et al. Hepatotoxicity in an African antiretroviral therapy cohort: the effect of tuberculosis and hepatitis B. Aids 2007;21:1301-1308

38. Thulstrup AM, Molle I, Svendsen N, et al. Incidence and prognosis of tuberculosis in patients with cirrhosis of the liver. A Danish nationwide population based study. Epidemiol Infect 2000;124:221-225

39. Lin YT, Wu PH, Lin CY, et al. Cirrhosis as a risk factor for tuberculosis infection-a nationwide longitudinal study in Taiwan. Am J Epidemiol 2014;180:103-110

40. Wang N-T, Huang Y-S, Lin M-H, Huang B, Perng C-L, Lin $\mathrm{H}-\mathrm{C}$. Chronic hepatitis B infection and risk of antituberculosis drug-induced liver injury: systematic review and meta-analysis. J Chin Med Assoc 2016;79:368-374

41. Kim WS, Lee SS, Lee CM, Kim HJ, Ha CY, Kim HJ, et al. Hepatitis $\mathrm{C}$ and not Hepatitis $\mathrm{B}$ virus is a risk factor for anti-tuberculosis drug induced liver injury. BMC Infect Dis 2016;16:50

42. Qin G, Shao JG, Zhu YC, et al. Population-representative incidence of acute-on-chronic liver failure. A prospective crosssectional study. J Clin Gastroenterol 2016;50:670-675

43. Shi Y, Yang Y, Hu Y, et al. Acute-on-chronic liver failure precipitated by hepatic injury is distinct from that precipitated by extrahepatic insults. Hepatology 2015;62:232-242

44. Shalimar, Saraswat V, Singh SP, et al. Acute-on-chronic liver failure in India: the Indian National Association for Study of 
the Liver consortium experience. J Gastroenterol Hepatol 2016;31:1742-1749

45. Yeoman AD, O'Grady JG, Heneghan MA. Prognosis of acute severe autoimmune hepatitis (AS-AIH): the role of corticosteroids in modifying outcome. J Hepatol 2014;61:876-882

46. Stravitz RT, Lefkowitch JH, Fontana RJ, Gershwin ME, Leung PS, Sterling RK, et al. Autoimmune acute liver failure: proposed clinical and histological criteria. Hepatology 2011;53:517-526

47. Anand L, Choudhury A, Bihari C, Sarin SK, for APASL ACLF (AARC) working party. Flare of Autoimmune Hepatitis causing acute on chronic liver failure (ACLF): diagnosis and response to corticosteroid therapy. Hepatology 2018. https://doi.org/10.1002/ hep.30205 (Epub ahead of print, PMID: 30113706 )

48. Ripoll C, Groszmann R, Garcia-Tsao G, Grace N, Burroughs A, Planas R, et al. Hepatic pressure gradient predicts clinical decompensation in patients with compensated cirrhosis. Gastroenterol 2007;133:481-488

49. Amitrano L, Guardascione MA, Martino R, Manguso F, Menchise A, Balzano A. Hypoxic hepatitis occurring in cirrhosis after variceal bleeding: still a lethal disease. J Clin Gastroenterol 2012;46(7):608-612

50. Bruns T, Zimmermann HW, Stallmach A. Risk factors and outcome of bacterial infections in cirrhosis. World J Gastroenterol 2014;20(10):2542-2554

51. Kim RD, Kim JS, Watanabe G, Mohuczy D, Behrns KE. Liver regeneration and the atrophy-hypertrophy complex. Semin Interv Radiol 2008;25:92-103

52. Langlet P, Escolano S, Valla D, Coste-Zeitoun D, Denie C, Mallet $\mathrm{A}$, et al. Clinicopathological forms and prognostic index in Budd-Chiari syndrome. J Hepatol 2003;39:496-501

53. Desmet VJ, Roskams T. Cirrhosis reversal: a duel between dogma and myth. J Hepatol 2004;40:860-867

54. Wanless IR, Wong F, Blendis LM, Greig P, Heathcote EJ, Levy G. Hepatic and portal vein thrombosis in cirrhosis: possible role in development of parenchymal extinction and portal hypertension. Hepatology 1995;21(5):1238-1247

55. Kim MY, Cho MY, Baik SK, Park HJ, Jeon HK, Im CK, et al. Histological subclassification of cirrhosis using the Laennac fibrosis scoring system correlates with clinical stage and grade of portal hypertension. J Hepatol 2011;55(5):1004-1009

56. Rastogi A, Maiwall R, Bihari C, Ahuja A, Kumar A, Singh T, et al. Cirrhosis etiology and laennac staging system correlate with high portal pressure. Histopathology 2013;62(5):731-741

57. Libbrecht L, Desmet V, Van Damme B, Roskams T. Deep intralobular extension of human hepatic "progenitor cells" correlates with parenchymal inflammation in chronic viral hepatitis: can "progenitor cells" migrate? J Pathol 2000;192:373-378

58. de Graaf EL, Kench J, Dilworth P, Shackel NA, Strasser S, Joseph D, et al. Grade of deceased donor liver macrovesicular steatosis impacts graft and recipient outcomes more than the donor risk index. J Gastroenterol Hepatol 2012;27(3):540-546

59. Gabrielli M, Moisan F, Vidal M, Duarte I, Jiménez M, Izquierdo $\mathrm{G}$, et al. Steatotic livers. Can we use them in OLTX? Outcome data from a prospective baseline liver biopsy study. Ann Hepatol 2012;11(6):891-898

60. Angulo P, Keach JC, Batts KP, Lindor KD. Independent predictors of liver fibrosis in patients with nonalcoholic steatohepatitis. Hepatology 1999;30(6):1356-1362

61. Loomba R, Sanyal A. The global NAFLD epidemic. Nat Rev Gastroenterol Hepatol 2013;10(11):686-690

62. Laleman W, Verbeke L, Meersseman P, Wauters J, van Pelt J, Cassiman D, et al. Acute-on-chronic liver failure: current concepts on definition, pathogenesis, clinical manifestations and potential therapeutic interventions. Expert Rev Gastroenterol Hepatol 2011;5(4):523-537
63. Rastogi A, Kumar A, Sakhuja P, Bihari C, Gondal R, Hissar $\mathrm{S}$, et al. Liver histology as predictor of outcome in patients with acute-on-chronic liver failure (ACLF). Virchows Arch 2011:459(2):121-127

64. Kalambokis G, Manousou P, Vibhakorn S, Marelli L, Cholongitas E, Senzolo M, et al. Transjugular liver biopsy-indications, adequacy, quality of specimens, and complications - a systematic review. J Hep 2007;47:284-294

65. Myers RP, Elkashab M, Ma M, et al. Transient Elastography for noninvasive assessment of liver fibrosis: a multicenter Canadian study. Can J Gastroenterol 2010;24(11):661-670

66. Kedarisetty CK, Bhardwaj A, Garg H, Wagle P, Sarin SK. Reduction in liver stiffness at one month is a predictor of survival in acute-on-chronic liver failure. In: Presented as poster at APASL annual conference 2014, Brisbane

67. Jha AK, Nijhawan S, Rai RR, Nepalia S, Jain P, Suchismita A. Etiology, clinical profile and inhospital mortality of acute-onchronic liver failure: a prospective study. Indian J Gastroenterol 2013;32(2):108-114

68. Seto WK, Lai CL, Yuen MF. Acute-on-chronic liver failure in chronic hepatitis B. J Gastroenterol Hepatol 2012;27(4):662-669

69. Adams LA, Lymp JF, Sanderson SO, Lindor KD, Feldstein A, Angulo $P$. The natural history of nonalcoholic fatty liver disease: a population based cohort study. Gastroenterology 2005;129:113-121

70. Caldwell SH, Crespo DM. The spectrum expanded: cryptogenic cirrhosis and natural history of nonalcoholic fatty liver disease. J Hepatol 2004;40:578-584

71. Bhala N, Angulo P, Van der Poorten D, Lee E, Hui JM, Saracco G, Adams LA, et al. The Natural history of Nonalcoholic fatty liver disease with advanced fibrosis or cirrhosis: an international collaborative study. Hepatology 2011;54:1208-1216

72. Sheth SG, Flamm SL, Gordon FD, Chopra S. AST/ALT ratio predicts cirrhosis in patients with chronic hepatitis $\mathrm{C}$ virus infection. Am J Gastroenterol 1998;93(1):44-48

73. Kruger FC, Daniels CR, Kidd M, Swart G, Brundyn K, van Rensberg C, Kotze M. APRI: a simple bedside marker of advanced fibrosis that can avoid liver biopsy in patients with NAFLD/ NASH. S Afr Med J 2011;101(7):477-480

74. Berzigotti A, Garcia-Tsao G, Bosch J, Grace ND, Burroughs AK, Morillas R, Portal Hypertension Collaborative Group, et al. Obesity is an independent risk factor for clinical decompensation in patients with cirrhosis. Hepatology. 2011;54:555-561

75. Duseja A, De A, Taneja S, Choudhury A, Devarbhavi H, Hu J, et al. Prevalence of metabolic risk factors and its impact on the severity and outcome of patients with alcohol related acute-onchronic liver failure (ACLF- A case control study from the AARC data base. AASLD abstract. Hepatol 2018;S1

76. Choudhury AK, Mahtab AM, Sarin SK, for ACLF Working Party. Acute on Chronic Liver Failure (ACLF) has a better long-term survival than Acute decompensation- a study of 4897 patients from APASL ACLF Research Consortium (AARC) with a follow-up of 5 years. AASLD abstract. Hepatol 2017;S1

77. Choudhury A, Kumar M, Sarin SK, APASL ACLF working party. Systemic inflammatory response syndrome in acute on chronic liver failure- relevance of 'Golden Window' - a prospective study. J Gastroenterol Hepatol 2017;32(12):1989-1997. https ://doi.org/10.1111/jgh.13799 (PMID: 28374414)

78. Katoonizadeh A, Laleman W, Verslype C, Wilmer A, Maleux G, Roskams T, Nevens F. Early features of acute-on-chronic alcoholic liver failure: a prospective cohort study. Gut 2010;59(11):1561-1569

79. Jalan R, Mookerjee RP. Acute-on-chronic liver failure: an early liver biopsy is essential? Gut 2010;59(11):1455-1456

80. Hoffman A, Rahman F, Prengel S, Schuchmann M, Gotz M, Moehler M, Galle PR, Li Z, Kalloo AN, Kiesslich R. Mini-laparoscopy in the endoscopy unit: safety and outcomes in over one 
thousand patients. World J Gastrointest Endosc 2011;3(1):6-10 PMID: 21258600

81. Katoonizadeh A, Laleman W, Verslype C, Wilmer A, Maleux G, Roskams T, Nevens F. Early features of acute-on-chronic alcoholic liver failure: a prospective cohort study. Gut 2010;59(11):1561-1569

82. Rastogi A, Maiwall R, Bihari C, Trehanpati N, Pamecha V, Sarin SK. Two-tier regenerative response in liver failure in humans. Virchows Arch 2014;464(5):565-573

83. Rastogi A, Bihari C, Maiwall R, Ahuja A, Sharma MK, Kumar A, Sarin SK. Hepatic stellate cells are involved in the pathogenesis of acute-on-chronic liver failure. Virchows Arch 2012;461(4):393-398

84. Garg V, Garg H, Khan A, Trehanpati N, Kumar A, Sharma BC, et al. Granulocyte colony stimulating factor mobilizes CD $34+$ cells and improves survival of patients with acute-onchronic liver failure. Gastroenterology 2012;142(3):505-512

85. Saxena P, Bihari C, Rastogi A, Agarwal S, Anand L, Sarin SK. Sonoclot signature analysis in patients with liver disease with conventional coagulation studies. Adv Hematol 2013;2013:23751

86. New York Heart Association Inc. New York Heart Association Classification of Heart Failure. Diseases of the Heart and Blood Vessels: Nomenclature and Criteria for Diagnosis. 6th ed. Boston: Little Brown; 1964. 114

87. Jeppesen JB, Mortensen C, Bendtsen F, Møller S. Lactate metabolism in chronic liver disease. Scand J Clin Lab Invest 2013;73(4):293-299

88. Cardoso NM, Silva T, Basile-Filho A, Mente ED, Castro-e-Silva O. A new formula as a predictive score of post-liver transplantation outcome: postoperative MELD-lactate. Transpl Proc 2014;46(5):1407-1412

89. Cordoba J, Ventura-Cots M, Simón-Talero M, Amorós A, Pavesi M, Vilstrup H, Angeli P, Domenicali M, Ginés P, Bernardi M, Arroyo V, CANONIC Study Investigators of EASL-CLIF Consortium. Char-acteristics, risk factors, and mortality of cirrhotic patients hospital-ized for hepatic encephalopathy with and without acute-on-chronic liver failure (ACLF). J Hepatol 2014;60(2):275-281

90. Levy MM, Fink MP, Marshall JC, Abraham E, Angus D, Cook D, et al. $2001 \mathrm{SCCM} / \mathrm{ESICM} / \mathrm{ACCP} / \mathrm{ATS} / \mathrm{SIS}$ international sepsis definitions conference. Crit Care Med 2003;31:1250-1256

91. Gustot T, Felleiter P, Pickkers P, Sakr Y, Rello J, Velissaris D, et al. Impact of infection on the prognosis of critically ill cirrhotic patients: results from a large worldwide study. Liver Int 2014;7:15. https://doi.org/10.1111/liv.12520.

92. Arvaniti V, D'Amico G, Fede G, Manousou P, Tsochatzis E, Plequezuelo M, Burroughs AK. Infections in patients with cirrhosis increase mortality four- fold and should be used in determining prognosis. Gastroenterology 2010;139:1246-1256

93. Hoque R, Farooq A, Mehal WZ. Sterile inflammation in the liver and pancreas. J Gastroenterol Hepatol 2013;28:61-67

94. Chovatiya R, Medzhitov R. Stress, inflammation, and defense of homeostasis. Mol Cell 2014;54:281-288

95. Thabut D, Massard J, Moreau R, et al. Model for end-stage liver disease score and systemic inflammatory response are major prognostic factors in patients with cirrhosis and acute functional renal failure. Hepatology 2007;46:1872-1882

96. Jalan R, Stadlbauer V, Sen S, Cheshire L, Chang YM, Mookerjee RP. Role of predisposition, injury, response and organ failure in the prognosis of patients with acute-on-chronic liver failure: a prospective cohort study. Crit Care 2012;16(6):R227

97. Granja C, Póvoa P, Lobo C, Teixeira-Pinto A, Carneiro A, CostaPereira A. The predisposition, infection, response and organ failure (Piro) sepsis classification system: results of hospital mortality using a novel concept and methodological approach.
PLoS One 2013;8(1):e53885. https://doi.org/10.1371/journ al.pone. 0053885

98. Hotchkiss RS, Monneret G, Payen D. Sepsis induced immunosuppression: from cellular dysfunctions to immunotherapy. Nat Rev Immunol 2013;13(12):862-874

99. Duseja A, Chawla YK, Dhiman RK, Kumar A, Choudhary N, Taneja S. Non-hepatic insults are common precipitants in patients with acute-on-chronic liver failure. Dig Dis Sci 2010;55(11):3188-3192

100. Khanam A, Trehanpati N, Garg V, Kumar C, Garg H, Sharma $\mathrm{BC}$, Sarin SK. Altered frequencies of dendritic cells and IFN$\gamma$ secreting T cells with granulocyte colony stimulating factor (GCSF) therapy in acute-on-chronic liver failure. Liver Int 2014;34(4):505-513

101. Kox WJ, Volk T, Kox SN, Volk HD. Immunomodulatory therapies in sepsis. Intensive Care Med 2000;26(Suppl 1):S124-S128

102. Wasmuth HE, Kunz D, Yagmur E, Timmer- Stranghöner A, Vidacek D, Siewert E, et al. Patients with acute-on-chronic liver failure display "sepsis- like" immune paralysis. J Hepatol 2005;42(2):195-201

103. Jindal A, Sarin SK. Acute Kidney Injury (AKI) at admission and its response to terlipressin as a predictor of mortality in patients with acute-on-chronic liver failure (ACLF). J Hepatol 2013;58(S1):S89

104. Gines P, Schrier RW. Renal failure in cirrhosis. N Engl J Med 2009;361:1279-1290

105. Martín-Llahí M, Guevara M, Torre A, Fagundes C, Restuccia T, Gilabert R, et al. Prognostic importance of the cause of renal failure in patients with cirrhosis. Gastroenterology 2011;140:488-496

106. Stadlbauer V, Wright G, Banaji M, Mukhopadhyay A, Mookerjee $\mathrm{R}$, Moore $\mathrm{K}$, et al. Relationship between activation of the sympathetic nervous system and renal blood flow autoregulation in cirrhosis. Gastroenterology 2008;134:111-119

107. Kumar A, Das K, Sharma P, Mehta V, Sharma BC, Sarin SK. Hemodynamic studies in acute-on-chronic liver failure. Dig Dis Sci 2009;54(4):869-878. https://doi.org/10.1007/s1062 0-008-0421-9.

108. Cárdenas A, Ginès P. Acute-on-chronic liver failure: the kidneys. Curr Opin Crit Care 2011;17(2):184-189

109. Maiwall R, Sarin SK, Kumar S, for APASL ACLF Research Consortium (AARC) working party, et al. Development of predisposition, injury, response, organ failure model for predicting acute kidney injury in acute on chronic liver failure. Liver Int 2017. https://doi.org/10.1111/liv.13443 (Epub ahead of print, PMID: 28393476)

110. Maiwall R, Kumar S, Vashishtha C, Kumar M, Garg H, Nayak $\mathrm{SL}$, et al. Acute kidney injury (AKI) in patients with Acuteon-chronic Liver failure (ACLF) is different from patients with cirrhosis. Hepatology 2013;58(4 Suppl):36A-91A

111. Wan ZH, Wang JJ, You SL, Liu HL, Zhu B, Zang H, et al. Cystatin $\mathrm{C}$ is a biomarker for predicting acute kidney injury in patients with acute-on-chronic liver failure. World J Gastroenterol 2013;19(48):9432-9438

112. Xing T, Zhong L, Chen D, Peng Z. Experience of combined liver-kidney transplantation for acute-on-chronic liver failure with renal dysfunction. Transpl Proc 2013;45(6):2307-2313

113. Maiwall R, Chandel SS, Wani Z, Kumar S, Sarin SK. SIRS at admission is a predictor of AKI development and mortality in hospitalized patients with severe alcoholic hepatitis. Dig Dis Sci 2016;61(3):920-929

114. Arora V, Maiwall R, Choudhury A, Jain P, Kumar G, Sarin SK. Terlipressin is superior to Noradrenaline in the management of acute kidney injury (AKI) in patients with ACLF. Terlipressin is superior to Noradrenaline in the management of acute kidney 
injury (AKI) in patients with ACLF. J Hepatol 2017;66(1):S563 (in press)

115. Gonwa TA, Wadei HM. The challenges of providing renal replacement therapy in decompensated liver cirrhosis. Blood Purif 2012;33:144-148

116. Cardoso FS, Gottfried M, Tujios S, Olson JC, Karvellas CJ, US Acute Liver Failure Study Group. Continuous renal replacement therapy is associated with reduced serum ammonia levels and mortality in acute liver failure. Hepatology 2017;31:15. https:// doi.org/10.1002/hep.29488 (Epub ahead of print)

117. Donovan JP, Schafer DF, Shaw BW Jr, Sorrell MF. Cerebral edema and increased intracranial pressure in chronic liver disease. Lancet 1998;351:719-721

118. Gupta T, Dhiman RK, Ahuja CK, Agrawal S, Chopra M, Kalra N, Duseja A, Taneja S, Khandelwal N, Chawla Y. Characterization of cerebral edema in acute-on chronic liver failure. J Clin Exp Hepatol 2017;7:190-197.

119. Butterworth RF, McPhail MJW. L-ornithine L-aspartate (LOLA) for hepatic encephalopathy in cirrhosis: results of randomized controlled trials and meta-analyses. Drugs 2019;79(Suppl 1):31-37

120. Shawcross DL, Sharifi Y, Canavan JB, Yeoman AD, Abeles RD, Taylor NJ, et al. Infection and systemic inflammation, not ammonia, are associated with grade 3/4 hepatic encephalopathy, but not mortality in cirrhosis. J Hepatol 2011;54:640-649

121. Guevara M, Baccaro ME, Torre A. Hyponatremia is a risk factor of hepatic encephalopathy in patients with cirrhosis: a prospective study with time-dependent analysis. Am J Gastroenterol 2009;104:1382-1389

122. Verma A, Choudhury AK, Sarin SK, for APASL ACLF Working party, et al. Hepatic encephalopathy and ammonia can predict 28 day mortality in acute on chronic liver failure in a dynamic manner-results of multination study from APASAL-ACLF Research Consortium. AASLD abstract. Hepatol 2017;S1

123. Tripodi A, Primignani M, Chantarangkul V, Dell'Era A, Clerici M, de Franchis R, et al. An imbalance of pro- vs anti-coagulation factors in plasma from patients with cirrhosis. Gastroenterology 2009; $137: 2105-2111$

124. Caldwell SH, Hoffman M, Lisman T, Macik BG, Northup PG, Reddy KR, et al. Coagulation disorders and hemostasis in liver disease: pathophysiology and critical assessment of current management. Hepatology 2006;44:1039-1046

125. Blasi A, Calvo A, Prado V, Reverter E, Carlos Reverter J. Hernández-Tejero M Coagulation failure in patients with Acuteon-Chronic Liver Failure (ACLF) and decompensated cirrhosis: beyond INR. Hepatology 2018. https://doi.org/10.1002/ hep. 30103

126. Fisher C, Patel VC, Stoy SH, Singanayagam A, Adelmeijer J, Wendon J, et al. Balanced haemostasis with both hypo- and hyper-coagulable features in critically ill patients with acute-onchronic-liver failure. J Crit Care 2018;43:54-60

127. Montalto P, Vlachogiannakos J, Cox DJ, Pastacaldi S, Patch $\mathrm{D}$, Burroughs AK. Bacterial infection in cirrhosis impairs coagulation by a heparin effect: a prospective study. J Hepatol 2002;37:463-470

128. Noris M, Remuzzi G. Uremic bleeding: closing the circle after 30 years of controversies? Blood 1999;94:2569-2574

129. Premkumar M, Saxena P, Mirza R, Baweja S, Sharma CB, Choudhury AK, Kedarisetty CK, Sarin SK. Assessment and relevance of coagulation disorders in patients with acute-on-chronic liver failure during Systemic Inflammatory Response (SIRS) and development of sepsis. Liv Int.2018. https://doi.org/10.1111/ liv.14034 (accepted)

130. Blasi A, Calvo A, Prado V, Reverter E, Carlos Reverter J, Hernández-Tejero M, Aziz F, Amoros A, Cardenas A, Fernández J. Coagulation failure in patients with Acute-on-Chronic Liver
Failure (ACLF) and decompensated cirrhosis: beyond INR. Hepatology 2018. https://doi.org/10.1002/hep.30103

131. Premkumar M, Saxena P, Mirza R, Jain P, Bhatia P, Kumar G, Sukriti S, Bihari C, Choudhury A, Sarin SK. Heparin like effect increases risk of mortality and bleeding tendency in patients with severe alcoholic hepatitis during systemic Inflammatory Response (SIRS) and sepsis. AASLD abstract. Hepatol 2017;S1

132. Wells PS, Anderson DR, Rodger M, Forgie M, Kearon C, Dreyer J, Kovacs G, Mitchell M, Lewandowski B, Kovacs MJ. Evaluation of D-dimer in the diagnosis of suspected deep-vein thrombosis. N Engl J Med 2003;349(13):1227-1235 PubMed PMID: 14507948

133. Garg H, Kumar A, Garg V, Kumar M, Kumar R, Sharma BC, et al. Hepatic and systemic hemodynamic derangements predict early mortality and recovery in patients with acute-on-chronic liver failure. J Gastroenterol Hepatol 2013;28(8):1361-137

134. Kumar V, Choudhury AK, Maiwall R, Mahtab MA, Rahman S, Alam MS, et al. Degree of hemodynamic derangements correlate with poor outcomes in acute on chronic liver failure (ACLF) patients. AASLD abstract. Hepatol 2018;S1

135. Choudhury AK, Sharma MK, Maiwal R, Jain P, Mahtab MA, Chawla YK, et al. Portal hemodynamics predicts the outcome in severe alcoholic hepatitis presenting as acute-on-chronic liver failure. J Hepatol 2016;S1:64-306

136. Kainth S, Kumar M, Choudhury AK, Anand L, Jindal A, Kumar $\mathrm{G}$, et al. Efficacy and safety of carvedilol in patients of acuteon-chronic liver failure with small or no esophageal varices-a placebo control open label randomised trial (NCT02583698). Hepatology 2017;66(Suppl 1):83A

137. Hari A, Nair HK, De Gottardi A, Baumgartner I, Dufour JF, Berzigotti A. Diagnostic hepatic haemodynamic techniques: safety and radiation exposure. Liver Int 2017;37(1):148-154

138. Qi X, Berzigotti A, Cardenas A, Sarin SK. Emerging non-invasive approaches for diagnosis and monitoring of portal hypertension. Lancet Gastroenterol Hepatol 2018;3(10):708-719

139. Qi X, Li Z, Huang J, Zhu Y, Liu H, Zhou F, Liu C, Xiao C, Dong J, Zhao Y, Xu M, Xing S, Xu W, Yang C. Virtual portal pressure gradient from anatomic CT angiography. Gut 2015;64(6):1004-1005

140. Liu F, Ning Z, Liu Y, Liu D, Tian J, Luo H, An W, Huang Y, Zou J, Liu C, Liu C, Wang L, Liu Z, Qi R, Zuo C, Zhang Q, Wang J, Zhao D, Duan Y, Peng B, Qi X, Zhang Y, Yang Y, Hou J, Dong J, Li Z, Ding H, Zhang Y, Qi X. Development and validation of a radiomics signature for clinically significant portal hypertension in cirrhosis (CHESS1701): a prospective multicenter study. E BioMed 2018;36:151-158

141. Arora V, Maiwal R, Thomas SS, RajanV, Ali R, Kumar G, Jain $\mathrm{P}$, Sarin SK. Albumin decreases the incidence of paracentesis induced circulatory dysfunction with less than 5 litres of ascitic tap in acute on chronic liver failure (ACLF) patients: randomized controlled trial (NCT02467348). J Hepatol 2018;68(1):S42-S43

142. Cabrera J, Falcón L, Gorriz E, et al. Abdominal decompression plays a major role in early postparacentesis haemodynamic changes in cirrhotic patients with tense ascites Gut 2001;48:384-389

143. Singh V, Singh A, Singh B, Vijayvergiya R, Sharma N, Ghai A, Bhalla A. Midodrine and clonidine in patients with cirrhosis and refractory or recurrent ascites: a randomized pilot study. Am J Gastroenterol 2013;108(4):560-567

144. Singh V, Kumar B, Nain CK, Singh B, Sharma N, Bhalla A, Sharma AK. Noradrenaline and albumin in paracentesis-induced circulatory dysfunction in cirrhosis: a randomized pilot study. J Intern Med 2006;260(1):62-68

145. Xue R, Duan Z, Liu H, Chen L, Yu H, Ren M, Zhu Y, Jin C, Han $\mathrm{T}$, Gao Z, Meng Q. A novel dynamic model for predicting outcome in patients with hepatitis B virus related acute-on-chronic 
liver failure. Oncotarget 2017;8(65):108970-108980. https://doi. org/10.18632/oncotarget.22447

146. Song DS, Kim TY, Kim DJ, Korean Acute-on-Chronic Liver Failure (KACLiF) Study Group, et al. Validation of prognostic scores to predict short-term mortality in patients with acute-onchronic liver failure. J Gastroenterol Hepatol 2017. https://doi. org/10.1111/jgh.13991 (Epub ahead of print)

147. Chan CA, Fan ST, Lo CM, Liu CL, Chan SC, Ng KK, et al. Liver transplantation for acute-on-chronic liver failure. Hepatol Int 2009;3:571-581

148. Mathurin $\mathrm{P}$, et al. Corticosteroids improve short-term survival in patients with severe alcoholic hepatitis: meta-analysis of individual patient data. Gut 2011;60:255-260

149. Wong RJ, Aguilar M, Gish RG, Cheung R, Ahmed A. The impact of pretransplant hepatic encephalopathy on survival following liver transplantation. Liver Transpl 2015;21:873-880.

150. Yeoman AD, O'Grady JG, Heneghan MA. Prognosis of acute severe autoimmune hepatitis (AS-AIH): the role of corticosteroids in modifying outcome. J Hepatol 2014. https://doi. org/10.1016/j.jhep.2014.05.021.

151. Nakao M, Nakayama N, Uchida Y, Tomiya T, Ido A, Sakaida I, Yokosuka O, Takikawa Y, Inoue K, Genda T, Shimizu M, Terai S, Tsubouchi H, Takikawa H, Mochida S. Nationwide survey for acute liver failure and late-onset hepatic failure in Japan. J Gastroenterol 2017. https://doi.org/10.1007/s00535-017-1394-2 (Epub ahead of print)

152. Yeoman AD, Westbrook RH, Portmann C, O'Grady OJ, Harrison PM, Heneghan MA. Early predictors of corticosteroid treatment failure in icteric presentations of autoimmune hepatitis. Hepatolog. 2011;53:926-934

153. Wang J, Ma K, Han M, Guo W, Huang J, Yang D, Zhao X, Song J, Tian D, Qi J, Huang Y, Ning Q. Nucleoside analogs prevent disease progression in HBV-related acute-on-chronic liver failure: validation of the TPPM model. Hepatol Int 2014;8(1):64-71 (153n)

154. Ma K, Guo W, Han M, et al. Entecavir treatment prevents disease progression in hepatitis B virus-related acute-on-chronic liver failure: establishment of a novel logistical regression model. Hepatol Int 2012;6(4):735-743 (153n)

155. Tao C, Zhongyuan Y, Kumar A, Choudhury for APASL ACLF Research Consortium (AARC). Hepatology 2017; 67 ([ABSTRACT] Supple,ID\#2776063.[155n])

156. Agrawal S, Duseja A, Gupta T, Dhiman RK, Chawla Y. Simple organ failure count versus CANONIC grading system for predicting mortality in acute-on-chronic liver failure. J Gastroenterol Hepatol 2015;30(3):575-581

157. Hoofnagle JH. Reactivation of hepatitis B. Hepatology 2009;49:S156-S165

158. Jindal A, Kumar M, Sarin SK. Management of acute hepatitis B and reactivation of hepatitis B. Liver Int 2013;33(Suppl 1): $164-175$

159. Sun LJ, Yu JW, Zhao YH, Kang P, Li SC. Influential factors of prognosis in lamivudine treatment for patients with acuteon-chronic hepatitis B liver failure. J Gastroenterol Hepatol 2010;25(3):583-590

160. Hwang JP, Lok AS. Management of patients with hepatitis B who require immunosuppressive therapy. Nat Rev Gastroenterol Hepatol 2013. https://doi.org/10.1038/nrgastro.2013.216

161. Kusumoto S, Tanaka Y, Mizokami M, Ueda R. Strategy for preventing hepatitis B reactivation in patients with resolved HBV infection following rituximab containing chemotherapy. Hepatology 2013. https://doi.org/10.1002/hep.26963

162. Jalan R, Gines P, Olson JC, Mookerjee RP, Moreau R, Garcia-Tsao G, et al. Acute-on chronic liver failure. J Hepatol 2012;57(6): 1336-1348
163. Bahirwani R, Shaked O, Bewtra M, Forde K, Reddy KR. Acuteon-chronic liver failure before liver transplantation: impact on post transplant outcomes. Transplantation 2011;92(8):952-957

164. Lin KH, Liu JW, Chen CL, Wang SH, Lin CC, Liu YW, et al. Impacts of pretransplant infections on clinical outcomes of patients with acute-on-chronic liver failure who received living donor liver transplantation. PLoS One 2013;8(9):e72893

165. Pamecha V, Kumar S, Bharathy KG. Liver transplantation in acute-on-chronic liver failure: challenges and an algorithm for patient selection and management. HepatolInt. 2015.

166. Chan AC, Fan ST, Lo CM, Liu CL, Chan SC, Ng KK, et al. Liver transplantation for acute-on-chronic liver failure. Hepatol Int 2009;3(4):571-581

167. Chok KSh, Chan SC, Fung JY, Cheung TT, Chan AC, Fan ST, Lo $\mathrm{CM}$. Survival outcomes of right-lobe living donor transplantation for patients with high model for end stage Liver Disease scores. Hepatobiliary Pancreat Dis Int 2013;12(3):256-262

168. Choudhury AK, Sharma M, Mehtab M, Sarin SK, for APASL ACLF Working party, et al. The decision for liver transplant in acute-on-chronic liver failure (ACLF) - first week is the crucial period-analysis of the APASL ACLF Research Consortium (AARC) prospective data of 1021 patients. J Hepatol 2016;64:S1-S51

169. Gustot T, Fernandez J, Garcia E, for CANONIC Study Investigators of the EASL-CLIF Consortium, et al. Clinical course of acute-on-chronic liver failure syndrome and effects on prognosis. Hepatology 2015;62(1):243-252

170. Wong RJ, Aguilar M, Gish RG, Cheung R, Ahmed A. The impact of pretransplant hepatic encephalopathy on survival following liver transplantation. Liver Transpl 2015;21:873-880

171. Staufer K, Roedl K, Kivaranovic D, Drolz A, Horvatits T, Rasoul-Rockenschaub S, Zauner C, Trauner M, Fuhrmann V. Renal replacement therapy in critically ill liver cirrhotic patients-outcome and clinical implications. Liver Int 2017;37(6):843-850. https://doi.org/10.1111/liv.13389 (Epub 2017 Apr 5).

172. Cheng $\mathrm{CH}$, Lee CF, Soong RS, Wu TH, Chan KM, Chou HS, Wu TJ, Yu MC, Lee WC. Risk factors and clinical outcomes of ventilator-associated pneumonia in patients on the liver transplant waiting list. Transpl Proc. 2012;44(3):762-4.

173. Moon DB, Lee SG, Kang WH, Song GW, Jung DH, Park GC, Cho HD, Jwa EK, Kim WJ, Ha TY, Kim HJ. Adult living donor liver transplantation for acute-on-chronic liver failure in highmodel for end-stage liver disease score patients. Am J Transpl 2017;17(7):1833-1842 (PMID: 28097804-64)

174. Putignano A, Gustot T. New concepts in acute-on-chronic liver failure: implications for liver transplantation. Liver Transpl 2017;23(2):234-243. https://doi.org/10.1002/lt.24654 (Epub 2017 Jan 6. Review. PMID: 27750389-65)

175. Artru F, Louvet A, Ruiz I, et al. Liver transplantation in the most severely ill cirrhotic patients: a multicenter study in acuteon-chronic liver failure grade 3. J Hepatol 2017. https://doi. org/10.1016/j.jhep.2017.06.009.

176. Reddy MS, Rajalingam R, Rela M. Liver transplantation in acuteon-chronic liver failure: lessons learnt from acute liver failure setting. Hepatol Int 2015;9(4):508-513. https://doi.org/10.1007/ s12072-014-9603-z.

177. Sarin SK, Choudhury A. Management of acute-on-chronic liver failure: an algorithmic approach. Hepatol Int 2018;12(5):402416. https://doi.org/10.1007/s12072-018-9887-5.

178. Jalan R, Williams R. Acute-on-chronic liver failure: pathophysiological basis of therapeutic options. Blood Purif 2002;20:252-261

179. Hassanein TI, Tofteng F, Brown RS Jr, McGuire B, Lynch P, Mehta R, et al. Randomized controlled study of extracorporeal 
albumin dialysis for hepatic encephalopathy in advanced cirrhosis. Hepatology 2007;46(6):1853-1862

180. Banares R, Nevens F, Larsen FS, Jalan R, Albillos A, Dollinger $\mathrm{M}$, et al. Extracorporeal albumin dialysis with the molecular adsorbent recirculating system in acute-on-chronic liver failure: the RELIEF trial. Hepatology 2013;57:1153-1162

181. Kribben A, Gerken G, Haag S, Herget-Rosenthal S, Treichel $\mathrm{U}$, Betz C, et al. Effects of fractionated plasma separation and adsorption on survival in patients with acute-on-chronic liver failure. Gastroenterology 2012;142:782-789

182. Mitzner SR, Stange J, Klammt S, Risler T, Erley CM, Bader BD, et al. Improvement of hepatorenal syndrome with extracorporeal albumin dialysis MARS: results of a prospective, randomized, controlled clinical trial. Liver Transpl 2000;6:277-286

183. Catalina MV, Barrio J, Anaya F, Salcedo M, Rincón D, Clemente G, Bañares R. Hepatic and systemic haemodynamic changes after MARS in patients with acute on chronic liver failure. Liver Int 2003;23(Suppl 3):39-43

184. Laleman W, Wilmer A, Evenepoel P, Elst IV, Zeegers M, Zaman $Z$, et al. Effect of the molecular adsorbent recirculating system and Prometheus devices on systemic haemodynamics and vasoactive agents in patients with acute-on-chronic alcoholic liver failure. Crit Care 2006;10:R108

185. Guo LM, Liu JY, Xu DZ, Li BS, Han H, Wang LH, et al. Application of Molecular Adsorbents Recirculating System to remove $\mathrm{NO}$ and cytokines in severe liver failure patients with multiple organ dysfunction syndrome. Liver Int 2003;23(Suppl 3):16-20

186. Atienza Merino G. Evaluation of extracorporeal liver support systems in thetreatment of liver failure: a systematic review. Gastroenterol Hepatol 2010;33(5):352-362

187. Kjaergard LL, Liu J, Als-Nielsen B, Gluud C. Artificial and bioartificial support systems for acute and acute-on-chronic liver failure: a systematic review. JAMA 2003;289(2):217-222

188. Larsen FS, Schmidt LE, Bernsmeier C, et al. High-volume plasma exchange in patients with acute liver failure: an open randomised controlled trial. J Hepatol 2016;64(1):69-78 (PMID: 26325537)

189. Ling Q, Xu X, Wei Q, Liu X, Guo H, Zhuang L, et al. Downgrading MELD improves the outcomes after liver transplantation in patients with acute-on-chronic hepatitis B liver failure. PLoS One 2012;7(1):e30322

190. Xu X, Liu X, Ling Q, Wei Q, Liu Z, Xu X, et al. Artificial liver support system combined with liver transplantation in treatment of patients with acute-on-chronic liver failure. PLoS One 2013;8(3):e58738

191. Duan XZ, Liu FF, Tong JJ, Yang HZ, Chen J, Liu XY, et al. Granulocyte colony stimulating factor therapy improves survival in patients with hepatitis B virus-associated acute-on-chronic liver failure. World J Gastroenterol 2013;19:1104-1110
192. Forbes SJ, Gupta S, Dhawan A. Cell therapy for liver disease: from liver transplantation to cell factory. J Hepatol 2015;62(1S):S157-S169

193. Liao X, AnCheng JY, Zhou QJ, Liao C. Therapeutic effect of autologous bone marrow-derived liver stem cells transplantation in hepatitis B virus-induced liver cirrhosis. Hepatogastroenterology 2013;60(123):406-409

194. Di Campli C, Zocco MA, Saulnier N, et al. Safety and efficacy profile of G-CSF therapy in patients with acute on chronic liver failure. Dig Liver Dis 2007;39:1071-1076

195. Singh V, Sharma AK, Sharma N, Sharma R. Granulocyte colonystimulating factor in severe alcoholic hepatitis: a randomized pilot study. Am J Gastroenterol 2014;109:1417-1423

196. Kedarisetty CK, Anand L, Bhatia V, Sarin SK. Combination of granulocyte colony-stimulating factor and erythropoietin improves outcomes of patients with decompensated cirrhosis. Gastroenterology 2015;148(7):1362.e7-1370.e7

197. Meng F, Francis H, Glaser S, Alpini G. Role of stem cell factor and granulocyte colony-stimulating factor in remodeling during liver regeneration. Hepatology 2012;55(1):209-221

198. Pusic I, Di Persio JF. Update on clinical experience with amd3100, an sdf-1/cxcl12-cxcr4 inhibitor, in mobilization of hematopoietic stem and progenitor cells. Curr Opin Hematol 2010;17:319-326

199. Alam S, Lal BB, Sood V, Rawat D. Pediatric acute-on-chronic liver failure in a specialized liver unit: prevalence, profile, outcome and predictive factors. J Pediatr Gastroenterol Nutr 2016;63(4):400-405. https://doi.org/10.1097/MPG.0000000000 001179

200. Lal J, Thapa BR, Rawal P, Ratho RK, Singh K. Predictors of outcome in acute-on-chronic liver failure in children. Hepatol Int 2011;5:693-697

201. Jagadisan B, Srivastava A, Yachha SK, Poddar U. Acute-onchronic liver disease in children from the developing world: recognition and prognosis. J Pediatr Gastroenterol Nutr 2012;54:77-82

202. Lal BB, Sood V, Khanna R, Alam S. How to identify the need for liver transplantation in pediatric acute-on-chronic liver failure? Hepatol Int. 2018;12(6):552-9.

203. Squires RH Jr, Shneider BL, Bucuvalas J, et al. Acute liver failure in children: the first 348 patients in the pediatric acute liver failure study group. J Pediatr 2006; 148:652-658

204. Lal BB, Alam S, Sood V, Rawat D, Khanna R. Profile, risk factors and outcome of acute kidney injury in pediatric acute-onchronic liver failure. Liver Int 2018;38(10):1777-1784. https:// doi.org/10.1111/liv.13693

Publisher's Note Springer Nature remains neutral with regard to jurisdictional claims in published maps and institutional affiliations.

\section{Affiliations}

Shiv Kumar Sarin ${ }^{1} \cdot$ Ashok Choudhury $^{1} \cdot$ Manoj K. Sharma $^{1} \cdot$ Rakhi Maiwall $^{1} \cdot$ Mamun Al Mahtab $^{2}$. Salimur Rahman ${ }^{2}$ - Sanjiv Saigal ${ }^{3}$ - Neeraj Saraf ${ }^{3}$ - A. S. Soin ${ }^{3}$. Harshad Devarbhavi ${ }^{4}$. Dong Joon Kim $^{5}$. R. K. Dhiman ${ }^{6} \cdot$ Ajay Duseja $^{6} \cdot$ Sunil Taneja $^{6} \cdot$ C. E. Eapen ${ }^{7} \cdot$ Ashish Goel $^{7} \cdot$ Q. Ning ${ }^{8} \cdot$ Tao Chen $^{9} \cdot \mathrm{Ke} \mathrm{Ma}^{8}$. Z. Duan ${ }^{9}$. Chen $\mathrm{Yu}^{9}$. Sombat Treeprasertsuk ${ }^{10}$. S. S. Hamid ${ }^{11}$. Amna S. Butt ${ }^{11}$. Wasim Jafri ${ }^{11}$ - Akash Shukla $^{12}$. Vivek Saraswat ${ }^{13}$. Soek Siam Tan ${ }^{14}$. Ajit Sood ${ }^{15}$. Vandana Midha ${ }^{15}$. Omesh Goyal ${ }^{15} \cdot$ Hasmik Ghazinyan $^{16}$. Anil Arora ${ }^{17}$. Jinhua Hu ${ }^{18}$. Manoj Sahu ${ }^{19}$. P. N. Rao ${ }^{20}$. Guan H. Lee ${ }^{21}$. Seng G. Lim ${ }^{21}$. Laurentius A. Lesmana ${ }^{22}$. Cosmas Rinaldi Lesmana ${ }^{22}$. Samir Shah ${ }^{23}$. V. G. Mohan Prasad ${ }^{24}$. Diana A. Payawal ${ }^{25}$. Zaigham Abbas . $^{26}$. A. Kadir Dokmeci ${ }^{27}$. Jose D. Sollano ${ }^{28}$. Gian Carpio ${ }^{28}$. Ananta Shresta ${ }^{29}$. G. K. Lau ${ }^{30}$. Md. Fazal Karim ${ }^{31}$.

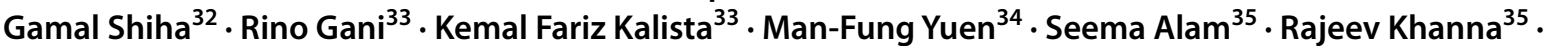


Vikrant Sood ${ }^{35} \cdot$ Bikrant Bihari Lal $^{35} \cdot$ Viniyendra Pamecha $^{36} \cdot$ Ankur Jindal $^{1} \cdot$ V. Rajan ${ }^{1} \cdot$ Vinod Arora $^{1}$. Osamu Yokosuka ${ }^{37}$. Madunil A. Niriella ${ }^{38} \cdot \mathrm{Hai}^{3 i^{39}} \cdot \mathrm{Xiaolong} \mathrm{Qi}^{40}$ - Atsushi Tanaka ${ }^{41}$ - Satoshi Mochida ${ }^{42}$. Dominic Ray Chaudhuri ${ }^{43}$. Ed Gane ${ }^{43} \cdot$ Khin Maung Win $^{44}$. Wei Ting Chen ${ }^{45}$. Mohd. Rela ${ }^{46}$. Dharmesh Kapoor ${ }^{23}$. Amit Rastogi ${ }^{3}$. Pratibha Kale ${ }^{47}$. Archana Rastogi $^{48}$. Chhagan Bihari Sharma ${ }^{48}$. Meenu Bajpai ${ }^{49} \cdot$ Virender Singh $^{6}$. Madhumita Premkumar ${ }^{6}$. Sudhir Maharashi ${ }^{50} \cdot$ A. Olithselvan ${ }^{51}$. Cyriac Abby Philips ${ }^{52}$ - Anshu Srivastava ${ }^{53}$. Surender K. Yachha ${ }^{53}$. Zeeshan Ahmad Wani ${ }^{54}$ - B. R. Thapa ${ }^{55}$. Anoop Saraya ${ }^{56}$. Shalimar ${ }^{56}$. Ashish Kumar ${ }^{17}$. Manav Wadhawan ${ }^{57}$. Subash Gupta ${ }^{58} \cdot$ Kaushal Madan $^{59} \cdot$ Puja Sakhuja ${ }^{60} \cdot$ Vivek Vij61 $^{61}$ Barjesh C. Sharma ${ }^{62}$. Hitendra Garg ${ }^{63}$. Vishal Garg ${ }^{63}$. Chetan Kalal ${ }^{64}$. Lovkesh Anand ${ }^{65}$. Tanmay Vyas ${ }^{66}$. Rajan P. Mathur ${ }^{67}$. Guresh Kumar $^{68}$. Priyanka Jain ${ }^{68}$. Samba Siva Rao Pasupuleti ${ }^{68} \cdot$ Yogesh K. Chawla $^{69} \cdot$ Abhijit Chowdhury $^{70}$. Shahinul Alam ${ }^{2}$. Do Seon Song ${ }^{71}$. Jin Mo Yang ${ }^{71}$. Eileen L. Yoon ${ }^{72}$. APASL ACLF Research Consortium (AARC) for APASL ACLF working Party.

Shiv Kumar Sarin sksarin@ilbs.in

1 Department of Hepatology, Institute of Liver and Biliary Sciences, New Delhi 110070, India

2 Department of Hepatology, Bangabandhu Sheikh Mujib Medical University, Dhaka, Bangladesh

3 Department of Hepatology, Medanta The Medicity, Gurgaon, India

4 Department of Hepatology, St John Medical College, Bangalore, India

5 Department of Internal Medicine, Hallym University College of Medicine, Seoul, South Korea

6 Department of Hepatology, PGIMER, Chandigarh, India

7 Department of Hepatology, CMC, Vellore, India

8 Institute and Department of Infectious Disease, Tongji Hospital, Tongji Medical College, Huazhong University of Science and Technology, Wuhan, China

9 Translational Hepatology Institute Capital Medical University, Beijing You'an Hospital, Beijing, China

10 Department of Medicine, Chulalongkorn University, Bangkok, Thailand

11 Department of Medicine, Aga Khan University Hospital, Karachi, Pakistan

12 Department of Gastroenterology, Lokmanya Tilak Municipal General Hospital and Lokmanya Tilak Municipal Medical College, Sion, Mumbai, India

13 Department of Gastroenterology, SGPGIMS, Lucknow, India

14 Department of Medicine, Hospital Selayang, Bata Caves, Selangor, Malaysia

15 Department of Gastroenterology, DMC, Ludhiana, India

16 Department of Hepatology, Nork Clinical Hospital of Infectious Disease, Yerevan, Armenia

17 Department of Gastroenterology and Hepatology, Sir Ganga Ram Hospital and GRIPMER, New Delhi, Delhi, India

18 Department of Medicine, 302 Millitary Hospital, Beijing, China

19 Department of Gastroenterology and Hepatology Sciences, IMS \& SUM Hospital, Bhubaneswar, Odisha, India

20 Asian Institute of Gastroenterology, Hyderabad, India
21 Division of Gastroenterology and Hepatology, Department of Medicine, National University Health System, Singapore, Singapore

22 Digestive Disease and GI Oncology Centre, Medistra Hospital, Jakarta, Indonesia

23 Department of Hepatology, Global Hospitals, Mumbai, India

24 Department of Gastroenterology, VGM Hospital, Coimbatore, India

25 Fatima University Medical Center Manila, Manila, Philippines

26 Department of Medicine, Ziauddin University Hospital, Karachi, Pakistan

27 Department of Medicine, Ankara University School of Medicine, Ankara, Turkey

28 Department of Medicine, University of Santo Tomas, Manila, Philippines

29 Department of Hepatology, Foundation Nepal Sitapaila Height, Kathmandu, Nepal

30 Department of Medicine, Humanity and Health Medical Group, New Kowloon, Hong Kong, China

31 Department of Hepatology, Sir Salimullah Medical College, Dhaka, Bangladesh

32 Egyptian Liver Research Institute And Hospital, Cairo, Egypt

33 Division of Hepatobiliary, Department of Internal Medicine, Faculty of Medicine, Cipto Mangunkusumo Hospital, Universitas Indonesia, Jakarta, Indonesia

34 Department of Medicine, Queen Mary Hospital Hong Kong, The University of Hong Kong, Hong Kong, China

35 Department of Pediatric Hepatology, Institute of Liver and Biliary Sciences, New Delhi, Delhi, India

36 Department of Hepatobilliary Pancreatic Surgery and Liver Transplant, Institute of Liver and Biliary Sciences, New Delhi, Delhi, India

37 Professor Emeritus, Chiba University, Chiba, Japan

38 Department of Medicine, University of Kelaniya, Ragama, Sri Lanka

39 Department of Gastroenterology, Ren Ji Hospital, School of Medicine, Shanghai Jiao Tong University, Shanghai, China

40 CHESS Frontier Center, The First Hospital of Lanzhou University, Lanzhou University, Lanzhou, China 
41 Department of Medicine, Tokyo University School of Medicine, Tokyo, Japan

42 Department of Gastroenterology and Hepatology, Faculty of Medicine, Saitama Medical University, Saitama, Japan

43 New Zealand Liver Transplant Unit, Auckland Hospital, Auckland, New Zealand

44 Yangon GI and Liver Centre, Pabedan, Yangon, Myanmar

45 Division of Hepatology, Department of Gastroenterology and Hepatology, Chang Gung Medical Foundation, Linkou Chang Gung Memorial Hospital, Taoyuan, Taiwan

46 Department of Liver Transplant Surgery, Dr. Rela Institute and Medical Centre, Chennai, India

47 Department of Microbiology, Institute of Liver and Biliary Sciences, New Delhi, Delhi, India

48 Department of Pathology, Institute of Liver and Biliary Sciences, New Delhi, Delhi, India

49 Department of Immunohematology and Transfusion Medicine, Institute of Liver and Biliary Sciences, New Delhi, Delhi, India

50 Department of Gatroenterology, SMS Med College, Jaipur, India

51 Division of Liver Transplantation and Hepatology, Manipal Hospitals, Bangalore, India

52 The Liver Unit, Cochin Gastroenterology Group, Ernakulam Medical Centre, Kochi, India

53 Department of Pediatric Gastroenterology, SGPGIMS, Lucknow, India

54 Noora Hospital in Srinagar, Shrinagar, India

55 Department of Gastroenterology and Pediatric Gastroenterology, PGIMER, Chandigarh, India

56 Department of Gastroenterology and Human Nutrition, AIIMS, New Delhi, India

57 Department of Gastroenterology, Hepatology and Liver Transplant, B L K Hospital, New Delhi, India
58 Centre for Liver and Biliary Science, Max Hospital, New Delhi, India

59 Department of Gastroenterology, Hepatology and Liver Transplant, Max Hospital, New Delhi, India

60 Department of Pathology, GB Pant Hospital, New Delhi, India

61 Department of Liver Transplant and Hepatobilliary Surgery, Fortis Hospital, New Delhi, India

62 Department of Gastroenterology, GB Pant Hospital, New Delhi, India

63 Department of Gastroenterology, Hepatology and Liver Transplant, Apollo Hospital, New Delhi, India

64 Department of Hepatology, Sir H N Reliance Hospital and Research Centre, Mumbai, India

65 Department of Gastroenterology and Hepatology, Narayana Hospital, Gurugram, India

66 Department of Hepatology, Parimal Multi-Speciality Hospital, Ahmedabad, India

67 Department of Nephrology, Institute of Liver and Biliary Sciences, New Delhi, India

68 Department of Statistics and Clinical Research, Institute of Liver and Biliary Sciences, New Delhi, India

69 Department of Hepatology and Gastroenterology, Kalinga Institute of Med Sciences, KIIT University, Bhubaneswar, India

70 Department of Hepatology, Institute of Post Graduate Medical Education and Research, Kolkata, India

71 Department of Internal Medicine, College of Medicine, The Catholic University of Korea, Seoul, South Korea

72 Department Of Internal Medicine, Inje University College of Medicine, Busan, South Korea 